University of Louisville ThinkIR: The University of Louisville's Institutional Repository

Electronic Theses and Dissertations

$5-2018$

\title{
A case study of teacher and principal perceptions of a school-wide positive behavior intervention and supports framework in an urban elementary school.
}

Cassaundra Lynn Watkins

University of Louisville

Follow this and additional works at: https://ir.library.louisville.edu/etd

Part of the Elementary Education Commons

\section{Recommended Citation}

Watkins, Cassaundra Lynn, "A case study of teacher and principal perceptions of a school-wide positive behavior intervention and supports framework in an urban elementary school." (2018). Electronic Theses and Dissertations. Paper 2908.

https://doi.org/10.18297/etd/2908

This Doctoral Dissertation is brought to you for free and open access by ThinkIR: The University of Louisville's Institutional Repository. It has been accepted for inclusion in Electronic Theses and Dissertations by an authorized administrator of ThinkIR: The University of Louisville's Institutional Repository. This title appears here courtesy of the author, who has retained all other copyrights. For more information, please contact thinkir@louisville.edu. 
A CASE STUDY OF TEACHER AND PRINCIPAL PERCEPTIONS OF A SCHOOL-WIDE POSITIVE BEHAVIOR INTERVENTION AND SUPPORTS FRAMEWORK IN AN URBAN ELEMENTARY SCHOOL

By

Cassaundra Lynn Watkins

A.A., University of Louisville, 1992

B.S., University of Louisville, 1999

M.A.T., University of Louisville, 2007

Ed.S., University of Louisville, 2012

A Dissertation

Submitted to the Faculty of the

College of Education and Human Development of the University of

Louisville in Partial Fulfillment of the Requirements for the Degree of

Doctor of Education

in Educational Leadership and Organizational Development

Educational Leadership, Evaluation, and Organizational Development

University of Louisville

Louisville, Kentucky

May 2018 

A CASE STUDY OF TEACHER AND PRINCIPAL PERCEPTIONS OF A SCHOOLWIDE POSITIVE BEHAVIOR INTERVENTION AND SUPPORTS FRAMEWORK IN AN URBAN ELEMENTARY SCHOOL

By

Cassaundra Lynn Watkins

A.A., University of Louisville, 1992

B.S., University of Louisville, 1999

M.A.T., University of Louisville, 2007

Ed.S., University of Louisville, 2012

A Dissertation Submitted on

May 8, 2018

to the following Dissertation Committee:

Meera Alagaraja Co-Chair

Blake Haselton Co-Chair

William Ingle

Deborah Powers 


\section{DEDICATION}

First and foremost, I thank God for giving me the strength to persevere and finish this marathon of a six-year endeavor. God makes all things possible and provided me with the support of my husband and children who unselfishly motivated me to achieve my goal. Along the way, my desire to continue sometimes wavered and I constantly looked to them for support, empathy, and ultimately, the strong push I needed to remain committed and keep my eyes focused on the prize. They were ALWAYS there for me and for that I am exceedingly grateful and blessed. My family loves me unconditionally, constantly tells me they are proud of me and I dedicate this work to them. I undertook this journey to grow as an educator and a researcher, but more importantly to be a part of the changes needed to take place in education in the future for my own children and all the children who attend public schools in the United States.

Jerry, Jordan, Ryan, Devin and Michaela...I love you with all my heart. 


\section{ACKNOWLEDGEMENTS}

I would like to thank Dr. Alagaraja for her guidance and support to help me complete this challenging endeavor. I would also like to thank the other committee members, Dr. Kyle Ingle, Dr. Blake Haselton and Dr. Deborah Powers for their mentorship, feedback and assistance in ensuring the successful completion of my doctoral coursework and dissertation journey. 


\begin{abstract}
A CASE STUDY OF TEACHER AND PRINCIPAL PERCEPTIONS OF A SCHOOLWIDE POSITIVE BEHAVIOR INTERVENTION AND SUPPORTS FRAMEWORK IN AN URBAN ELEMENTARY SCHOOL

Cassaundra Lynn Watkins

May 8, 2018

This single site case study examined the perceptions of teachers and the principal of a persistently low achieving elementary school in a large urban school district. Using the Concern-Based-Adoption-Model (CBAM), which originated in Fuller's (1969) study of perceptions of pre-service and practicing teachers and used by Roach, Kratochwill and Frank (2009) as a tool to ascertain support for the implementation of research-based practices, teacher and principal perceptions of the effectiveness of School-Wide Positive Behavior Interventions and Supports (SWPBIS) framework were examined. Interviews and document analysis were utilized to collect data and triangulate teacher and principal perceptions of obstacles and supports to implementation, sustainability and overall effectiveness of SWPBIS to lessen disruptive and unsafe student behaviors and improve the culture and climate of the school for students and staff. Conclusions and implications for practice and future research were also discussed.
\end{abstract}


TABLE OF CONTENTS

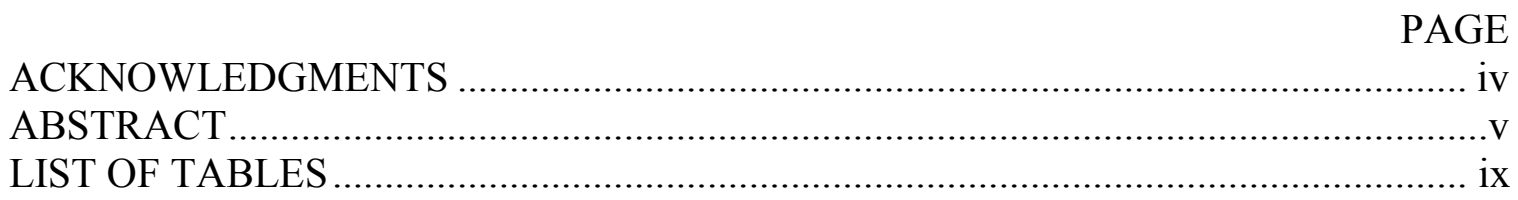

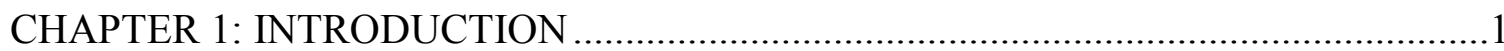

Background of the Problem .......................................................................................

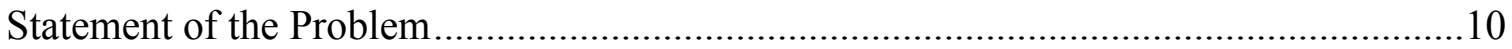

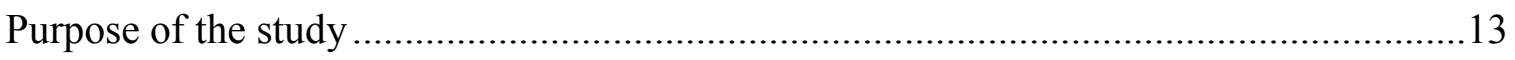

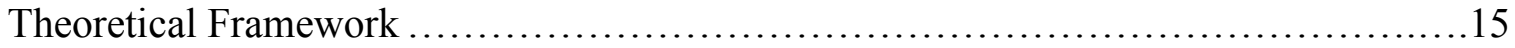

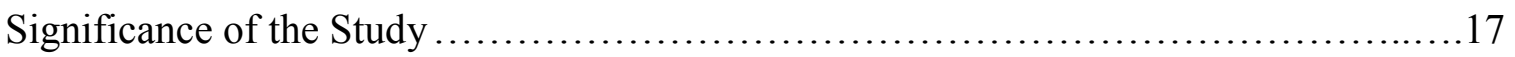

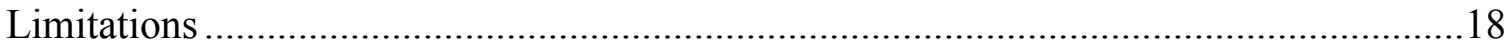

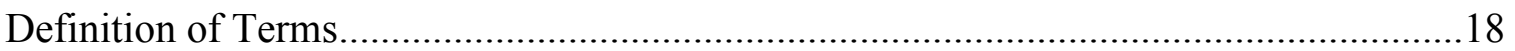

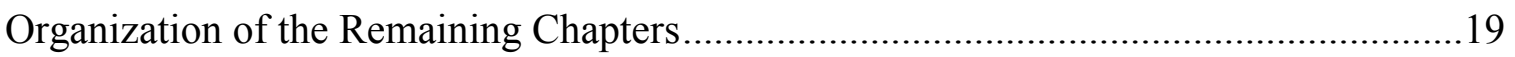

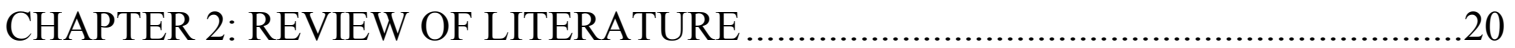

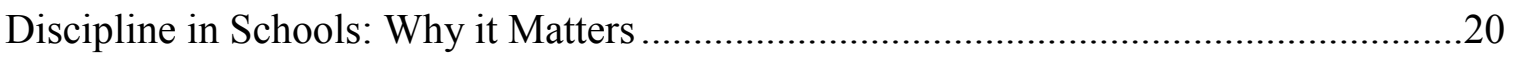

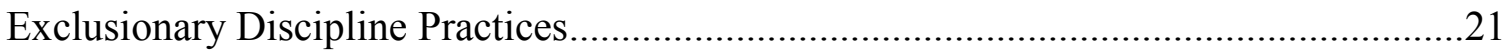

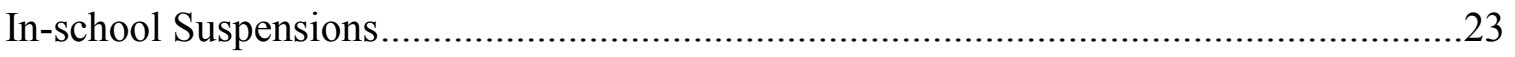

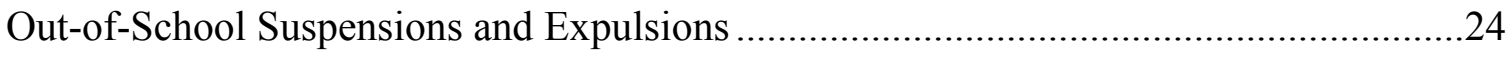

Factors Associated with Exclusionary Disciplinary Decisions ……………………........26

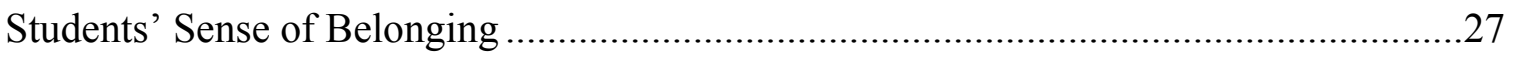

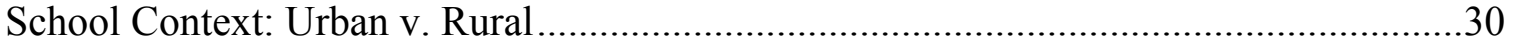


Student Demographics

School Climate and Exclusionary Discipline Decisions...............................................34

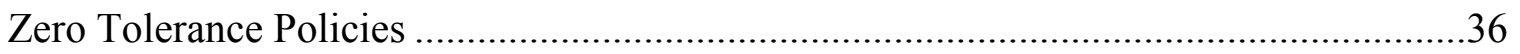

Policies and Practices to Mitigate Exclusionary Discipline Practices ............................38

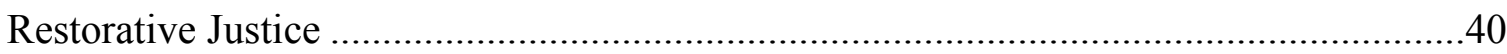

Positive Behavior Interventions and Supports .................................................. 40

Positive Behavior Interventions and Supports Implementation......................................43

Positive Behavior Interventions and Supports Effectiveness ......................................47

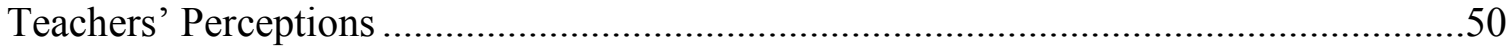

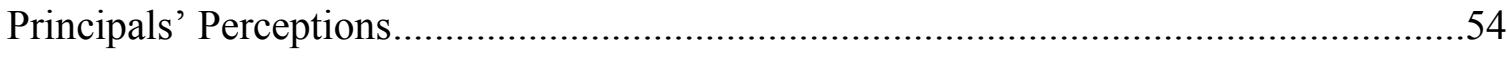

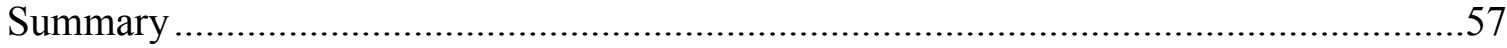

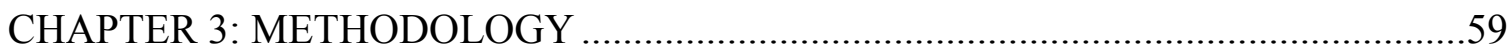

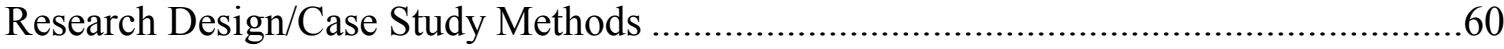

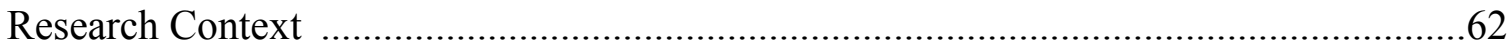

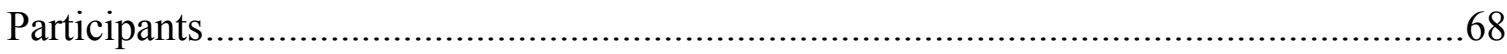

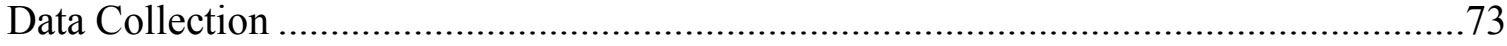

Interview Protocol .............................................................................................. 76

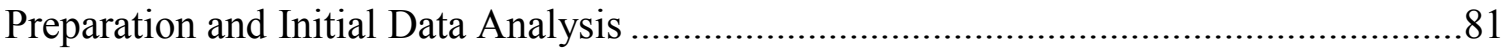

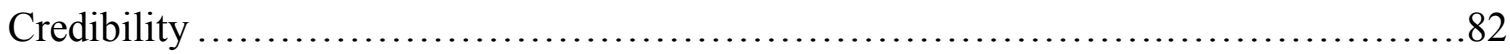

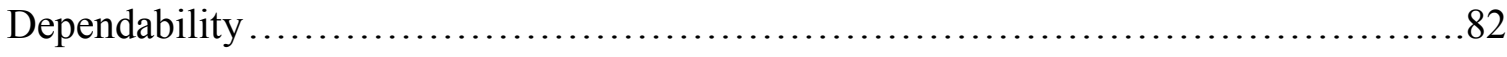

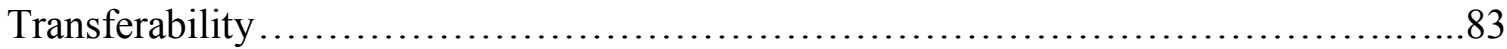




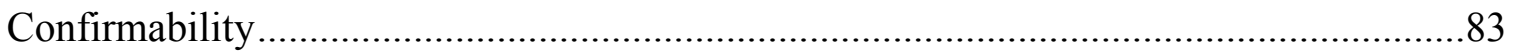

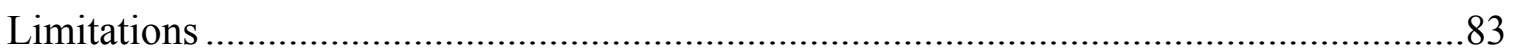

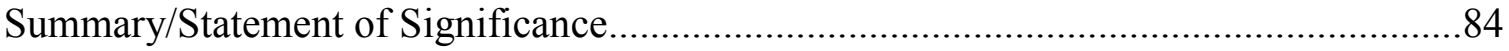

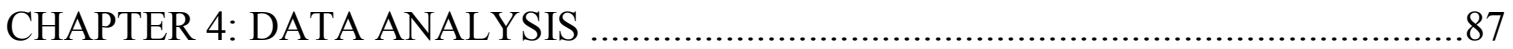

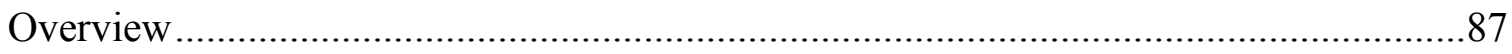

Participant Perceptions of Positive \& Negative Behaviors.................................................88

Participant Perceptions of Overall SWPBIS Effectiveness ...........................................108

Participant Perceptions of Obstacles and Supports to SWPBIS …………………….......123

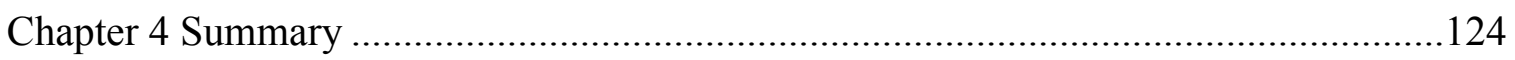

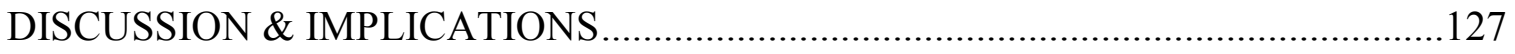

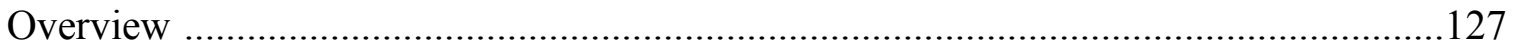

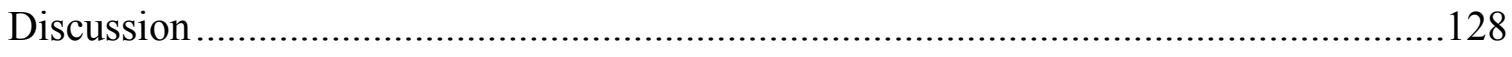

Implications \& Recommendations............................................................................134

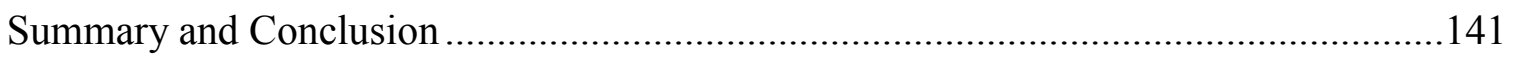

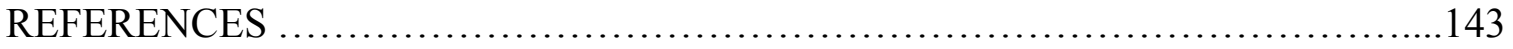

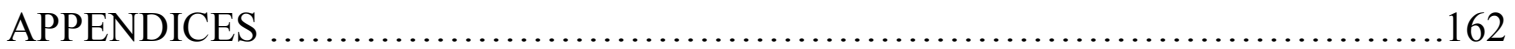

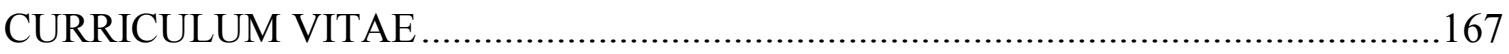




\section{LIST OF TABLES}

TABLE PAGE

1. Summary of Participant Perceptions of Positive and Negative Student Behaviors.......89

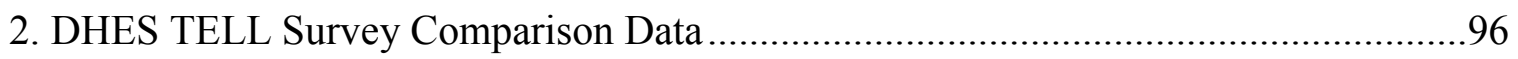

3. DHES School Discipline Referral Trend Data ………..................................................103

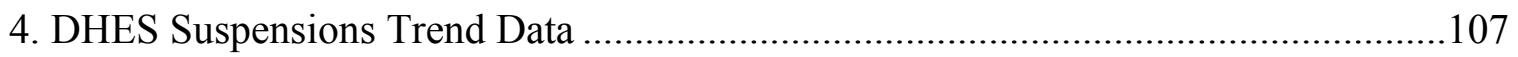




\section{CHAPTER I}

\section{INTRODUCTION}

According to Leone, Mayer, Malmgren, \& Meisel (2000):

Most parents and members of communities believe that schools should be places where children develop intellectually and socially. The idea that school violence, in whatever form, interferes with the orderly operation and safety of schools is anathema to the public. Beyond concerns about physical injury to children, disruption of the school environment interferes with others' learning and can create a climate of fear in which children avoid school or engage in behaviors to protect themselves. (p. 1).

School violence in America has emerged as a "significant public health crises warranting immediate attention" (McMahon et al., 2014, p. 753). In 2010, nationally, 11 percent of high school students reported being in a fight, 6 percent reported carrying a weapon on school grounds within the past thirty days and 8 percent reported being threatened or injured by a weapon on school property (NCES, 2010). Violence in schools negatively impacts student academic achievement and behavior, adversely affects teacher and staff job performance and impairs teacher and parent relationships (Daniels, Bradley \& Hays, 2007). School violence and discipline problems have permeated all aspects of public education: rural, suburban and urban, but urban, public schools may have the unfortunate distinction of possessing an unequal allotment of student behavioral, discipline and safety issues. 
The complex dilemma of student discipline, or lack thereof, in urban public schools is a national issue across the United States and has created a sense of urgency for educators to respond (McMahon et al., 2014; Skiba \& Peterson, 2000). Discipline issues found in urban public schools exist in all grade levels: elementary, middle and high school. A 2004 report from the National Center for Education Statistics (NCES), found that urban middle schools were likely to report racial tensions, verbal abuse of teachers, bullying and wide-spread disorder in classrooms. The same report found urban high schools were more likely to report the existence of gang or extremist activity and, as the size of the school population increased, so did the likelihood of discipline problems (NCES, 2004).

Sadly, school safety and student discipline issues are not limited to students having difficulty getting along with other students and following school rules. Working with youth in urban settings presents a superabundance of unique challenges for teachers as well. Today's teachers require more perspective and savvy to proficiently educate more diverse populations of students, including higher percentages of students who possess significant learning deficiencies and/or behavioral problems, mental health needs, limited English proficiency, and students whose families deal with insurmountable financial barriers (Sugai, et al., 2000). Linking teacher-directed violence and work performance in the U.S., one study found $17 \%$ of new teachers leave the profession within in the first five years of teaching (Gray \& Taie, 2015).

Further, McMahon et al. (2014) argue specific teacher and community characteristics are associated with an increased likelihood of victimization, namely, male teachers and urban settings. Recent data from a 2013 report from the National Center for 
Education Statistics (NCES) indicates school related incidents of theft and violence including violence against teachers are on the rise (2014). In addition, the same NCES report states the number of teachers who report students have physically attacked them is at the highest on record.

Student misbehavior in schools is often the daily lead story on television and cable news, internet, and print newspaper reports about students not following rules at school, on the bus and on school grounds. The problem of school violence and student misbehavior has heightened such that, in some cities in the United States, parents are suing schools in federal court alleging negligence to provide a safe environment for students and teachers.

In 2016, a group of parents and families filed a class action lawsuit against the New York City Education Department and its chancellor alleging school violence is increasing, underreported and disproportionately impacts black, Hispanic, gay, bisexual and/or transgender students. In addition, the lawsuit alleges the atmosphere at New York City Public Schools deprives students of their right to receive an education free of harassment, bullying and violence (Harris, 2016). The filing of this lawsuit proves parents are becoming increasingly aware of the increased incidences of violence in public schools occurring because of increased student misbehavior and violence.

As previously stated, violence in public schools is not limited to students. Teachers are increasingly becoming the recipients of assaults by their own students. A national report from the 2007-2008 school year found approximately 18 percent of city schools reported student acts of disrespect toward teachers (excluding verbal abuse) happen daily or at least once per week, a higher percentage than that reported by 
suburban schools (9 percent), town (11 percent), or rural schools (5 percent) (NCES, 2009). More recently, a 2016 report from the Kentucky Center for School Safety (KCSS) found third degree assaults of certified and classified staff by students rose by more than $50 \%$ in the 2014-2015 school year (KCSS, 2016). The 2014-2015 School Safety Data Report on Law reported 5,565 students committed 6,206 law violations and state these data are troubling because the assaults include bus drivers, custodians and school support staff. Unfortunately, these unsettling reports are not, by any means, a new phenomenon.

In the early1990s, many U.S. schools responded to this student discipline crises by replacing their behavior systems, which previously included graduated sanctions for misbehavior, with zero tolerance policies and hiring school resource officers in schools. This change resulted in a disproportionate increase in suspensions of African American boys and students with emotional/behavioral disorders (Gregory, 1997; Leone, Mayer, Malmgren \& Meisel, 2000; Skiba, 2001).

Throughout the 1990s, researchers and the public agreed that school violence and student behavior problems were waxing out of control. Noguera (1995) reported student behavior and violence in public schools had reached crises proportions and extreme changes were necessary to ensure the future of public education in the United States. Further, Noguera stated the problem of school violence was indicative of the overall increase in violence in society and had become one of the most important educational issues in the United States at that time. At the end of the same decade, a survey reported the public rated fighting/gangs/violence, lack of discipline, lack of funding and the use of drugs as the top four problems facing schools and, not surprisingly, these have been the 
top four problems, though not in the same rank order, for over fifteen years (Gallup, Elam \& Rose, 1998).

Classroom disruptions are commonplace in urban, public schools and there is significant nationwide controversy regarding the disproportionate amount of suspensions between white students and students of color (Noguera, 1995). In some cases, student misbehavior consists of internalized behaviors such as social withdrawal and depression while externalized problem behaviors include destructive, aggressive and antisocial behaviors which negatively impact families, schools and entire communities (Sugai et al., 2000). In short, teachers and school administrators nationwide are increasingly less able to manage a progressively wide variety of student misbehaviors and this challenge has caused school districts to rethink their current approaches to managing school discipline and consider new approaches.

The context of urban schools includes higher rates of community poverty, higher rates of teacher turnover and limited fiscal resources and, nationally, test scores and achievement gaps in math and reading are more pronounced in urban schools when compared to suburban and rural schools (Council of the Great City Schools, 2013). In addition, children living in poor, urban environments undergo daily exposure to dilapidated neighborhoods, homes, businesses and public buildings which puts them at risk for environmental risk factors and danger from criminals, drug dealers and prostitutes who use these neighborhoods to hide from law enforcement (Dishion, French \& Patterson, 1995; Rutter, 1981).

Students attending urban schools have higher rates of absenteeism, are more likely to engage in risk taking behaviors and require more intentional teacher discipline 
strategies than students in suburban or rural schools (Lippman, McArthur \& Burns, 1996). Additionally, a 2009 NCES report from the 2007-2008 school year, found about 13 percent of city schools reported at least one gang crime, which is higher than suburban schools ( 5 percent), towns ( 5 percent) and rural schools ( 3 percent).

The staggering inequalities in wealth and income in America as a whole fully exist and are practically mirrored in its education system. A recent research study analyzed changes in wealth holdings between black and white families following the recession in 2009 and found wealth inequality reached its 25 -year high in 2009 with the median white family holding 22 times more wealth than the median black family (Fox, 2015). The United States has the most unequal distribution of wealth and income of any industrialized country and students living in high poverty areas and high minority schools routinely have fewer resources to ensure access to an equitable learning environment (Dolph, 2017; Smeeding, 2001).

Children living in high poverty areas and attending urban schools are at risk of exhibiting antisocial behavior due in part to exposure to significant family and community-based risk factors (McCurdy, Mannella \& Eldridge, 2003). These risk factors include: parents and older siblings who may already have experienced negative encounters with police, sell or are addicted to drugs and/or alcohol and expose the child to abusive situations including physical and sexual abuse. Thus, students who begin their schooling at high poverty urban schools often bring with them the baggage of stress and trauma that results from their environment and are not prepared for learning in a public school system that is ill-equipped to accommodate their specific needs. Based upon these situational conditions and other high-risk factors, students from high poverty, urban areas 
tend to engage in more antisocial behaviors as compared to students not living in high poverty, urban areas.

Despite the changing needs and demographics of America's students, public schooling in the United States has remained virtually unchanged. Noguera (2003) states that in American society, public schools exist to fulfill a social purpose consisting of three primary functions. First, schools sort children based upon their academic ability and place them on tracks that ultimately determine their occupations as adults. This intentional sorting of roles influences which students manage corporations and government and which students are managed by those in charge (Bowles \& Gintis, 1976: Oakes, 1985). Second, schools play a monumental role in the socialization of children by imbuing them with the values and norms acceptable to the social order, such as respecting rules and authority. Additionally, schools teach students a sense of what it means to be "normal" in a civil society (Gottfredson, 2001). Lastly, public schools function as institutions of social control by providing custodial care and movement of students.

Casella (2001) states that schools operate as surrogate parents and exercise substantial authority over students to the point that many of their basic civil rights are suspended while in school. After being in this tracking system for a while, students learn whether they are on the path that leads to success and economic security or the path to a dead-end job, low wages and a life of struggling to survive. Those students selected for the successful track willingly accept their placements believing they deserve to attend college and be prosperous in their careers. 
In contrast, students placed on the vocational or non-college track tend to be the students that cause the behavior problems and classroom disruptions in school. This is because they have figured out the rewards of education are not available to them and therefore do not value the rules and expectations of a system that does not value them or their abilities (Noguera, 2003). Further, Noguera asks, "should we be surprised that they become more likely to disrupt the educational process?” (p. 345).

Student discipline problems do not appear magically in middle and high school. Elementary students in high poverty, urban communities arrive at school with increased levels of aggressive behavior. Guerra, Huesmann, Tolan, Van Acker and Eron (1995) conducted a study on aggressive behavior in young children from low income, urban areas and found the percentage of students demonstrating clinical levels of aggression more than doubled from first to second grade. Moreover, findings suggest the percentage was more than four times greater than the national average by the end of fifth grade.

U.S. schools began criminalizing student misbehaviors such as rule-breaking and dis-obedience resulting in comparisons of schools, more specifically urban schools, to prisons (Hirschfield, 2008). In addition, Devine (1996) found schools facing substantial problems of gangs and violence are more likely to treat students like criminals through enforcement of crime control and management of student misbehaviors. Schools consisting of large populations of disadvantaged urban minorities criminalized student misbehaviors at a much higher rate that schools without large populations of poor students of color (Wacquant, 2001).

As administrators continually funneled students, most likely students of color, into the criminal justice system for infractions that previously called for detention and 
suspensions, the school to prison pipeline was constructed. The phrase "school-to-prison pipeline" refers to a journey through school consisting of repeated suspensions, identification as a special education student, retention, alternative school placements and ultimately dropping out or getting pushed out of school altogether (Wald \& Losen, 2003).

School Districts began to receive major criticism from parent and advocacy groups questioning the legality of their zero tolerance policies (Advancement Project \& the Civil Rights Project, 2000). The criminalization of student misbehavior caused a disproportionate impact on students of color all while teachers and administrators routinely labeled students as future prisoners and justified their coercive control and exclusion policies (Skiba et al., 2003). In response to this criticism, as early as 2001-2002 school year, many school districts in the U.S. began implementing School-Wide Positive Behavior Interventions and Supports (SWPBS), commonly referred to as Positive Behavior Supports (PBS) and Positive Behavior Interventions and Supports or PBIS (SWPBS; Sugai \& Horner, 2006).

Positive Behavior Supports initially developed as a behavioral intervention approach used for students with significant disabilities who engaged in extreme aggression and self-injury (Durand \& Carr, 1985: Meyer \& Evans, 1989). School-Wide Positive Behavior Interventions and Supports (SWPBIS) focuses on improving school climate, student achievement and social behavior and is associated with the reduction of teacher stress due to challenging school climates and pressure to produce higher student outcomes, i.e., test scores (Ross, Romer \& Horner, 2011).

In 2001, the Kentucky Instructional Discipline and Support (K.I.D.S.) Project was created by the Commonwealth of Kentucky elected to create a more positive, safe 
and supporting learning environment for students and staff (Kentucky Center for Instructional Discipline, 2015). The Kentucky Department of Education has expanded this program statewide via the Kentucky Center for Instructional Discipline (KCID), which is part of the Kentucky PBIS Network. KCID defines Positive Behavior Interventions and Supports (PBIS) as a multi-tiered systems framework focused on establishing a positive learning environment where all students are set up for success.

PBIS focuses on the teaching and re-teaching appropriate behaviors in a preventative approach to student discipline while utilizing pre-planned interventions for students who required additional behavior supports. Schools receive training through the Kentucky Center for Instructional Discipline over the course of a year to prepare them for the first year of PBIS implementation which includes: analysis of school behavioral data, creation of school-wide behavioral expectations and the development of a plan to ensure all staff members are trained efficiently prior to implementation of PBIS.

\section{Statement of the Problem}

Student discipline and classroom management challenges plague urban schools (McKinney, Campbell-Whatley, \& Kea, 2005). Skiba et al (2014) found teachers in high poverty, urban schools spend more time dealing with student behavior and discipline problems such as disruptive behavior, fighting and use of possession of weapons or drugs. During the 2013-2014 school year, 1.3 million discipline incidents were reported in the United States for reasons related to drugs, alcohol, violence or weapons possession resulting in a student being removed from the education setting for at least one day (NCES, 2015). 
Apparently, this disturbing trend still proves to be problematic as a 2015 report for the National Center for Education Statistics found in 2014, among students ages 1218 , about 850,000 nonfatal victimizations occurred at school including theft, simple assault and serious violent victimizations (NCES, 2015). Policies to promote equitable school environments for all students have been the focus of the federal government since it enacted the Elementary and Secondary Education Act (ESEA) in 1965 (Thomas \& Brady, 2005).

ESEA included several major educational reform objectives including greater academic accountability for students, increased local control, improved teaching methods, and expanded options for parents, which are still in existence today. In 2001, President George W. Bush reauthorized and renamed ESEA as the No Child Left Behind (NCLB) Act which required all states to establish state academic standards and a state testing system in accordance with federal standards. (Thomas \& Brady, 2005). In 2015, the U.S. congress passed the Every Student Succeeds Act (ESSA), which reauthorizes No Child Left Behind and continues to require states to provide interventions for schools scoring in the lowest five percent of schools in the state on state created annual assessments.

Under NCLB guidelines, these schools were identified as Persistently Low Achieving (PLA) schools due to having an overall score in the lowest 5\% of all Title I or Title I-eligible schools failing to make the annual measurable objective (AMO) for the last three consecutive years, as measured by the annual state accountability tests. These PLA schools serve students who are persistently underachieving and targeted for additional interventions and support from the state department of education. 
For the purposes of this study, persistently low achieving (PLA) and low performing schools are synonymous as identified by NCLB, ESEA and ESSA and as addressed in education research and literature. The current federal statute and final regulations of ESSA specify that states must identify certain schools at least once every three years for comprehensive support and improvement. Collectively, low performing schools tend to have increased instances of behavior issues, low-performing students, high rates of poverty and percentages of minority students and increased levels of teacher stress and burnout. This research study examines elementary teacher and principal perceptions on the overall effectiveness of SWPBIS, under the additional stresses of PLA school status (low-performing students), in an urban, public school district in the Southeast Region of the United States.

Over time, the pendulum has swung, with regard to managing student behavior problems, from the extremely punitive zero tolerance policies to the more relaxed nonpunitive, positive intervention framework of PBIS. Despite research supporting the use of PBIS as a method to decrease behavioral issues and violence in public schools (Sugai \& Horner, 2008), since the adoption of Positive Behavior Interventions and Supports, schools in this school district are showing constant and increased rates of student behavioral infractions (NCES, 2015). The numbers of behavioral instances reported in this school district selected for this study is extremely high and should raise concerns as to whether PBIS is effectively decreasing the number of behavior problems in schools. Researchers suggest the implementation of position behavioral practices will decrease the incidences of student misbehaviors in school and increase the overall perception of student misbehavior in a school (Bohanon et al., 2006). 


\section{Purpose of the Study}

This study seeks to listen to the voice of teachers and administrators to determine their overall perceptions of whether PBIS works or does not work to reduce student misbehavior, suspensions and improve the overall classroom and school climate in two elementary schools located in a large, urban school district in the Southeast Region of the United States. This study seeks to analyze what cannot be determined through statistics and data reports. Behavior data are easily available from the state department of education and the website of the school district used in this study. The data can be analyzed to draw conclusions about PBIS effectiveness to reduce student behavioral issues and improve overall school climate.

In the PBIS framework, the classroom teacher is the first line of defense in providing corrective counseling and options to decrease the negative behaviors and encourage the student make positive choices that will lead to student academic and nonacademic success in school. Teacher's perceptions of student misbehavior have been cited as a leading cause for teachers exiting the teaching profession (Barnes, Crowe, \& Schaefer, 2007). A report from the National Center for Education Statistics (NCES) found that student behavior issues were worse in urban, public schools than in suburban or rural schools and teachers spend more time maintaining classroom discipline in urban schools (NCES, 1996).

The urban, public school teachers are the soldiers on the front line charged with the implementation of SWPBIS in urban, public schools and it is imperative that they weigh in on the effectiveness of the PBIS framework. Warren et al. (2003) found the unique challenges of urban schools require more preparation and training before PBIS 
can be successfully implemented. Some research has been done on PBIS effectiveness in suburban and rural schools, few studies have focused on the effectiveness of PBIS in large, urban, public schools where there is an increased mix of ethnic and culturally diverse students, high rates of students living in poverty and a greater predominance of antisocial behavior school-wide.

Research on teacher voice and PBIS is necessary because previous qualitative research studies on the effectiveness of PBIS focused on teacher ratings of the impacts of aggressive student behavioral issues, concentrations of problem behaviors, office discipline referral use and impact on suspensions (Bradshaw, Mitchell \& Leaf, 2010).

The focus of this research study is to ascertain teacher and principal perceptions of the overall effectiveness of School-Wide Positive Behavior Intervention Supports (SWPBIS) in decreasing student misbehaviors and suspensions in a low-performing elementary school in a large, urban public school district in the Southeast Region of the United States. Interviews will be used to capture the teacher and principal perceptions of SWPBIS effectiveness in the participants' own words so their invaluable voices may be heard and contribute to possible changes in future SWPBIS policies and procedures initiated by the school district used in this study. My research questions are rooted in a pragmatic framework focusing on the application of PBIS in an effort to identify solutions to improve to the current school discipline problems in schools.

\section{Research Questions}

In this study, I seek to answer the following research questions:

- How do teacher and principal perceptions of School-Wide Positive Behavior Interventions and Supports impact its effectiveness? 
- What do teachers and principals in urban, low-performing schools perceive as obstacles and supports to SWPBIS implementation, sustainability and effectiveness?

\section{Theoretical Framework}

Pragmatism entails dealing with problems in a practical way rather than using theories or abstract theories in terms of determining their success or truth. Pragmatic researchers are concerned with the "what" and the "how" of research and the intended consequences, which in this case would be improved student behavior resulting in improved school culture, teacher well-being and overall student achievement (Creswell, 2013, p. 28). Pragmatic research focuses on the anticipated consequences that the research may bring to light (Cherryholmes, 1992). Recall the purpose of this study is to determine factors contributing to the successful or unsuccessful school-wide effectiveness of Positive Behavior Interventions and Supports (PBIS) in urban, low performing schools in a public school district in the Southeast Region of the United States.

Edmonds (1982) theorized five correlates that form the basis of an effective school:

1. Principal leadership with a focus on substantial attention to the quality of instruction

2. Clarity of instructional focus

3. Orderly, safe climate conducive to learning

4. Teacher behaviors that show high expectations for all students to obtain minimum mastery 
5. Use of measures of pupil achievement as the basis of program evaluation In a perfect United States, a safe school environment would exist in all public schools. The stark reality is that inappropriate student behaviors such as, aggression, disruption, bullying and violence are rife in our nation's public schools and the situation is even worse in urban public schools (Handler, Rey, Connell, Their, Feinberg \& Putnam, 2007). In addition to creating an unsafe learning environment, managing inappropriate student behaviors and classroom disruptions is time-consuming, interferes with student engagement and wastes valuable instructional time (Riley, McKevitt, Shriver \& Allen, 2011). It is crucial that all U.S. schools, and specifically urban, high poverty schools, move from our current as-is state of heightened behavioral problems to our desired state consisting of a safe, orderly atmosphere conducive to high quality teaching, learning and continuous student achievement.

School-Wide Positive Behavior Interventions and Support (SWPBIS) is defined as a framework that utilizes whole-school social culture and multi-leveled tiers of behavior support intensity to improve social and educational outcomes for all students (Horner \& Sugai 2015). The decision to study SWPBIS effectiveness is the result of my work in the field as an educational recovery leader working in urban, low-performing schools in a large district in the Southeast Region of the United States. In this position, this researcher daily witnesses distressing incidences of out of control student behavior in classrooms, hallways, inside the cafeteria and on the bus loading dock during dismissal each day.

This researcher's primary role entails coaching principals, teachers and working to implement sustainable systems resulting in improved academic achievement to move 
schools out of priority status. For this study, the researcher will conduct a single site case study at an urban, low-performing public school in a school district in the Southeast Region of the United States where there is a disturbing trend of student misbehavior.

The decision to study the effectiveness of SWPBIS stems from a question of what "works" for teachers and administrators dealing with rampant inappropriate student misbehavior daily when there is a district conduct policy in place to manage student behavior (Patton, 1990). This researcher's concern lies with the practical application, or lack thereof, of the SWPBIS behavioral intervention framework and using teacher and administrator voice to identify possible solutions to improve SWPBIS effectiveness in the future.

\section{Significance of the Study}

This qualitative study will focus on teachers, administrators and school district personnel to determine whether classroom climate and student behavioral issues improved as a result of the implementation of school- wide Positive Behavior Interventions and Supports. To ascertain teacher and administrator perceptions and attitudes, interviews will provide rich data and focus on the classroom experiences, i.e., lived experiences, of teachers implementing SWPBIS in urban, low-performing schools in hopes of improving PBIS effectiveness to produce more positive future outcomes. Specific research calling for this study includes Warren et al (2003) which emphasizes the need for more research on PBIS in urban settings citing urban schools need more preparation before successful PBIS implementation.

The study site is a large, urban public school district in the Southeast Region of the United States. In 2014-2015, this district scored in the $57^{\text {th }}$ percentile of all school 
districts in the state prompting the state department of education to issue a classification of "Needs Improvement". The majority of the state's lowest performing schools are located in this school district, thus prompting the need for determining how teacher and principal perceptions of the effectiveness of PBIS can inform central office administrators and district personnel to facilitate strategies aimed at decreasing student antisocial behaviors and improving overall school climates.

\section{Limitations}

The research for this study will include interviews of teachers and principals currently employed in two low-performing public schools in a large public school district in the Southeast Region of the United States. The schools are identified as lowperforming based upon from annual state accountability testing results, in accordance with state accountability requirements required by the Every Student Succeeds Act (ESSA). Teachers employed in average and higher performing schools are excluded from this study thus preventing generalizability of the study results to all schools.

Nevertheless, unlike quantitative studies, this limitation is minimized by the fact that the goal of most qualitative research studies is not to generalize the results, but rather to provide a rich, thick contextualized understanding of an aspect of human experience through intensive study of a particular case or cases. Thus, this limitation should not hinder the findings of this study.

\section{Definition of Terms}

This study will use the following terms defined for complete and correct implementation: 
Positive Behavior Interventions and Supports (PBIS) is a framework for providing universal systems and support for teaching expectations while planning and preparing for individualized instruction for students requiring additional support.

School-wide Positive Behavior Interventions and Supports (SWPBIS) refers to the schoolwide application of the PBIS framework.

Urban Schools are schools located in inner-city environments characterized by higher concentrations of poverty, greater racial and ethnic diversity, larger numbers of immigrants who speak diverse languages and increased rates of student mobility. Persistently low achieving Schools (PLA) are schools identified, under NCLB guidelines, as having an overall score in the lowest $5 \%$ of all Title I or Title I-eligible schools failing to make the annual measurable objective (AMO) for the last three consecutive years, as measured by the annual state accountability tests. These schools serve students who are persistently underachieving and targeted for additional interventions and support from the state department of education.

\section{Organization of the Remaining Chapters}

This chapter presents an introduction into this research study which seeks to acquire teacher perceptions of the effectiveness of the PBIS to decrease student behavioral issues at low-performing schools in a large, urban school district in the Southeast Region of the United States. Chapter 1 provides the background of the study, purpose of the study, significance, limitations and definition of key terms. The literature review is presented in Chapter 2, methodology in Chapter 3, data analysis in Chapter 4, and a discussion and implications in Chapter 5. 


\section{CHAPTER II LITERATURE REVIEW}

\section{Discipline in Schools and Classrooms: Why It Matters}

Most people would probably agree that our nation's schools should be safe havens for high quality teaching and learning without numerous instances of crime and violence negatively impacting students, teachers, administrators and the school itself (Brookmeyer, Fanti \& Henrich, 2006). Excessive incidents of student misbehavior and violence in schools is a major issue of concern for school administrators, teachers, parents, students and the community-at-large in the United States. Local television news and social media outlets such as YouTube, Twitter and Facebook constantly showcase uploaded videos of students fighting with teachers, other students in and out of school, bus fights, bullying, sexual harassment and even illegal sexual acts among minors either in school or on school buses. Students and staff should be safe in classrooms and on school grounds and the goals of school discipline today are synonymous with the two main goals of discipline identified in the early 1990s: to ensure the safety of staff and students and the creation of an environment conducive to optimal learning (Gaustad, 1992).

The word discipline comes from the same Latin root as the word disciple or discipere, which means to teach or comprehend (Skiba \& Peterson, 2000). Skiba and Peterson (2000) argue that children arrive at school developmentally incomplete and require instruction, socialization and correction to change fundamentally egocentric behavior into socially acceptable interpersonal skills that result in successful interactions. 
Similarly, Duke (1990) argues the necessity for effective school discipline practices that discourage misbehaviors, encourage students to engage in positive, responsible behaviors and provide satisfying school experiences for students. Discipline is monumentally important in schools and especially in classrooms because disruptive student behavior negatively impacts the learning environment by interfering with students learning, diverting administrative time and contributing to teacher burnout (Osher, Bear, Sprague \& Doyle, 2010).

In a 1998 National Center for Educational Statistics report, Violence and Discipline Problems in U.S. Public Schools: 1996-97, researchers have identified a relationship between day-to-day school disciplinary disruptions and increasing levels of serious violence (Skiba, 2000). Discipline problems have continued to rise since the 1990s and a recent report from the National Center for Education Statistics, Indicators of School Crime and Safety:2015, indicates in 2014, there were about 850,000 nonfatal victimizations and 486,400 violent victimizations (simple assault and serious violent victimizations) in U.S. schools (Zhang, Musu-Gillette \& Oudekerk, 2016). The sharp rise in student disciplinary issues and school violence created a sense of national urgency to fix school behavior and ultimately led to the implementation of exclusionary discipline practices.

\section{Exclusionary Discipline Practices}

Exclusionary discipline practices began to appear in schools in the form of chastising, reprimanding or placing a student in the back of the room or out in the hallway for minor offenses and, for more serious infractions-fighting, defiance, cutting class- removal from the class, school or even expulsion (Noguera, 2003). As discipline 
problems continued to rise, schools responded by focusing on controlling student behavior instead of meeting students' academic and social emotional needs. The United States has an unfavorable history of punishing the students who have the greatest academic, social, economic, and emotional needs (Johnson, Boyden, \& Pitz, 2001). Often, students engage in disruptive behavior either due to frustration or embarrassment because they are behind academically and are unable to perform at a level commensurate with grade-level expectations (Hirschi, 1969).

Intentional efforts to control student behavior ushered in disciplinary practices in schools that bore a striking resemblance to the strategies utilized to punish adults in society. Even though negative disciplinary sanctions are the least effective form of social control, our society continues to employ them based upon the idea of achieving social control through continually increasing negative sanctions (Foucault, 1977).

Clark (2004) posited that traditions of power and control are so deeply embedded in our nation's schools and, working in tandem with disciplinary action and student placement decisions, makes a significant addition to the immense over population of African American males in U.S. prisons. When teachers and school administrators perform their jobs in uncaring and disrespectful ways, students blow up or become passive and shut down. They become disengaged, disinterested and drop out - the infamous “3D's" begin, especially for students of color and as early as third grade (Nichols, 2013).

The focus on control and disproportionate punishment of African American males in America's primary and secondary public schools is not a myth fabricated by those who 
desire to play the race card. The data is clear and the following sections discuss data on in-school and out-of-school suspensions in the U.S. in recent years.

\section{In-School Suspensions}

In 1974, the Analyzing Office of Civil Rights data, the Children's Defense Fund, reported suspension rates for African American students two and three times higher than those for white students and elementary, middle and high school levels (Children's Defense Fund, 1974). Recent research finds that students suspended just once in ninth grade $(16 \%)$ face a twofold increase in the probability of dropping out of high school (32\%) (Balfanz, 2015). A 2015 report from the National Center for Education Statistics found in the 2011-2012 school year, 3.4 million public school students in the U.S. received in-school suspensions (NCES, 2016). Removing students from the classroom, in the form of In-School Suspension may be the result of adults having a fixed mindset about students presumed to be out of control and potentially dangerous and such assumptions are the deeply embedded "norm" at many schools (Noguera, 2003).

Often, teachers and administrators think of In-School Suspension as removing the "bad apples" so the rest of the students can continue their learning free from disruptions. Sadly, schools typically remove and punish students who are already behind academically thus further depriving them of opportunities to receive valuable classroom instruction (Noguera, 2003). School discipline is extremely complex and schools deal with a myriad of offenses from students that provoke classroom and school disruptions including: cursing, bullying, defiance, horseplay, rule violation, cutting class, sexual harassment, fighting and vandalism. Students exhibiting these types of behaviors interfere with the learning of others, monopolize administrative time and contribute to teacher 
burnout (Byrne, 1999). Unfortunately, removing these students from the classroom often worsens the student's behavior as segregation or in-school suspension with other antisocial peers can increase antisocial behavior (Dishion \& Dodge, 2005).

According to Ferguson (2001), African American youth, unlike their white counterparts, are disproportionally funneled into "punishing rooms" and "jailhouses" within the school and written off as "bound for jail" and "unsalvageable". Ferguson also notes that prison looms over the heads of African American youth and schools lack the resources necessary to disrupt the inevitable trajectory of these "unsalvageable" students without compromising the teaching and learning provided to those students deserving of high quality education. The result is a self-fulfilling prophecy as researchers continually confirm that students who are repeatedly suspended from school face increased risks of juvenile and adult incarceration (Arum \& Beattie, 1999; Skiba et al., 2003).

\section{Out-of-School Suspensions \& Expulsions}

Consistent with data on in school suspensions, 3.2 million students in the United States received out-of-school suspensions (NCES, 2016). Students identified as engaging in more serious offenses such as assault, fighting, drug sales/ possession or having guns/weapons on school property usually receive more stringent disciplinary measures such as out-of-school suspension and/or expulsion. Unfortunately, research does not support the use of suspension and/or expulsion to improve student behavior and it appears the timing of school suspensions or expulsions matters. Suspensions and expulsions from school are recurrently associated with negative academic outcomes including lower levels of student achievement and higher dropout rates (Christie, Nelson, \& Jolivette, 2004). Research also shows students suspended in elementary or middle 
school have a lower likelihood of graduating from high school on time (Raffaele Mendez, 2003).

Quantitative reports show Black students are two to five times more likely to be suspended than White students (Irvine, 1990). In addition, qualitative findings indicate teachers restrict punitive consequences and reprimands to Black students even though students of other races engage in the same inappropriate behaviors (McCadden, 1998). Data comparisons of cultural interaction styles point out that teachers routinely interpret the behaviors of African American students as inappropriate when the student's actions are unintentionally inappropriate (Hanna, 1988; Weinstein, Curran \& Tomlinson-Clarke, 2004; Weinstein, Tomlinson-Clarke \& Curran, 2003). Teachers are prone to react more severely than required when responding to the inappropriate behavior of Black students and there is evidence that educators might put forth little effort to diminish behavioral concerns prior to their onset when nonpunitive approaches may prove effective (Emihovich, 1983). Once again, this scenario is less likely to occur with White students.

Skiba and Rausch (2004) conducted a study on the relationship between standardized test scores and suspension and expulsion rates for students in the state of Indiana utilizing data from the math and English/Language Arts section of the Indiana State Test of Educational Progress (ISTEP). The researchers controlled for poverty and the percentage of African American student enrollment. Findings indicated schools with high out-of-school suspension rates had fewer students pass the ISTEP test indicating a positive correlation between discipline and achievement and correlations were highest at the secondary level. Despite evidence that suspensions and expulsions fail to improve student behavior and negatively impact student achievement, schools continued to 
employ suspensions and disproportionately target African American students and students living in poverty.

\section{Factors Associated with Exclusionary Discipline Decisions}

The research suggests that exclusionary discipline practices negatively impact students both academically and/or behaviorally. In addition to the negative impacts on student outcomes, researchers have established that exclusionary discipline decisions and practices are not distributed equally among the student population. According to a May 2015 report from the National Center for Education Statistics, Indicators of School Crime and Safety, a higher percentage of students of low socio-economic status (SES) than of students with middle SES had ever been suspended or expelled (29 vs. 17 percent), and both were greater than the percentage of high-SES students who had every been suspended or expelled (9 percent) (NCES, 2016).

A disproportionate number of students from poverty are suspended or expelled at much higher rates than students who come from more affluent backgrounds and this creates a negative impact on future graduation outcomes for these already impoverished students. The same study found the amount of African American students who received out of school suspensions during the 2011-2012 school year (15 percent) was higher than the percentages for students of any other racial or minority group. Asian students only accounted for one percent of out-of-school suspensions than students from any other racial group.

In the 2013-2014 school year, a higher percentage of African American students (36 percent) than of Hispanic (21 percent), White (14 percent), and Asian students (6 percent) had ever been suspended or expelled from school (Zhang, Musu-Gillette \& 
Oudekerk, 2016). Unfortunately, these statistics are nothing new. The overrepresentation of black, male and low-income students is referred to as the discipline gap (Monroe, 2005). In the U.S., African America students are specifically targeted for disciplinary action in higher numbers than any other racial group (Johnston, 2000). The overrepresentation of African American males and females in exclusionary discipline practices such as suspensions and expulsion has been repeatedly affirmed in the literature for more than thirty years (Children's Defense Fund, 1975; Gonzalez \& Szecsy, 2004; Skiba, Michael, Nardo \& Peterson, 2000; Skiba \& Rausch, 2006).

Casella (2003) suggests that the disproportionate representation of African American males is due to school personnel's anxiety and perception that those students who do not fit the social and behavioral norms of the school are inherently "dangerous" or "troublemakers". Teachers who feel threatened or perceive a loss of control in their classrooms tend to escalate issues that could be handled in the classroom resulting in heated exchanges with students thus resulting in the student's removal from the classroom (Bowditch, 1993; Varvus \& Cole, 2002). Teachers who don't create an equitable and welcoming environment for all students preempt more issues with student behavior than would probably not exist if all students felt welcome and valued. This issue deserves discussion and the next topic focuses on the importance of students having a sense of belonging.

\section{Students' Sense of Belonging}

Creating a sense of community is an essential aim of school administrators and teachers to ensure all students feel welcome and a sense of being in the place where they belong. McMillan and Chavis (1986) maintain that community consists of four elements: 
memberships, influence, integration and fulfillment of needs and a shared emotional connection. They further state that in a community, members feel they are a valued member of the group and the group is important to them. Dewey and Vygotsky both considered education to be a social rather than an individual process and Dewey (1958) argued the quality of education is derived from the degree to which individuals form a group.

Abraham Maslow found that a person's need to belong must be fulfilled or satisfied first before any other needs can be met and social acceptance and a sense of belonging are important throughout life (Maslow, 1962). As educators continue to struggle with identifying factors associated with school discipline and the overabundance of African American students receiving in-school and out-of-school suspensions and expulsions, researchers examined how a student's sense of belonging impacts student behavior and discipline in public schools.

Goodenow and Grady (1993) define sense of belonging as the measure of how accepted, respected and included students feel in their school social environment. Educators play a pivotal role in ensuring student's feel welcome and connected to their school environment. Research has found that unless students identify well with their schools, their participation in their own education will be limited (Finn, 1989).

Additionally, a superabundance of factors influence the teacher and student interactions that result in school disciplinary issues and they are strongly impacted by the developmental need of students, student and teacher backgrounds, student socioeconomic status, school and classroom culture and climate, curriculum demands and student and teacher expectations and capacity to meet the expectations expected for their roles 
(Osher, Bear, Sprague \& Doyle, 2010). In short, context matters when considering all the variables that play a part in shaping school discipline decisions and student behavior. Combs (1982) posited that student academic success is contingent upon students having a sense of belonging and caring from school staff.

In a quantitative study of the relationship between students' sense of belonging in school classes and motivation, student effort and academic achievement, 301 multiethnic students from two urban, junior high schools were surveyed to measure the correlation between sense of belonging and measures of motivation and academic achievement. Survey results revealed a student's sense of belonging had a statistically significant impact on motivation, engagement and resiliency to complete difficult academic work (Goodenow, 1991).

Another study surveyed 70 high performing middle school administrators and descriptive analyses found the ability of school administrators to foster students' sense of belonging was paramount for successful school performance (Wendel, Hoke and Joekel, 1994). Further, Edwards (1995) recommended that school administrators must first make sure teachers feel a sense of belonging to the school before they can foster student sense of belonging. Additional research from (Albert, 1990) presented arguments on the importance of students' sense of belonging in three C's: connect, capable and contribute. Albert found teachers need to greet and encourage their students and utilize cooperative learning strategies in the classroom, scaffold students to make them feel capable and assign students classroom duties so they can contribute to the learning community.

A qualitative study of 51 middle school students from two schools in the same district (one urban, one rural) utilized individual and focus-group interviews to examine 
students' perceptions of the characteristics of classroom membership or sense of belonging. Researchers analyzed the data and student responses indicated that having friends in class, interacting with peers, class participation and earning good grades resulted in sense of belonging (Williams \& Downing, 1998). Findings did not indicate a variance in results from urban and rural students.

Finally, sense of belonging is extremely important in students' development of academic resiliency which is crucial to student achievement. Wang, Haertel, \& Walberg (1998) outlined the roles educators play to promote educational resilience despite highrisk factors increasing risk for academic failure. Risk factors include: family poverty, physical illness, divorce, drug abuse, and high mobility rates. Sense of belonging emerged as an integral part of any educational program servicing high risk students to ensure students achieve academic success. This research examining the importance of student's sense of belonging is pertinent to this qualitative case study because it focuses on the role of teachers and principal's working in urban, high poverty schools.

\section{School Context Matters: Rural Schools}

Peterson, Beekley, Speaker \& Pietrzak (1996) examined violence in rural school districts and found $52 \%$ of teachers and administrators perceived that violence was increasing at the secondary school level. However, the types of behaviors they reported were not the same types of deadly violence-gang involvement, weapons, drugs- that concern society most. Violent behaviors reported include: rumors, pushing and shoving by students, verbal and sexual harassment, etc. Apparently, perceptions of school safety are subjective and shaped by the serious violent incidents and overall perceptions of school climate (Skiba \& Peterson, 2000). 
Research from the National Center for Education Statistics in 2014, found that students living in rural areas (53 victimizations per 1,000 students) experienced higher rates of total victimization at school than students living in suburban areas (28 victimizations per 1,000 students) (NCES, 2016). The study found the differences resulted from an increased amount of violent victimization among students living in rural areas. The rate of total victimization at school for students living in urban areas totaled 32 victimizations per 1,000 students. In 2013, a higher percentage of Black and Hispanic students than White students surveyed reported being afraid of an attack or harm both at school and away from school (NCES, 2016). In addition, higher percentages of students living in urban areas than students in suburban areas reported being afraid of being attacked or harmed at school and away from school.

\section{Urban Schools}

In addition to the challenges of poverty, urban, low-performing schools suffer from high rates of disciplinary problems, violent behaviors and suspensions (Handler et al., 2007). Research shows that where children live restricts their access to high quality schools (Cashin, 2014; Orfield, 2002). While all urban public schools are not the same and don't face the exact same challenges, they do share characteristics that differentiate them from suburban and rural schools. According to Kincheloe (2010), urban schools are often characterized by low student achievement, inexperienced teaching staff, poorly functioning data management systems, low expectations of students and a lack of instructional coherence. In addition to these structural challenges, urban schools face additional challenges for teachers and administrators around student discipline and behavior management. 
Discipline problems stifle learning for all students and place higher levels of stress on teachers. Skiba et al (2014) found teachers in high poverty, urban schools are apt to spend more time dealing with discipline issues such as disruptive behavior, fighting and use or possession of drugs or weapons. In addition, Adelman and Taylor (2005) conclude that urban schools face additional challenges such as attendance problems, high dropout rates, physical and sexual abuse and violent behavior. Lastly, Johnson, Marietta, Higgins, Mapp and Grossman (2015) found numerous urban students, in addition to poverty and violence, face racism, have a greater need for special education services and need more support because of the increased numbers of English language learners (ELLs). Thus, student discipline and violence, in addition to the aforementioned problems, are a formidable challenge for urban schools.

\section{Student Demographics}

Research shows that simply being African American in the United States is a major disadvantage for an individual (Hacker, 2010). In 2013, higher percentages of Hispanic (20 percent) and Black (19) percent of students reported the presence of gangs at their school as opposed to White (7 percent) and Asian (9 percent) (NCES, 2016). Thus, school safety becomes an issue and, as previously mentioned, teachers' fear of losing control of their classrooms often leads to unfair and discriminatory discipline practices leveled toward schools with larger enrollments of African American students and students whose families live in poverty. Students considered dangerous, largely poor, African American males, are unfairly targeted and more likely to receive the most punitive discipline consequences (Fenning \& Rose, 2007). Questions arise as to whether 
schools with a majority of African American students are at risk academically and behaviorally.

A quantitative study of public schools in Louisiana sought to determine if there is a relationship between the degree of segregation, or concentration of African Americans in a school, and low academic achievement (Bankston \& Caldas, 1996). The study also examined the effect of the degree of minority concentration and its impact on African American versus White students. Results indicated the percentage of minority concentration had a powerful negative influence on student achievement test performance and this influence is not attributed to socio-economic factors. In addition, both the degree of minority concentration negatively affects whites and African Americans. From this study, we can glean that largely segregated, urban, low-performing schools with a high percentage of African American students has a negative impact on student achievement for both African American and White students.

Academically and behaviorally, students of color are primarily seen as deficits instead of assets in the classroom. According to research, many classroom teachers have a deficit view toward the continent of Africa and it impacts the way African Americans are viewed in the classroom (Loewen, 2008). Thus, African American students are at a disadvantage due to deeply embedded views of White Americans that see them as "them" or "the other" because they are not of European ancestry (Gabbard, 2000, p. 145). These 'other" races are viewed as inferior, deviant and in possession of genetic deficits. Based upon research that shows they are often viewed as a threat, it is difficult to assume that African Americans in schools with a higher percentage of African American students than White are treated equally when it comes to discipline. 


\section{School Climate and Exclusionary Discipline Decisions}

For over a hundred years, researchers have sanctioned the importance of school climate (Perry, 1914). School climate encompasses the quality and character of school life and is based upon people's experiences (Cohen, McCabe, Michelli \& Pickeral, 2009). Further, school climate refers to the norms, values and expectations that create a welcoming environment where students, families and educators work together toward a common school vision. A positive school climate is necessary for students to feel engaged and accepted to perform well academically and obey school rules. Unfortunately, school climate can either be positive or negative and the results have a profound impact on students.

A positive school culture is one that treats all students with respect and teachers and administrators sincerely care about the success of students. Students are very perceptive of teacher apathy toward them and as soon as they know their teachers don't believe the rewards of education - knowledge, skills, admission to college and access to a good paying job- are available for them, students have few reasons to abide by school rules (Noguera, 2003). These students are often labeled as being on a trajectory for ending up in prison.

Numerous students attending high poverty schools in California felt that educators perceived them as 'animals', 'inmates' or 'killers' (Fine, Burns, Payne \& Torre, 2004). Additionally, the same study found that black male and female students are less than half as likely as white students to believe their teachers support them and care about their academic success (Noguera, 20003). Sadly, educators often view students from low socio-economic status, African American students and low performing students, as not 
fitting into the norms of the school and these students are frequently and unfairly targeted for suspension.

Schools norms, educational expectations, school and classroom practices and policies reflect the values of those who create them. Teacher preparation programs are not preparing pre-service teachers with culturally responsive pedagogy and this results in the status quo of the mainstream culture accepted as the norm. Thus, white middle class individuals overwhelmingly make disciplinary decisions tainted with cultural norms that result in higher percentages of disciplinary referrals and exclusionary disciplinary practices toward African American males (Monroe, 2005). This further results in school disciplinary practices that resemble strategies utilized to punish adults in society.

Educators often label students who frequently violate school rules as defiant, maladjusted and difficult to deal with (Brookover \& Erickson, 1969). In addition, these same students are more likely to internalize negative labels placed upon them by the same educators entrusted with creating a school culture that welcomes and celebrates their diversity and act out according to the expectations teachers set for them (Johnson, 1995). An abundance of research has shown that labeling and exclusionary discipline practices can create a self-fulfilling prophecy resulting in a perpetual cycle of antisocial behavior that increasingly becomes difficult to break (Casella, 2001).

As students get older, their cycles of misbehavior are increasingly considered criminal and students find themselves on the school to prison pipeline. More, public schools increasingly operate like prisons rather than schools complete with guards, metal detectors and surveillance cameras to monitor and control students (Noguera, 2003). 
For over forty years, researchers have established that African American males are overrepresented in exclusionary discipline consequences of suspension and expulsion (The Children's Defense Fund, 1975). Suspension and expulsion are the most widely used prescriptions for student discipline issues despite research findings that these policies are not only ineffective, but they exacerbate the discipline problems they seek to reduce (Mayer, 1995; Sugai \& Horner, 2002). In a study that analyzed school discipline data from a large, urban, Midwest school to determine whether African American youth engaged in more severe behaviors thus making severe disciplinary actions justified, researchers found African American youth received disproportionate referrals for subjective and nonviolent offenses, such as excessive noise and disrespect (Skiba, Michael, Nardo \& Peterson, 2000).

Historically, African American males have been on the receiving end of an overabundance of their share of punitive exclusionary consequences including corporal punishment. Ironically, the overrepresentation of punitive consequences was not administered due to engagement in severe behaviors (McFadden, Marsh, Price, \& Hwang, 1992: Shaw \& Braden, 1990). The punishments were simply arbitrary and unjustified by school documented behavior or discipline data. It is this uneven identification of African American students in exclusionary discipline that continues to create opportunities for additional research to determine what discipline strategies work to improve student behavior for all students.

\section{Zero Tolerance Policies}

Despite the widespread use of exclusionary discipline practices, i. e., suspension, student behavior issues continued to increase. In the early 1990s, zero tolerance discipline 
policies grew in popularity in response to a national need for stiffer approaches to school discipline problems. In 1994, the Gun Free Schools Act exacerbated zero tolerance policies because schools were mandated to adopt zero tolerance policies for weapons (Simon, 2007). These same policies resemble adult penalties in the criminal justice system for infractions such as alcohol, tobacco, drugs and violence in schools and left administrators with very little room to apply mitigating circumstances (Schwartz \& Reiser, 2001).

Johns, Boydon and Pittz (2001) reported that 94 percent of public schools in the U.S. has adopted zero tolerance initiatives. Despite the high percentage of implementation by schools, analyses of zero tolerance policies began to raise issues of racial discrepancies in the use of the policy. Skiba and Peterson (2000) found that zero tolerance policies were riddled with ambiguous descriptions of the difference between minor offenses and severe offense. This ambiguity opened the door for critics and those opposing zero tolerance policies to point to the unfair application of the policy toward atrisk students, mostly African American males.

In 1994, the Gun Free Schools Act exacerbated the problem of student exclusionary discipline policies because schools were mandated to adopt zero tolerance policies for weapons (Simon, 2006). An argument could be made that zero tolerance policies ushered in the beginning of the criminalization of schools evidenced by the daily presence of police officers in schools. In 2004, a national teacher survey reported that 67 percent of teachers working in majority African American or Hispanic secondary schools had armed police officers stationed in their schools (Public Agenda Foundation, 2004). 
It did not occur by accident that schools began to function more like prisons. Since 1992, 45 states have enacted laws making it easier to prosecute juveniles as adults, 31 states have implemented harsher sanctions against juveniles for a variety of offenses and 47 decreased confidentiality requirements for juveniles (Osher, Quinn, Poirer, \& Rutherford, 2003). The criminalization of schooling was now the norm in most schools in the United States and with this new trend, schools began to look more like prisons that institutions of learning. In urban, high minority schools, metal detectors, police officers, security officers, gates, walls and barricades became commonplace (DeVoe et al., 2004). In contrast, rural and suburban schools with predominantly white student populations became more criminalized through the implementation of drug sniffing dogs.

Zero tolerance policies ushered in the criminalization of schools turning them into pipelines to prison for a disproportionate number of students of color. These policies existed to exclude low-performing, disruptive students, and overwhelmingly African American male students. The policy succeeded because it strengthened the power of school administrators to utilize school police officers to exclude an unwelcome population from the school and more likely, from standardized test scores (Bowditch, 1993). Over time, the sustained research showing the overrepresentation of African American males and minorities in zero tolerance policy enforcement created a call to action for more research on policies and practices to mitigate the subjective use of zero tolerance policies.

\section{Policies and Practices Seeking to Mitigate Exclusionary Discipline Practices}

In response to data identifying the disproportionate use of exclusionary discipline practices with minority students, schools began to collect information to identify and 
understand the potential underlying causes of the disproportionality. One possible cause of the disproportionality was identified as a cultural gap between teachers and the students' beliefs of appropriate behavior, lack of training in culturally responsive teaching and behavior management strategies and personal biases fueling discriminatory actions (Rafaele Mendez \& Knoff, 2003). School districts began to provide diversity or cultural competence training for teachers to minimize racial bias and/or discriminatory policies and practices unfairly targeting minority students. This training was tantamount to creating inclusive and non-discriminatory environments for students because the majority of educators are European Americans who have not experienced significant interaction with individuals outside of their one racial group (Milner, 2006). Instructional training and techniques included professional development suggesting teachers become aware of their own cultural biases, the cultures of their students, families and the communities they serve (Sugai et al., 2012).

This approach to leveling the playing field for minority overrepresentation in exclusionary discipline and zero tolerance policies garnered national support as school teams began to examine their own learning histories and study the context in which certain behaviors were evident. The end goal became understanding the cultural differences in behavioral expectations at home and school and considering these differences in behavioral definitions and not student ethnicity (Monroe, 2005). In additional to culturally responsive teaching, other strategies such as restorative practices were implemented to minimize the overuse of exclusionary discipline practices with minorities. 


\section{Restorative Justice}

Restorative justice is a framework for juvenile justice reform that seeks to involve offenders, victims and their families, citizens and community groups to effectively respond to juvenile crime. Restorative justice encompasses practices in which approaches to crime focus on repairing the damage done and giving a voice to the victims (Bazemore \& Umbreit, 2001). Historically, juvenile justice systems focused on identifying the culprit, determining what law was broken and how to punish or provide treatment for the offender. According to Zehr (1990), restorative justice focuses on three main questions: (1) What is nature of the harm or crime (2) What can be done to repair the damage resulting from the harm? And (3) who is responsible for making it right?

In education, the term restorative justice is more well-known as restorative practices and, in the school setting, wrong doing or misconduct of students is not viewed as rule breaking but as a violation against people and relationships in the school and the neighboring community (Cameron \& Thorsborne, 2001). Restorative practices in schools have been referred to as not tough enough and students take advantage of educators'

efforts to ensure opportunities to fix problem behavior. Positive Behavior Interventions \& Supports is closely related to restorative practices and is discussed in detail in the next section.

\section{Positive Behavior Interventions \& Supports}

School-wide Positive Behavior Interventions and Supports (SWPBIS) is a systems approach to creating the social culture and behavior supports necessary for all students in a school to achieve both social and academic success. SWPBIS has been shown to decrease disciplinary problems and increase student academic achievement 
across elementary, middle and high schools (Bohanon et al., 2006). The seminal article on Positive Behavioral Interventions and Supports (Sugai et al., 2000) defines PBIS as the application of a behaviorally-based systems approach to increasing the capacity of schools, families, and communities to create effective environments that improve the connection between validated best practices research and the environments where teaching and learning take place.

To address the waxing problem of student misbehavior in schools, the Individuals with Disabilities Education Act (IDEA) of 1997 established the inclusion of positive behavioral interventions and supports into discipline policy, practice and classroom behavior management in all U.S. schools (Sugai \& Horner, 2002). Positive Behavior Interventions and Supports (PBIS) comprises universal school-wide strategies intended to facilitate success for $85 \%$ to $95 \%$ of students and targeted small-group or individual interventions are employed for those $5 \%-15 \%$ of students who do not achieve success through universal strategies (Sugai et al., 2000).

PBIS is a proactive system of school-wide behavior management that focuses on four key areas. Schools must identify clear and measurable outcomes; collect and use data to guide their decision making; implement research-based practices; and invest in systems to ensure those practices are implemented with fidelity and sustained over time (Simonsen, Sugai \& Negron, 2008). Additionally, PBIS emphasizes the utilization of data collection and analysis to anticipate antecedent situations and prevent problem behaviors, increasing student social and communication skills to eliminate triggers and teaching replacement social skills and behaviors resulting in meaningful and sustainable changes in student behavior. Thus, teaching becomes the change tool to successfully implement 
PBIS and the focus shifts to adjusting adult behavior (e. g., responses, routines, instructional expectations) and creating an optimal learning environment where all students are successful (Sugai, et al., 2000).

PBIS utilizes a three-tiered approach to behavior support system designed to address the behavioral needs of all students while providing a focus on those students who need additional interventions and supports (Sugai \& Horner, 2002). Each tier of the PBIS model targets specific groups of children and, while most students fall within the first tier, students who fall within Tier 2 or Tier 3 are afforded various levels of additional support. Tier 1 of PBIS requires the creation of a school-wide or universal behavior management plan to address discipline issues in the school. The foundation of the plan should be a research-based program that promotes positive behavior approaches to discipline and is actively practiced by all administrators, teachers and staff members. This program is intended to address approximately $80 \%$ of behavioral issues in a school, and, if implemented consistently, builds a culture and school climate that sets the foundation for the remaining tiers of PBIS to be implemented successfully (Sugai \& Horner, 2002).

Tier 2 PBIS interventions are employed when a student regularly displays challenging behaviors despite the implementation of school-wide Tier 1 behavior management plans and strategies. Tier 2 interventions encompass approximately $20 \%$ of students requiring more intentional and focused plans for behavior management. Students who need additional supports are often identified through a data system that monitors the number of office discipline referrals a student receives and creates the data necessary to trigger the need for additional behavioral supports (Riffel, 2011). Once identified, the 
teacher and administrators provide more specific positive behavioral techniques to address the struggling student and maintain order in the classroom environment to help the student experience more time in the classroom and overall success in school.

Tier 3 PBIS interventions are specifically reserved for those students who manifest the most severe and resistant behavioral challenges, generally about $5 \%$ of the student population (Riffel, 2011). Interventions at Tier 3 level require a further increase in intentional collection of behavior data over an extended period to make data-based decisions to improve outcomes for the student. Routinely, Tier 3 interventions can be implemented within the regular classroom setting, but, the success rate is conditional based upon how consistently Tier 1 and Tier 2 interventions have been implemented within the classroom and throughout the entire school. The ensuing issue is how to achieve successful school-wide implementation of PBIS with teacher fidelity and buy-in to accomplish the goal of creating effective "host environments" that support the use of proactive discipline policies (Sugai \& Horner, 1994).

\section{Positive Behavior Interventions and Supports Implementation}

There are four key elements of Positive Behavior Interventions and Supports: outcomes, shared fundamental support systems and framework, research-based practices and the implementation of data driven, validated practices to ensure sustainability and generalized effects. (Sugai et al., 2000). The overarching goal is to provide a broad range of systemic and individualized strategies for preventing problem student behaviors.

Successful school-wide implementation of Positive Behavior Interventions and Supports includes six common features: statement of purpose, clearly defined expectations, procedures for teaching expectations, promoting expected behaviors, 
preventing problem behaviors and data collection for decision making (Sugai \& Horner, 2002). A comprehensive model including these six features is necessary to provide a web of supports that "wrap around" children and their families to break cycles of separation from school-wide improvement programs (Sugai et al., 2000).

Following the adoption of the four key elements and the six common features of PBIS, Sugai and Horner (2002) recommend implementing the program in a five-step process. The first step is to establish a leadership team inclusive of teachers who have strong classroom management and behavioral competence, possess leadership qualities, have the respect of their colleagues and represent a multitude of grade levels and departments. This team is responsible for identifying the as-is state of the behavioral needs of the school and reviewing school improvement action plans. Next, if schools intend to establish a school-wide PBIS policy, it is imperative that administrators secure long-term (3-4 years) buy-in from at least $80 \%$ of the teachers and staff. After identifying key players and securing staff buy-in, it is the responsibility of the administrators and the school leadership team to develop an action plan through the utilization of data (attendance, discipline referrals, suspensions and behavior incidences) to determine priority improvement areas.

According to Sugai and Horner (2002), administrators should provide all teachers and staff with professional development and training supports prior to the implementation and ongoing support in PBIS practices and strategies. Step five suggests that administrators and the leadership team analyze data (referrals, suspensions, attendance, etc.) to determine the success of the outcomes of the plan. The leadership team should 
meet at least bi-weekly to analyze data and discuss next steps to maximize the successful implementation of the behavioral intervention program.

If PBIS is in fact an effective program for reducing student discipline issues in low-performing, urban, public schools, why are many of these same schools still struggling to decrease or eliminate student behavior problems? A possible hypothesis is the inadequate implementation of PBIS. In fact, evidence suggests that effective strategies to improve the behavioral climate for students remain significantly underutilized in public schools (Skiba \& Peterson, 2000). Despite evidence promoting effective behavioral strategies, i.e., PBIS, researchers suggest the underutilization is due to resistance from teachers and school personnel (Axelrod, Moyer, \& Berry, 1990). Still, other researchers blame the lack of utilization on poorly implemented behavioral strategies (Skiba \& Peterson, 2000).

The implementation of PBIS is inclusive of a continuum of behavior support practices and systems (Sugai \& Horner, 2009). In essence, teachers should be supported with professional development at every stage along the way during the school-wide implementation process. Research on the PBIS framework places high importance on embedded and continual professional development, including monitoring each phase of implementation (Sugai, Horner, Fixsen \& Blase, 2010). It is essential that teachers receive the tools and training necessary to ensure successful implementation of the PBIS framework, but, unfortunately there are many obstacles to overcome to ensure successful school-wide PBIS implementation. Thus, the issue of the efficiency of implementation continually resurfaces and provokes questions about whether teachers have buy-in with inadequate training or have no buy-in regardless of the adequacy of training received. 
An examination of studies on the implementation of Positive Behavior Interventions and Supports identified several studies, both quantitative and qualitative, seeking to identify positive and negative issues with the implementation of PBIS. One such study surveyed teachers to determine if the implementation of PBIS positively impacted student math and reading scores and lower rates of truancy (Pas \& Bradshaw, 2012). Results showed that school-wide PBIS implementation positively impacted academics and truancy rates, but did not include teacher interviews and perspectives on the specific key elements from which to attribute success. Another qualitative study, researchers interviewed fourteen educational consultants to ascertain barriers that impede school personnel's commitment to the implementation of PBIS and how the leadership team worked together to design and implement interventions (Lohrmann, Forman, Martin \& Palmieri, 2008).

One case study examined the PBIS implementation in the Florida Positive Behavior Support Project which utilized a systematic review process to identify and understand supports and barriers to the successful implementation of school-wide PBIS using measures of the degree of implementation i.e., high or low fidelity and how the project could impact barriers and facilitators. (Kincaid, Childs, Blasé \& Wallace, 2007). Each of the 26 participating school teams completed a Benchmarks of Quality questionnaire to determine if their respective schools were implementing with high or low fidelity. Findings indicated schools implementing PBIS with low degrees of fidelity identified practical, operational types of barriers while schools implementing PBIS with high degrees of fidelity reported struggles with systems issues. Both low-implementing and high-implementing schools perceived that staff buy-in was the area with the greatest 
barrier of positively impacting effective PBIS implementation followed by the use of data, ineffective implementation, lack of reward systems and implementation issues. I commend the researchers for identifying barrier and facilitator themes to highlight patterns of issues working for and against effective PBIS implementation. This case study is similar to the research proposed here, but the voices emanate from groups of teams from each of the participating schools and, once again, not the teachers on the front line.

Further, the Florida Positive Behavior Support Project study sought to determine specific barriers impeding the implementation of school-wide PBIS, but interviews were conducted with educational consultants (self-employed consultants, private for-profit consultants, university faculty, etc.) instead of teachers. While the study yielded valuable information, the educational consultants are not the front-line stakeholders leading the charge to implement PBIS. A gap in the research exists due to the lack of teacher voice in the school-wide PBIS implementation process and the overall effectiveness of PBIS in urban, low-performing schools. The goal of my qualitative research study is to interview teachers to gain and in-depth understanding of their perceptions of obstacles and supports to the successful implementation of PBIS in urban, low-performing schools. In essence, how and why are PBIS research and implementation policies at odds with the actual implementation and practice of PBIS in schools?

\section{Positive Behavior Interventions and Supports Effectiveness}

School-Wide Positive Behavior Support (SWPBS) has become a popular approach to manage student behavior in schools, but some researchers assert several issues must be addressed to determine the effectiveness of this approach (Brandt, Chitiyo $\&$ May, 2014). At issue, is the appropriateness of the different measures used to 
determine the efficacy of SWPBS. Brandt, Chitiyo and May (2014) posit many schools are using more indirect measures (record reviews, rating scales) to determine the effectiveness of SWPBS implementation and there is a need for supplementary research to determine which measures more accurately reflect the efficacy of the approach.

Office discipline referrals (ODRs) are the most common measure used to monitor and analyze problem behavior in SWPBIS (Bohanon et al., 2006). ODRs are forms that document an occurrence of problem behavior by a student and can be used to record behavior data for individual students and to gather comparison data that can be used to compare students within the school or district-wide (Brandt, Chitiyo \& May, 2014). Office discipline referrals are often associated with specific behaviors such as classroom disruption, defiance, fighting, threat/assault, bus problems and destruction of property (Luiselli, Putnam, Handler \& Feinberg, 2005). Data-based computer programs, such as the School-Wide Information System (SWIS), are web-based programs that collect and summarize ODR data for elementary, middle and high school students, and are often used by schools to ease the recording and tracking of student ODRs (Brandt, Chitiyo \& May, 2014).

The use of ODRs as a measure of PBIS effectiveness have both strengths and weaknesses. McCurdy, Mannella and Eldridge (2003) found schools implementing SWPBS components with fidelity are likely to see decreases in ODRs. Additionally, some researchers have found that ODRs were prescriptive of overall school climate and schools with higher numbers of ODRs often had more issues with school climate (Irvin, Tobin, Sprague, Sugai \& Vincent, 2004). Conversely, the literature contends there are 
concerns about the use of ODRs to measure the effectiveness of the school-wide application of PBIS.

First, to determine the effectiveness of SWPBS to improve student behavior, it is necessary that schools collected baseline data prior to SWPBS implementation to compare to any changes that may occur after SWPBS implementation. Unfortunately, many schools are rendered unable to compare ODR data prior to SWPBS implementation due to inconsistent, inaccurate or the absence of baseline data (Luiselli, Putnam, Handler \& Feinberg, 2005). Second, and extremely important to this qualitative study, researchers have found that a reduction in office discipline referrals (ODRs) does not necessarily correlate with an increase in prosocial behavior (Lassen, Steele \& Sailor, 2006; Warren et al., 2006). In addition, the same researchers found that decreases in ODRs could just as easily be attributable to school policies, i.e., administrator instructions, on what constitutes reportable behavioral infractions and/or differing levels of teacher tolerance to less severe student disruptions or problem behaviors.

Obviously, this creates a lack of consistency in the accuracy of using ODRs as a single measure of SWPBIS effectiveness to increase student prosocial behavior. In addition, the use of ODRs to measure problem behaviors may exclude students who internalize problem behaviors as ODRs are typically used to report and measure externalized behaviors. Students who internalize behaviors may not necessarily or repeatedly act out, which may limit teacher reports and limit the effectiveness of ODRs to monitor all student behaviors within a school.

Lastly, ODRs, used as a singular measure of student problem behaviors, may not be sensitive enough to measure functions of behavior for individual students. Brandt, 
Chitiyo and May (2014), posit that combinations of measurement systems are more beneficial than relying on a single measure of problem behaviors. Furthermore, Marchant et al. (2009) determined using a combination of ODRs and behavioral rating scales provides useful data for decision making. Essentially, research overwhelmingly supports the use of multiple data points to determine the effectiveness of PBIS to reduce problem student behaviors. Thus, this qualitative study seeks to fill a gap in the literature calling for the need for more studies on PBIS in urban school environments and utilizes multiple data points including teacher and principal perceptions of the effectiveness of the SWPBIS framework to improve the overall school climate and reduce problem student behaviors.

\section{Teachers' Perceptions}

An in-depth understanding of teachers' perceptions of behavior is essential to meet the challenges of being proactive, identifying problem behaviors, and providing early intervention (Tillery, Varjas, Meyers, \& Collins, 2010). Not surprisingly, teacher concerns and complaints about student discipline have been occurring for nearly a hundred years. In the early 1930s, qualitative researchers reported that new teachers in England complained extensively about issues with student discipline, insufficient materials and equipment, social context of the school location, depressed neighborhoods and improper treatment of teachers from aggressive parents (Phillips, 1932).

\section{Pre-service Teacher Perceptions}

Decades later, a study of female pre-service teachers in the United States utilized three sentence completion stems to ascertain teacher concerns prior to and after student teaching. Findings indicated teachers were most concerned with student discipline and 
being esteemed by their students (Travers, Rabinowitz \& Nemovicher, 1952). Similar studies of elementary and secondary student teachers echoed similar concerns about student discipline and how their students would respond to them (Thompson, 1963). In this study, the sample size consisted of 125 student teachers near the completion of student teaching and Thompson's research instrument was a 35-question checklist.

In addition, a similar study analyzed survey data collected from 133 first year teachers in Indiana and Texas found the most frequently reported concern (36\%) was student discipline (York, 1968). Correspondingly, both pre-service teachers engaged in varying phases of completing their student teaching assignments and first year teachers reported higher incidences of concern related to student discipline. Thus, prior to beginning their teaching career, pre-service teachers expressed anxiety over student discipline and those concerns remained evident during the first year of teaching.

Fuller (1969) conducted a study to examine the concerns of small groups of preservice teachers to determine if their developing concerns changed over time from preservice to experienced teachers and if their concerns could be conceptualized or perceived in purposeful ways. After interviewing teachers prior to student teaching, early in their teaching career and after gaining years of teaching experience. Fuller posited a three-phase developmental conceptualization of teacher concerns and concluded that teacher concerns can change over time and teachers who retain their early concerns regarding student behavior may leave the teaching profession at higher rates that those who do not. Not surprisingly, student behavior concerns ranked at the forefront of teacher concerns before student teaching and early into their teaching career. 


\section{Experienced Teachers}

More experienced teachers were less concerned about student behavior, but experienced teachers continue to acknowledge student discipline as an area of concern. More recently, research indicates lack of teacher support for SWPBIS may contribute to implementation challenges. Specifically, leadership teams and SWPBIS facilitators have identified teacher support for SWPBIS as a significant factor in the implementation process (Kincaid, Childs, Blasé \& Wallace, 2007; Lohrmann, Forman, Martin \& Palmieri, 2008). Teachers are the central stakeholders responsible for implementing SWPBIS with fidelity and, if successful implementation of SWPBIS is largely dependent on data from teachers, it is essential to gain a fundamental understanding of teacher perspectives to prevent or mitigate issues that could thwart the implementation (Feuerborn, Wallace \& Tyre, 2016).

Collier and Henriksen (2012) studied teachers' perceptions of a multiple high-risk behavior prevention program and found that teacher motivation, comfort level, and positive perception regarding the approach of the program were critical toward successful implementation. In addition, results indicated targeting and enhancing teachers' perceptions in five areas would increase the effectiveness of the implementation process. The five areas are (a) the public's perception of the school, (b) the school's positive changes to address high-risk behavior in the students, (c) the school's positive changes to address preventing practices that may increase high-risk behavior in students, (d) the effectiveness and consistency of the current program, and (e) the effectiveness of the program's mental health professional (Collier \& Henriksen, 2012). 
Feuerborn, Wallace and Tyre (2016) conducted a qualitative exploratory study of middle and high school teacher perceptions to gain a better understanding of teacher perceptions and needs across varying levels of SWPBIS implementation. The study took place in western Washington and included 19 public schools (four urban, three, rural and two suburban) across nine school districts in various stages of the SWPBIS implementation process ranging from readiness to full implementation. Study participants included general and special education teachers who work directly with students and researchers. The instrument used to obtain teacher concerns consisted of a qualitative needs assessment method adapted from the concerns-based adoption model (CBAM), which is a stage-based theory of educational change used to understand stakeholders' concerns to enable change agents to intervene and provide the support necessary to address the identified needs (Hall \& Hord, 2011).

Findings revealed the most prevalent teacher concerns were related to staff support and consensus with the most prevalent need identified in the area of collaboration with teacher responses to open-ended interview questions including statements such as “There isn't enough understanding and buy-in to the PBIS program here, and that leads to a lack of consistency that will sink the program" and "unless we are all on board" (Feuerborn, Wallace \& Tyre, 2016, p. 4). Further, a total of nine themes emerged from the study and, in addition to collaboration, included teacher concerns regarding: (a) disciplinary consequences, (b) administrator support, (c) consistency, (d) specific procedures for handling discipline issues related to the school-wide plan, (e) defining and teaching expectations, (f) student-teacher relationships and (g) a system to acknowledge or reward students for meeting behavioral expectations. 
In summary, staff buy-in is an essential component for any intervention geared toward transforming school practices (Handler et al., 2007). The afore-mentioned studies demonstrate the value of understanding teacher concerns regarding SWPBIS and to identify factors that facilitate successful implementation and factors that create barriers to successful SWPBIS. In the field of education, it is extremely important to implement research-based practices, such as in the case of SWPBIS, to create positive change. Unfortunately, utilizing a research-based practice constitutes only half the battle. The remaining challenge lies in understanding the insights, concerns and needs of the teachers and staff members being asked to change. Teacher concerns regarding student discipline have remained prevalent for decades, continue to persist and have recently been exacerbated by national, state, district and school-wide pushes to implement Positive Behavioral Interventions \& Supports (PBIS) in public schools to replace the punishmentbased zero tolerance policies that have dominated discipline policies for the past few decades.

\section{Principals' Perceptions}

In today's educational system, public school principals are ultimately held responsible for the academic success or failure of their students and for maintaining safe school environments (Richter, Lewis \& Hagar, 2012). In addition, principals play a significant role in helping to influence teacher outcomes, such as job satisfaction, attitudes, collegiality and commitment to the school (Markow \& Cooper, 2008; Price, 2011). Furthermore, research has shown strong principal leadership and support are directly related to the degree to which a practice is implemented as intended, or the implementation fidelity, of a practice (Payne, Gottfredson \& Gottfredson, 2006). 
A study by Kam, Greenberg and Walls (2003) explored the implementation and outcomes of a social-emotional learning curriculum and they found improved student outcomes were only apparent in schools in which both principal support for the curriculum and fidelity of implementation were high. Thus, even with a high fidelity of implementation, student outcomes did not improve when principal support was low.

Principal support has also been shown to be a critical component for practice sustainability (Andreou, McIntosh, Ross \& Kahn, 2015). A study conducted by McIntosh et al. (2014) found school personnel rated strong administrator support as the most important factor influencing sustainability of a school-wide approach to behavior. In contrast, the lack of principal support has been shown to be a barrier to practice sustainability. In a 2008 study by Lohrmann, Forman, Martin and Palmieri, researchers analyzed survey responses from a large, national sample of 257 school team members who completed the School-Wide Universal Behavior Sustainability Index.

Findings indicated that without administrator support, practices would likely fail to achieve full implementation and, consequently, fail to achieve sustainability. Principal support has been shown to be important in school-wide PBIS and PBIS training emphasizes the importance of the inclusion of administrators in the process of planning and implementation (Sugai et al., 2010).

Specifically, Sugai et al. (2010) recommend the principal be an active member of the PBIS leadership team to promote successful school-wide PBIS implementation because their unique role in the school allows them to allocate resources such as teacher training, time and funding to ensure success. The active support of principals for PBIS has been shown to either help or hinder the success of successful school-wide 
implementation and sustainability and the absence of principal support may be related to other barriers such as (a) low conceptual understanding of PBIS among staff, (b) difficulty balancing competing initiatives, (c) shortage of planning time, (d) and greater dependence on technical assistance (Debnam, Pas \& Bradshaw, 2013).

A study by McIntosh, Kelm \& Delabra (2016) utilized in-depth interviews of ten school administrators to explore factors that influenced principal support of school-wide PBIS. Qualitative data analysis using the Enhanced Critical Incident Technique revealed eight helping and three hindering categories of experiences that influenced principals' change in support for school-wide PBIS. Helping categories generated from the interviews include (a) learning from others, (b) learning how PBIS aligns with personal values, (c) experiencing its effectiveness firsthand, (d) observing a need for PBIS, (e) attending informative PBIS trainings, (f) staff support, (g) connection to coach, and (h) attending PBIS team meetings

Hindering factors include (a) disagreement with philosophy or PBIS, (b) witnessing unsupportive staff and (c) negative reaction to time commitment. The participant responses also generated a Wish list, which included a desire to learn more about PBIS prior to implementation and planning for implementation.

School principals play a pivotal role in adopting, implementing and sustaining PBIS practices in schools. Their support, or lack of support, can make the difference between successful implementation of SWPBIS and outright abandonment of the framework entirely. Because research shows principal support for the implementation and sustainability of school-wide practices plays an important role in whether SWPBIS succeeds or fails, it is extremely important to include principal perceptions when 
exploring teacher perceptions of the SWPBIS framework to gain full insight regarding issues of collaboration, communication, effectiveness, implementation and sustainability.

\section{Summary}

Research shows low-performing schools share certain commonalities such as community poverty, low expectations for student achievement, high teacher absenteeism and high rates of teacher turnover (Corallo \& McDonald, 2001). Recall that urban, lowperforming schools suffer from high rates of disciplinary problems, violent behaviors and suspensions, in addition to the challenges of poverty (Handler et al., 2007).

Sugai and Horner (2008) posit that school-wide positive behavior interventions and supports (SWPBS), which includes evidence-based interventions, practices and processes, provide structure and organization for schools to achieve effective, efficient and relevant approaches to preventing chronic problem behavior in schools. One caveat to this assertion is the call for more research on the effectiveness of PBIS in urban school settings by the authors of the seminal article in PBIS research, Sugai et al (2000).

Further, Sugai and Horner (2008) suggest we still need to learn about what works and does not work with SWPBS; specifically, additional questions surrounding improved implementation effectiveness, sustained fidelity of implementation of effective practices and expanded use across more and different schools, districts and states. Urban public schools are different schools because they deal with challenges that are especially daunting for disadvantaged communities and schools must meet every need of their students, where the need directly impacts student learning (Warren et al, 2003). Even

more directly, Sugai and Horner (2008) ask how can SWPBS be extended and integrated within high schools, complex urban school settings, preschool and early childhood 
settings, structured classroom contexts, alternative problems and early interventions programs.

In addition, (McCurdy, Mannella \& Eldridge, 2003) cite the need for more research on PBIS in urban, high poverty environments. Tillery, Varjas, Meyers \& Smith Collins, (2010) state there is a need for in-depth data regarding teachers' perceptions and approaches to behavior management to improve training practices for effective implementation of school wide PBIS, management and intervention. To address the aforementioned gaps, this research study seeks to extend what is already known in the research literature about the effectiveness of PBIS and provide teacher and principal voice on SWPBIS effectiveness in the context of complex, urban, low-performing schools. 


\section{CHAPTER III METHODOLOGY}

The methodology of the present study is outlined in this chapter. The purpose of this study was to employ qualitative research methods to examine teachers' and principals' perceptions of the effectiveness of Positive Behavior Interventions and Supports (PBIS) in low performing schools in a large, urban school district in the Southeast Region of the United States. This chapter provides a research-based rationale for electing to conduct a qualitative research study to answer my research questions. In addition, this chapter entails an overview of the case study method selected for this qualitative research study, data collection and data analysis procedures, foreshadowing of how the findings will be presented/discussed.

\section{Research Questions}

The specific research questions addressed in this study are:

- How do teacher and principal perceptions of School-Wide Positive Behavior Interventions and Supports impact its effectiveness?

- What do teachers and principals in urban, low-performing schools perceive as obstacles and supports to SWPBIS implementation, sustainability and effectiveness? 


\section{Research Design}

Qualitative research methods are appropriate when exploring a problem or an issue, to identify variables not easily determined or to hear silenced voices (Creswell, 2013, p.47-48). Miles, Huberman \& Saldana describe the strengths of qualitative data as having a focus on "naturally occurring, ordinary events in natural settings" to get a clear sense of what "real life" is like (2014, p. 12). To examine and answer my research questions, I employed the use of a collective case study at an urban, low-performing elementary school. By interviewing several different participants at their work sites, i.e., school location, the information received provides valuable insight into the issue of PBIS effectiveness, implementation and sustainability. Additionally, the study of several different cases qualifies this as a multiple instrumental case study.

Even though case study does not have well-defined and well-structured protocols, case study is one of the most frequently used qualitative research strategies (Yin, 2002). A case study is defined as an "empirical inquiry that investigates a contemporary phenomenon in depth and within its real-life context, especially when the boundaries between phenomenon and context are not clearly evident" (Yin, 2009, p.18). The case study approach is also useful when "you want to cover contextual conditions because you believe they are relevant" and when the researcher does not want to manipulate the behavior of those involved in the study" (Baxter \& Jack, 2008, p. 450.).

Furthermore, the case study approach to qualitative inquiry allows the researcher to explore simple through complex interventions, relationships, communities, or programs and is beneficial for the deconstruction and subsequent reconstruction of various phenomena (Yin, 2003). Yazan (2015) states the Yinian view of case study is an 
empirical inquiry that investigates the cases or cases to determine the "how" or "why" questions related to the phenomenon of interest. The present case study seeks to determine how teachers perceive the effectiveness of PBIS in urban, low-performing schools and, therefore, lends itself to the Yinian view.

Robert Yin, Sharan Merriam and Robert Stake are three seminal authors who provide researchers with a road map of procedures to follow when conducting case study research (Creswell, Hanson, Plano \& Morales, 2007). This research would be remiss without the examination of the two other foundational methodologists in the field of case study research, Stake and Merriam. Stake (1995) posits four distinguishing characteristics of qualitative case studies emphasizing that researchers should conduct research that is: holistic (consider interrelationships), empirical (based upon field observations), interpretive (intuition based) and emphatic (reflects vicarious experiences of the researcher). Lastly, Stake defines a case as a "specific, a complex, functioning thing" in an integrated system which "has a boundary and working parts" (Stake, 1995, p.2).

More specifically, Stake defines a qualitative case study as the study of the particularity and complexity of a single case to understand its action within relevant situations. Merriam (1998) characterizes a case as a "thing, a single entity, a unit around which there are boundaries" (p. 27). She states that a case can be a person, a group, a program, or specific policy and includes a much more comprehensive checklist than Yin and Stake. Merriam advocates for case study to be viewed as a legitimate research strategy and further defines it as a particularistic, descriptive (rich, thick description) and heuristic (illuminating) examination of the "intensive holistic description and analysis of a bounded phenomenon" (Merriam, 1998, p. 27). 
Merriam explains the foundational tenets of qualitative research and seeks to eliminate confusion about the indistinct areas in case study research by specifying what constitutes a case study, when to use it and how it differs from other methods of qualitative research (Yazan, 2015).

In the present study, my goal was to examine urban school teachers and principals as they implement Positive Behavior Interventions and Supports (PBIS) in their classrooms and school. In an effort to identify common patterns and themes among different participants, a case study of urban school teachers and a principal working in a low-performing school provides an ideal fit for this research study. The study of multiple teachers and the principal at an elementary school in an urban school district, each defined as a case, makes this study a collective case study and is an acceptable method for case study research (Creswell, 2013, p.150).

According to Yin (2009), the multiple case study design should use the logic of replication, whereby the researcher replicates the procedures for each case. In short, I employed the same procedures for each case, and more specifically, the interview questions and probes were identical for each participant.

\section{Research Context}

Data for this study was collected from an elementary school in a large, urban, public school district in the Southeast Region of the United States and focused on the experiences of teachers and principals working in the challenging environment of urban, low-performing schools. The school district was selected because it is the largest school district in its state and ranks in the top thirty largest school districts in the United States. The selected school district, Marlowe City School District (pseudonym), MCSD, failed 
to make adequate progress on the most recent state assessment and is now considered a school district that "Needs Improvement".

Despite this rating of needs improvement, district spending per student is $\$ 12$, 257 , which is greater than the state average of $\$ 10,192$. Thus, MCSD spends more money per student than the state, but is failing to meet the adequate yearly progress required by state and federal guidelines. MCSD enrolls approximately eighty percent of the students living within the boundaries of the school district to attend one of the district's more than 170 schools. This district employs more than 6,000 teachers to serve the student population of over 100,000 students from pre-kindergarten to $12^{\text {th }}$ grades. The districts' students speak more than 120 different languages and over $60 \%$ of students are eligible for free or reduced-price meals. Close to 47\% of MCSD students are White, 36\% are Black.

Nearly $10 \%$ are Hispanic, almost $4 \%$ are Asian and approximately $4 \%$ identify as Two or More Races. Over $65 \%$ of the district's students qualify for free $(60.9 \%)$ and reduced (4.9\%) lunch. Eighty-four percent of the over 6,000 teachers in the MCSD hold a master's degree or higher and they have on average more than ten years of experience.

MCSD began implementing PBIS in pilot schools in 2013-2014 and expected designated schools to have full implementation of PBIS in the 2017-2018 school year. Thus, teachers and principals selected and interviewed from this district will have at least two years' experience working at a priority school and have received at least two years of training in the implementation of the PBIS framework in their classrooms and in the school-wide behavior plan. 


\section{Sampling Strategy}

Creswell (2007) emphasizes the importance of selecting participants using a sampling strategy such as criterion based, critical case or a variety of other strategies to obtain participants that are willing to provide open and honest information about the research topic. Teachers and principals employed for at least two years at the selected low-performing elementary school in the large, urban, school district where this study takes place qualified as candidates for participation in this study. This study focused on teachers i.e., study participants who share the common dilemma of struggling to implement PBIS in the challenging environment of urban, low-performing schools. Outside of the minimum two years' experience teaching at a PBIS school, no efforts existed to study teachers and/or principals of a specific gender, race or level of education.

Essentially, teacher and principal voice is paramount in this study to help determine if PBIS works to decrease student behaviors that impede classroom instruction and contribute to lower student achievement. Participants' responses provide insight to help the researcher answer this essential research question: How can teacher and principal voice during and after the implementation of PBIS positively impact the future implementation and effectiveness of PBIS to decrease problem student behaviors and discipline issues in urban, low-performing schools?

Interview questions (see Appendix A and Appendix B) focused on initiatives currently in place to respond to student misbehaviors, implementation of the initiatives or practices currently in place, potential facilitators or barriers impacting implementation and teacher and principal perceptions regarding SWPBIS effectiveness to support positive student behaviors and improve the overall school climate. An elementary school 
was selected for this study because most of the research available on teachers' perceptions of the PBIS framework is either focused on middle and high schools (Feuerborn, Wallace \& Tyre, 2016) or focused on elementary teachers' perceptions, but are not situated in an urban context (Tillery, Varjas, Meyers \& Collins, 2010).

The elementary school selected for this study serves students in grades kindergarten through fifth and is located in an urban section of the Marlowe City School District (MCSD). This specific elementary school was designated as a persistently lowachieving school by the state department of education in 2016 for failure to meet state accountability benchmarks for three years in a row. Dorothy Height Elementary School (DHES) has significantly high numbers of office discipline referrals and student suspensions and was selected by the MCSD as a SWPBIS pilot school in the 2014-2015 school year. The selected school is described in detail below:

\section{Dorothy Height Elementary School}

Dorothy Height Elementary School (pseudonym) has a relatively small student population consisting of approximately 270 students in grades kindergarten through fifth grade. The school is located in the downtown area of the city comprised of single-family homes, rental properties, federal housing projects and is plagued by generational poverty, drugs and violence. Dorothy Height Elementary School (DHES) offers a technology magnet program open to students from across the school district, but most of the school's students reside in neighborhoods surrounding the school. Black students $(79.7 \%)$ make up the largest racial group at Height Elementary and White students (11.8\%) constitute the second largest racial group. The remaining population consists of students identified 
as Two or More Races (5.1\%), Hispanic (2.8\%) and Asian and American Indian or Alaskan Native both makeup less than $1 \%$ of the student population.

Every student (100\%) at Height Elementary School receives free lunch, which is well above the district average (60.9\%) and state average of $55.4 \%$. About $11 \%$ of Height's students receive special education services, nearly $13 \%$ of students are homeless, 5\% are English language learners and only 3\% identify as gifted or talented. DHES has one principal, one assistant principal, one school counselor and twenty-six total teachers, inclusive of classroom, special education, interventionists and coaches.

In 2016, the state department of education classified Dorothy Height Elementary School as a school that "Needs Improvement". Due to failing to meet annual measurable objectives (AMO) on state testing requirements for NCLB for three consecutive years, the school received designation as a Priority school by the state. State accountability test scores in reading achievement for the 2015-2016 school year illustrate $62.6 \%$ of students scored novice, $21.3 \%$ scored apprentice, and only $16.1 \%$ of students scored proficient or distinguished. For math achievement in the same school year, 56.1\% of students scored novice, $29.7 \%$ scored apprentice and only $14.2 \%$ of students scored proficient or distinguished.

Non-academic behavior data suggests a sharp increase in suspension data from 21 student suspensions in the 2012-2013 school year to 132 student suspensions in the 20152016 school year. As a result of low academic achievement scores, the principal and staff of Dorothy Height Elementary School currently receive assistance from state turnaround personnel to assist in improving student academic achievement and overall school 
behavioral climate to move the school out of persistently low-achieving status, as measured by state accountability assessments.

For the purposes of this study, the researcher utilized purposeful sampling strategy because it lends itself to the selection of individuals and research sites conducive to providing insight and understanding of the research question(s) driving the study (Creswell, 2013, p. 156). The researcher focused her research on teachers and principals with at least two years' training in the use of PBIS and two years' experience working in the low performing school included in this case study. In addition, this researcher purposefully sought out qualified candidates willing to provide credible information and agree to open and honestly share "their story" (Creswell, 2007, p.133). The sample size goal was approximately 8-10 total teachers and the principal of Dorothy Height Elementary School and this researcher successfully interviewed 10 teachers and the principal. The researcher sent emails and letters to all prospective teachers and the principal of DHES in hopes of identifying subjects interested in participating in this study.

Prospective participants responded via email expressing their interest in participating in the study and, this researcher scheduled one-on-one meetings with each participant before and after school. At the meeting, this researcher present them with the Informed Consent form, which includes information about the study (purpose, methods, duration, risks and benefits) and, following receipt of signed consent forms, the researcher interviewed all 11 participants over the course of two months concerning their perceptions of the SWPBIS framework currently in place at their school. 
Nearly all of the data for this study was collected using teacher and principal interviews to ascertain an understanding of their perceptions and concerns about the SWPBIS framework in their school. Semi-structured interviews lasted approximately thirty minutes and this researcher asked each teacher participant the same 20 open-ended questions prepared in advance with possible probing questions to ensure a focus on answering the central research questions. The principal responded to the same 20 questions, but the focus was primarily on the school SWPBIS framework and not on perceptions of classroom behaviors. Open-ended questions and prompts used in this study were adapted from the Concerns-Based Adoption Model (CBAM), which is a framework for understanding the evolution of educators' questions and concerns during the implementation of research-based practices (Fuller, 1969; Hall \& Hord, 2011; Roach et al., 2009).

\section{Participants}

For the purposes of this collective case study, each participant met the established criteria of at least two years current employment as a teacher or administrator at the selected urban elementary school site. Additional study design criteria required that each teacher or principal participant have at least two years' training in the implementation of the School-Wide Positive Behavior and Interventions (SWPBIS) framework. All 11 study participants successfully met this criteria. Each participant interview followed the appropriate interview protocol based upon the participants' position: Teacher (Appendix A) and principal (Appendix B). A description of each participant in the semi-structured, face-to-face interviews appears in a brief narrative below: 


\section{Wilma}

Wilma is a Caucasian female with 14 years of teaching experience at the elementary school level. She received several years of PBIS training at the school where she formerly taught and two years of PBIS training at DHES. Wilma is finishing her second year as the Science, Technology, Engineering, Art and Math (STEAM) teacher at Height Elementary School (HES). She taught previously at an elementary school in the Midwestern part of the United States, but relocated to the Marlowe City School District (MCSD) a few years ago. Her highest level of education is a doctoral degree in the field of education.

\section{Ellen}

Ellen is a Caucasian female in her second year working as the math interventionist at DHES. She possesses 22 years of teaching experience at the elementary school level in schools located in the Southeastern part of the United States. Ellen has worked in rural school districts for the majority of her years of teaching experience and commutes approximately one hour each way to Dorothy Height from her home in a rural county outside of MCSD. Ellen's highest level of education is master's degree.

\section{Patricia}

Patricia is the magnet coordinator at DHES and is currently in her $27^{\text {th }}$ year of teaching in the Southeastern state where this study takes place. She is a Caucasian female in her third year at DHES and has taught all grades (K-5) at elementary schools located in both rural and urban school districts. The vast majority of her teaching experience, approximately 24 years, took place in a rural setting. In addition to her work with the student technology program, Patricia coaches new and experienced teachers in the areas 
of lesson planning and classroom management. Her terminal degree is specialist in education.

\section{Gayle}

Gayle, a Caucasian female, is a primary (K-2) special education teacher at Dorothy Height Elementary School. She has taught for a total of 12 years in both urban and rural school settings and has been teaching at DHES for approximately five years. Gayle is a member of the Instructional Leadership Team at Height and actively volunteers to help with school functions that increase student and family engagement. She has received PBIS training at Height since the 2013-2014 school year and her highest level of education is master's degree.

\section{Daniel}

Daniel is an African-American, male teacher with 20 years of experience working in elementary and middle schools in multiple states. He has taught for fifth grade for two years at Height Elementary School and has approximately fifteen years experience working as an administrator at charter schools located in the western part of the United States. The students at the charter schools were African-American male students in grades six through eight and the school curriculum focused on economic empowerment. Daniel has received extensive training in PBIS both as a teacher and charter school administrator and is the head coach of the DHES boys basketball team. His highest level of education is master's degree in educational administration with administrator certification. 


\section{Marcy}

Marcy is a second grade teacher finishing her second year of teaching at DHES. She is Caucasian and has fifteen years teaching experience, with 12 of the 15 years consisting of teaching positions in rural counties outside of MCSD. Marcy received training in PBIS as a classroom teacher in the rural district where she taught previously and has received two years of PBIS training at Height. She currently lives in a predominantly Caucasian, rural county outside of MCSD and commutes approximately 40 minutes each way to DHES daily. Marcy's highest level of education is master's degree.

\section{Carla}

Carla is the librarian at Dorothy Height Elementary School. She is a Caucasian, female with nine years of teaching experience, mostly as a fifth grade teacher in a highperforming, mostly white, rural county approximately 20 minutes outside MCSD. Carla was trained in PBIS in her former school states PBIS was a priority district initiative and, thus, professional development in SWPBIS was on-going and intense. Carla's terminal degree is master's in library/media science.
Alice
Alice is a second grade teacher at DHES with two years of total teaching experience, all of which occurred in the urban setting at ubman. Alice is a Caucasian, female who received $100 \%$ of her PBIS training at DHES and states the extent of her PBIS training was delivered via Professional Learning Communities (PLCs) and school- based professional development trainings in the summer and at faculty meetings. Alice's highest level of education attained is master of arts in teaching, which is a master's 
degree program for individuals with bachelor's degrees in fields other than education who wish to obtain teacher certification. In this particular case, Alice's bachelor's degree is in art.

\section{Candace}

Candace is the Goal Clarity Coach, or instructional resource teacher, at Dorothy Height Elementary School. She is an experienced, national board certified teacher and instructional coach with 20 years of teaching experience gained in two states. Candace is a Caucasian, female who has spent most of her teaching career working in urban, lowperforming elementary schools settings teaching students in the primary grades. Her PBIS training includes years of training from her previous school and district and schoolbased trainings for the past two and half years at DHES. Candace's highest level of educational attainment is master's degree in administration with administrator certification.

\section{Arnell}

Arnell is an African American, female Reading Interventionist with nine years of teaching experience all in low-performing, urban elementary schools. Prior to her position as an interventionist, she taught first grade students and currently teaches master's level classes in literacy to teachers at DHES through a partnership with a nearby private university. Arnell received the majority of her PBIS training at another elementary school, but acknowledges she has received PBIS training at Dorothy Height delivered through summer professional development, Professional Learning Communities (PLCs) and faculty meetings. Arnell also acknowledges receiving some 
additional PBIS training from Marlowe City School District trainers and behavior coaches. Her highest level of educational attainment is master's degree.

\section{Norma}

Norma is the principal at Dorothy Height Elementary School. She is an African American female with 19 years of experience in the field of education. Norma began her career teaching fifth grade students at another persistently low-achieving elementary school in MCSD consisting of high need students. However, after a few years, Norma accepted a position as an assistant principal at a high-performing elementary school located in a rural school district approximately one hour away from MCSD. Norma returned to MCSD after a few years and is currently in her fourth year as principal of DHES.

According to Norma, the extent of her PBIS training included training at another elementary school where she worked as an instructional coach and one year of district provided training her first year as principal of Height due to DHES' designation as a PBIS pilot school the prior school year. Norma states, the Marlowe City School District ceased to provide "formal" PBIS training after her first year as principal and she has not received any "formal" district training since mid-way through her second year as principal. Her highest level of educational attainment is master's degree in administration with administrative certification.

As previously mentioned, Fuller (1969) utilized the CBAM model to ascertain the concerns of small groups of pre-service-service teachers to determine if their developing concerns changed over time from pre-service to experienced teachers and if their concerns could be conceptualized or perceived in purposeful ways. Fuller posited a three- 
phase developmental conceptualization of teacher concerns for describing and evaluating teachers' engagement with and implementation of a proposed research-based innovation: (a) Stages of Concern, (b) Levels of Use, and (c) Innovation Configurations (Roach, Kratochwill \& Frank, 2009). In this study, the researcher utilized open-ended interview questions adapted from the CBAM model to ascertain teacher and principal perceptions and concerns regarding the use of the school-wide PBIS framework at a low-performing, urban elementary school.

Qualitative interview strategies can be extremely structured or more spontaneous in nature. For example, the informal conversational interview relies completely on the spontaneous creation of questions during a natural conversation between the researcher and the interviewer (Gall, Gall \& Borg, 2003). This type of interview requires the researcher to construct questions on the fly and while some view this as beneficial because of the lack of structure, others find it unstable due to the lack of consistency in interview questions and the difficulty to code data (Creswell, 2007).

The general interview guide approach allows the researcher some flexibility as they can change the ways the questions are worded and posed to the participant (Gall, Gall \& Borg, 2003). Lack of consistency is an obvious issue with this method and, for this reason, the researcher opted not to use this interview method.

Finally, standardized open-ended interviews are extremely structured regarding the wording of the questions as participants are asked identical open-ended questions that allow participants to provide extremely detailed information and the research to ask probing questions to get more rich data during follow-up questions (Creswell, 2007). Some negatives associated with open-ended interviews include the cumbersome process 
to code the data as the researcher navigates through the narrative responses to identify themes and codes. Positives include the acquisition of rich, thick data and according to Gall, Gall and Borg (2003), open-ended interviews reduce the possibility of researcher bias when the interviewing process involves many participants. For this reason, this researcher utilized semi-structured open-ended interview questions in this study and all interviews took place before or after school to ensure no loss of instructional time for students.

All interviews were face-to face and lasted approximately 30 minutes each. During the interviews, in addition to using a digital recording device, the researcher took notes on a pre-created form 4-5 pages in length to allow enough space to record responses to the interviewee's comments (Creswell, 2013, p. 168). To construct an indepth picture of the cases, this researcher utilized multiple forms of data collection procedures including; interviews, documents review and archival records (Yin, 2009). Interviews were recorded using a recording app on my cell phone and were professionally transcribed verbatim for data analysis purposes. The researcher opted to follow guidelines from McNamara (2009) who suggests the interviewer ask one question at a time, remain as neutral as possible, use occasional head nods and "uh huh's to encourage responses, take notes calmly, use transitions to move between topics and keep the participant focused on the topic without straying to other topics.

Additionally, this researcher was prepared to do some interviews via phone or email if some participants were unavailable for face-to-face interviews, but fortunately all participants made advance appointments to meet for interviews. One limitation of phone interviews is the lack of opportunity to see facial expressions and body language, but, if it 
would have been necessary, the researcher would have employed this strategy. Following the completion of the interviews, member-checking practices took take place by providing study participants a transcript of their interview to read and ensure a true and accurate account of their responses. Participants were instructed to contact me with any questions or corrections, but, if not errors were found, no action was needed on the part of the participant.

\section{Interview Protocol}

Interview questions one through seven provided baseline assumption data of teacher and principal perceptions of what causes students to exhibit positive and negative behaviors. Questions eight through thirteen and seventeen focus on teacher and principal perceptions of issues with SWPBIS implementation, sustainability and effectiveness.

Finally, questions fourteen through twenty focus on teacher and principal perceptions of the overall effectiveness of SWPBIS on decreasing student misbehavior and improving overall school climate.

Upon completion of data collection, pseudonyms were assigned and each participant interview was transcribed in vivo using the online service, Rev.com. Within 24 hours, this researcher received word documents and pdf transcripts of each teacher and principal interview. In first cycle coding, this researcher analyzed participant responses for each interview question to identify codes and patterns in the interview data. After initial coding was complete, this researcher analyzed the data a second time to identify patterns and organized the recurring themes into categories based upon each of the 20 interview questions. 
Through a third round of review, the initial list of twenty categories was combined and narrowed to identify major recurring themes found within each case and compared across all cases within the data. Appendix $\mathrm{C}$ contains a chart that denotes how each code was classified into the themes to ultimately determine findings for each research question.

\section{Additional Sources of Data}

Good researchers are obligated to triangulate, or use multiple methods, data sources, to enhance the validity of research findings (Mathison, 1988). To ensure validity, this researcher intentionally enlisted various methods of data collection including semi-structured interviews, the review of documents such as office discipline referrals (ODRs) and suspension data and TELL Survey (Teaching, Empowering, Leading and Learning) data to reinforce the validity of research data obtained in the study. Suspension data is available publicly on the website of the school district used in this study.

The TELL Survey is a biennial survey that offers every certified educator in the state where this study takes place the opportunity to provide input on teaching conditions that can be used to inform school, district and state improvements. The TELL survey results are available from the state website by school, district and state and assisted the researcher in the analysis of teacher perceptions of student behavior in the school and overall school climate.

Generalizability, also referred to as transferability, refers to the ability to generalize study findings to other settings, populations and contexts (Creswell, 2007). In addition, Creswell states that findings of qualitative research must provide sufficient 
detail from the perspective of the participant to ensure descriptions do justice to their experiences and/or phenomena and transferability with other cases can be assessed. If generalizability is non-existent, the lack thereof is often viewed as a weakness of qualitative methods (Creswell, 2007).

\section{Preparation and Initial Data Analysis}

The final step in the research process is to interpret the data collected during the interview process. Data analysis consists of examining data collected to decipher themes and consists of examining, categorizing, tabulating, testing or otherwise recombining evidence to produce empirical findings" (Yin, 2014, p. 132). In short, the researcher must make sense of the data and organize it into categories or groups of information known as themes or codes (Creswell, 2007). In qualitative research, data analysis entails using a systematic process and specific procedures to achieve accurate and reliable data.

Typical steps include preparing and organizing data for analysis, employing coding strategies to condense the data into themes and representing the data in some type of figure, table or discussion (Creswell, 2013, p. 180). In this study, the researcher utilized this general process to analyze interview and observation data and enlist the use of a conceptual framework to display the central ideas uncovered in the study and how they interact and influence each other (Miles, Huberman \& Saldana, 2013, p.24).

After interviewing the study participants, the researcher enlisted a professional transcription service, Rev.com, to transcribe the interviews In Vivo or word for word using the digital recordings taken during the interviews. Next, the researcheranalyzed or coded the data in what is called first cycle coding. In Vivo coding utilizes words or phrases directly from the participants' own verbiage and identifies them as codes (Miles, 
Huberman \& Saldana, 2013). Charmaz (2001) states that coding is the "critical link" between data collection and the explanation of the meaning of each individual piece of data. Saldaña (2015) posits a code, in qualitative research, is usually a word or short phrase that assigns or captures an evocative meaning for a portion of language-based or visual data. As the researcher begins the coding process, the initial goal is to reduce the amount of data without losing the meaning of the data and ensuring the significant ideas remain.

The second step in the coding process, second cycle coding, focuses on the researcher gaining a more in-depth understanding of the phenomenon and then attempting to develop patterns, categories and themes that emerge from the data. (Miles, Huberman \& Saldana, 2013). Computer software provides assistance in identifying themes and patterns in participant responses to identify barriers and supports to the implementation of PBIS in urban, low-performing schools. The researcher utilized NVivo computer software to assist with first cycle data coding and analysis to uncover common themes and patterns in participant responses. Coding consists of matching key words or tags to segments of text to allow for future retrieval (Miles, Huberman \& Saldana, 2013).

The third step is to identify themes within each case (within-case analysis), and perform a thematic analysis of all the cases, or cross-case analysis, to make assertions and/or interpretations of the meaning of the case. Miles, Huberman and Saldana (2013) identify advantages of researching cross-case or multiple cases is to increase generalizability and provide evidence that the outcomes are evident across many cases. 
In the data analysis of this research study, this researcher performed a thematic analysis of all the cases in an effort to develop assertions applicable to all cases. In the past few decades, many qualitative researchers have opted toward multiple complex cases studies such as those involving teachers and other groups. The researchers' goal has been to identify processes and outcomes across many cases, understand how they are qualified by local conditions and develop more sophisticated descriptions and powerful explanations of the phenomena (Miles, Huberman \& Saldana, 2013; Creswell, 2009; Creswell \& Plano-Clark, 2011). In this study, this is the goal of this researcher.

After transcribing and coding the data, sorting the data into categories, and identifying patterns and themes in the data, the researcher condensed the data and designed a display to help readers draw conclusions from the data (Miles, Huberman \& Saldana, 2013). To display the findings from this study, this researcher utilized a matrix data display to compare data from all interview participants (cases) and compare similarities and differences apparent in the research data. A matrix is composed of rows and columns that show the intersection of two lists and consists of a tabular format that arranges and collects data in one place for easy viewing, detailed analysis and cross-case analysis with other similar cases or sites. Additionally, the matrix display is a visual that requires the researcher to think deeply about the research questions and what parts of the data are required to answer them.

Next, this researcher engaged in deep data analysis, ensuring all relevant information was analyzed thoroughly and organized in a logical manner (Miles, Huberman \& Saldana, 2013). Upon completion of the matrix display model, this scholar employed the prior knowledge gained from literature about the topic, synthesized the data collected in the 
interviews and document analysis, reported the lessons learned from the case and possible implications for future study.

\section{Institutional Review Board}

In this study, the researcher gained access to the study participants by making a request for permission to the Institutional Review Board (IRB), which governs university research studies for human subjects and monitors them to prevent harm or risk to study participants. Next, the researcher filed a research request with the central office gatekeepers of the school district depicted in this study and received permission to conduct the study in HES and conduct interviews with its employees.

Lastly, prior to participant interviews, the researcher provided each study participant with a human subject's consent-to-participate form outlining the purpose of the study and the methods of data collection utilized in the study. The researcher informed participants of their right not to participate, right to confidentiality and the opportunity to review their interview transcripts for authenticity.

All participants signed the informed consent agreement with the researcher and received information regarding the purpose of the study, any risks associated with participation in the study, procedures, duration and benefits to the researcher. During data collection, notes and electronic data remained secured on a password protected device and, after audio transcription was completed, identifying information about work sites and individuals was password protected on a computer kept in a secured area with access by the researcher only. 


\section{Credibility}

In qualitative studies, researchers must establish the study's findings are credible, dependable, confirmable and reliable. Credibility is how confident the qualitative researcher is in the accuracy or truth of the research study's findings. To ensure the trustworthiness of this research study, member checking and triangulation methods were utilized. The study participants had the opportunity to examine the transcripts of their interview responses to ensure accuracy and misinterpretations did not occur (Creswell, 2013). Triangulation was achieved by using and coding multiple sources of data collected from interviews and the review of documents, such as behavior, suspension and TELL Survey data. Using several sources of information helped make this collective case study more accurate and convincing (Yin, 2014).

\section{Dependability}

Dependability is the extent that other researchers could repeat a study and that the findings would be consistent. This collective case study followed identical procedures to ensure consistency among data collection and data analysis from each participant, both teachers and the principal, at an urban, low-performing elementary school.

In addition, the researcher utilized multiple sources of data to strengthen the construct validity and provide multiple measures of the same phenomenon (Yin, 2014). Triangulation of data collected from interviews, document analysis and TELL Survey data helps to strengthen the confidence in the findings obtained and provide reliability of the conclusions. 


\section{Transferability}

In qualitative research, transferability is how the researcher demonstrates that the research study's findings are applicable to other contexts, i.e., similar situations, similar populations and similar phenomena. To ensure transferability of this case study, this researcher sought to provide thick description and accuracy of the setting, participants and procedures utilized. The methods of data collection and data analysis are clearly and systematically indicated so replication of this collective case study can occur in the future.

\section{Confirmability}

Confirmability refers to the degree of neutrality in the findings of a research study. Simply stated, confirmability ensures that the study's findings are based on the participants' responses and no potential biases or personal motivations of the researcher manifest. To guarantee confirmability of the findings of this research study, this researcher can provide an audit trail which highlights the step by step coding process of data analysis to provide a rationale for the decisions made by the researcher. This data trail establishes that the research study's findings accurately portray the responses of the participants.

\section{Limitations}

In qualitative research, the researcher is the instrument of study and raises possible issues of researcher bias. This researcher limited researcher bias by intentionally focusing on the experiences of the participants without any purposeful inference in theme or pattern interpretation. A second possible limitation is the fact that although participants share the common experience of implementing SWPBIS in an urban, persistently low- 
performing elementary school, contextual factors will play a role in individual experiences because participants have different levels of teacher preparation, years of experience, experience in varying contexts, teach different students, and experience different challenges on a daily basis. In addition, this researcher is ever mindful that interviews are, by nature, self-reported accounts that speak to the perceived contextual factors that influence the opinions of participants.

This researcher is currently employed as an educational recovery leader supporting persistently low-performing schools in the state where this study is situate and has witnessed firsthand how SWPBIS frameworks function in schools in various stages of implementation. Over the past fourteen years, this scholar has worked in the field of education as a substitute and classroom teacher, instructional coach and turnaround specialist at various elementary, middle and high schools and currently work in two lowperforming schools in the school district where this research will take place.

Thus, this study took place "in this researcher's own backyard" which brought with it possible bias issues and "insider" knowledge. This researcher made a focused and sincere attempt to maintain the integrity of the study and maintain the integrity of the teachers' and principals' perceptions of their schools' SWPBIS framework without including her own voice in the results.

\section{Summary}

This study lends additional information to the current body of knowledge regarding the effectiveness of SWPBIS in public schools. Because this study was qualitative in nature, it engaged the instrument of teacher and principal voice to provide information to teachers, administrators and school district personnel to determine whether 
classroom climate and student behavioral issues at an urban, low-performing elementary school can be improved as a result of the implementation of school- wide Positive Behavior Interventions and Supports. To ascertain teacher and principal perceptions and attitudes, interviews provided rich data and focus on the classroom experiences, i.e., lived experiences, of teachers implementing SWPBIS in urban, low-performing schools in hopes of improving PBIS effectiveness to produce more positive future outcomes both school and district-wide.

This study is significant for the district in which the study takes place because it is a large, urban public school district in the Southeast Region of the United States. In 20142015, this district was compared to all school districts in its state based upon state required accountability testing results and received a classification of needs improvement. Of the nearly thirty lowest-performing schools in the state, the majority of those schools are located in this school district.

Information obtained from the website of the school district that is the subject of this study shows this school district began implementing the School-Wide Positive Behavior Interventions \& Supports (SWPBIS) framework at 53 schools in 2013-2014 and expected to obtain full implementation district-wide within 3-5 years. In 2013-2014, the designated schools engaged in one-full year of PBIS training which included resources, training from the Kentucky Center for Instructional Discipline and university personnel.

In 2014-2015, the majority of the schools receiving PBIS pilot training the previous year attemped full school-wide implementation of PBIS and additional PBIS schools were added to the district cohort. Based on the districts' implementation goals, 
the entire school district was slated to fully implement PBIS in all of its schools by the 2017-2018 school year.

At the time this study was conducted, the elementary school selected for this case study had been implementing SWPBIS for at least three years. Recall, the purpose of this study, therefore, was to discover teacher and principal perceptions of the effectiveness of School-Wide Positive Behavior Interventions and Supports to inform the pros and cons of continued use of this framework and possible modifications needed to ensure its effectiveness in urban low-performing elementary schools. Additionally, participant perceptions seek to provide in-depth information to determine if school-wide PBIS achieves its goal to create a positive "host environment" which positively impacts school culture and creates an environment where high levels of teaching and learning can take place in urban, low-performing schools. 


\section{CHAPTER IV}

\section{DATA ANALYSIS}

In this chapter, the researcher presents an in-depth look at the data analysis process and findings from this case study conducted at Dorothy Height Elementary School. First, the researcher will identify pseudonyms for each participant and provide some background information about each participant to add detail regarding years of teaching/administrative experience and the specific contexts in which the teaching took place. Next, the themes and sub-themes that emerged from the data analysis process are discussed in-depth. Finally, the results for each research question are presented and discussed through analysis and triangulation of the face-to-face interview, suspension/referral (ODRs) and TELL Survey data to identify possible implications for school and district personnel regarding future use of School-Wide Positive Behavior Interventions and Supports (SWPBIS) to improve school culture and climate.

The purpose of this collective case study was to employ qualitative research methods to examine teacher and principal concerns and perceptions of the effectiveness of SWPBIS in low performing schools in a large, urban school district in the Southeast Region of the United States. Research question 1 sought to determine how teachers and the principals of an urban, low-performing elementary school perceive the overall effectiveness of SWPBIS on decreasing student misbehavior and improving overall school climate. Research question 2 examined what teachers and the principal of an 
urban, low-performing elementary school perceive as obstacles and supports to SWPBIS implementation, sustainability and effectiveness.

The rationale for the design of this research study emanates from a lack of literature exploring teachers' and principals' perceptions of a SWPBIS framework at an urban, low-performing elementary school and its influence on school climate. As previously highlighted, Warren et al. (2003) found the unique challenges of urban schools require more preparation and training to successfully implement SWPBIS. Ample research exists on SWPBIS effectiveness in suburban and rural schools, but, insufficient research exists on SWPBIS in large, urban, public school districts where there is an increased mix of ethnic and culturally diverse students, higher rates of students living in poverty and a greater predominance of antisocial behavior school and district-wide.

\section{Participant Perceptions of Positive and Negative Behaviors}

During face-to-face interviews, teachers and the principal were also asked to provide general perceptions of the causes of positive and negative student behaviors in school. Participants shared strategies employed to support positive student behaviors, interrupt negative behaviors and prevent negative behaviors. The first seven interview questions revealed participants' basic assumptions about student behavior and provided a deeper understanding of their prior teaching experiences and the various contexts in which they taught.

This information was analyzed using first, second and third cycle coding to ultimately reveal themes which provide contextual data about how teachers synthesized their perceptions SWPBIS framework effectiveness in an urban, low performing elementary school. 
Table 1

Summary of Participant Perceptions of Positive and Negative Student Behaviors

\begin{tabular}{|c|c|c|c|}
\hline Teachers & $\begin{array}{l}\text { Urban, Suburban } \\
\text { or Rural Teaching } \\
\text { Experience }\end{array}$ & $\begin{array}{l}\text { Causes of Positive } \\
\text { Behavior }\end{array}$ & $\begin{array}{l}\text { Causes of Negative } \\
\text { Behavior }\end{array}$ \\
\hline $\begin{array}{l}\text { Wilma } \\
14^{\text {th }} \text { year }\end{array}$ & Urban, suburban & $\begin{array}{l}\text { Positive } \\
\text { reinforcement }\end{array}$ & $\begin{array}{l}\text { Inability to control } \\
\text { emotions }\end{array}$ \\
\hline $\begin{array}{l}\text { Ellen } \\
22^{\text {nd }} \text { year }\end{array}$ & Rural & $\begin{array}{l}\text { Positive } \\
\text { reinforcement }\end{array}$ & $\begin{array}{l}\text { Family life, } \\
\text { negative teachers }\end{array}$ \\
\hline $\begin{array}{l}\text { Patricia } \\
24^{\text {th }} \text { year }\end{array}$ & Urban, rural & $\begin{array}{l}\text { Positive } \\
\text { reinforcement, } \\
\text { relationships }\end{array}$ & $\begin{array}{l}\text { Lack of success, } \\
\text { negative } \\
\text { relationships } \\
\end{array}$ \\
\hline $\begin{array}{l}\text { Gayle } \\
12^{\text {th }} \text { year }\end{array}$ & Urban, rural & $\begin{array}{l}\text { Parent involvement, } \\
\text { high expectations, } \\
\text { success }\end{array}$ & $\begin{array}{l}\text { No parent } \\
\text { involvement, low } \\
\text { expectations }\end{array}$ \\
\hline $\begin{array}{l}\text { Daniel } \\
20^{\text {th }} \text { year }\end{array}$ & Urban & $\begin{array}{l}\text { Sense of belonging, } \\
\text { clean school, } \\
\text { feeling safe }\end{array}$ & $\begin{array}{l}\text { Adversarial } \\
\text { relationships, low } \\
\text { expectations }\end{array}$ \\
\hline $\begin{array}{l}\text { Marcy } \\
15^{\text {th }} \text { year }\end{array}$ & Urban, rural & $\begin{array}{l}\text { Positive } \\
\text { reinforcement, } \\
\text { relationships }\end{array}$ & $\begin{array}{l}\text { Low academic } \\
\text { skills, inability to } \\
\text { control emotions }\end{array}$ \\
\hline $\begin{array}{l}\text { Carla } \\
9^{\text {th }} \text { year }\end{array}$ & Rural & $\begin{array}{l}\text { Success, } \\
\text { relationships }\end{array}$ & $\begin{array}{l}\text { Academic struggle, } \\
\text { lack of confidence }\end{array}$ \\
\hline $\begin{array}{l}\text { Alice } \\
2^{\text {nd }} \text { year }\end{array}$ & Urban & Relationships & Copycat behavior \\
\hline $\begin{array}{l}\text { Candace } \\
20^{\text {th }} \text { year }\end{array}$ & Urban, Suburban & $\begin{array}{l}\text { Positive } \\
\text { reinforcement, } \\
\text { praise, success }\end{array}$ & $\begin{array}{l}\text { Teacher bias, lack } \\
\text { of praise and } \\
\text { feedback }\end{array}$ \\
\hline $\begin{array}{l}\text { Arnell } \\
9^{\text {th }} \text { year }\end{array}$ & Urban & $\begin{array}{l}\text { Positive } \\
\text { experiences, } \\
\text { success }\end{array}$ & $\begin{array}{l}\text { Lack of positive } \\
\text { relationships, } \\
\text { unsuccessful }\end{array}$ \\
\hline \multicolumn{4}{|l|}{ Principal } \\
\hline $\begin{array}{l}\text { Norma } \\
19^{\text {th }} \text { year }\end{array}$ & $\begin{array}{l}\text { Urban, Suburban } \\
\text { and rural }\end{array}$ & $\begin{array}{l}\text { Extrinsic } \\
\text { motivation }\end{array}$ & $\begin{array}{l}\text { Outside factors, } \\
\text { mental health issues }\end{array}$ \\
\hline
\end{tabular}


The data in Table 1 above depicts the various pre-conceived notions teachers and the principal at Dorothy Height Elementary School have about the causes of positive and negative student behaviors at their school. Participants were asked general questions not specifically related to the context of the school selected for this study. Thus, the teachers and principal drew upon their prior knowledge and teaching experiences in rural, urban and suburban contexts to explain their perceptions of why students exhibit positive and negative behaviors in school. A thematic analysis of participant responses reveals similarities in teacher perceptions of the causes of positive student behaviors.

Results of thematic analysis reveal six of the ten teachers interviewed feel positive reinforcement from the teachers results in positive student behaviors in school. This recurring response demonstrates the majority of teacher participants believe that reinforcing the positive behaviors desired from students is the best way to ensure the repetition of those behaviors. It would appear that most of the participants in this study believe that positive behaviors beget positive behaviors. Thus, it seems plausible to assume that most of the teachers would support the implementation of SWPBIS, because its framework relies on pre-empting negative students behaviors by instituting positive language, relationships, rewards and celebrations for following expectations. If this is the case, the majority of teacher participants in this study do not hold pre-conceived notions about SWPBIS that would negatively impact its implementation, sustainability and effectiveness to create a host environment for student success.

A notable dissenting opinion of why students exhibit positive behaviors in school emanates from the principal. Norma, an administrator with nineteen years of experience in the field of education, perceives that extrinsic motivators, such as rewards, are the 
main cause of positive student behaviors. It is worth mentioning that none of the other four remaining teachers share the principal's belief that extrinsic motivation causes students to exhibit positive behaviors. The lack of agreement between the majority of teachers and principal on what causes positive student behaviors is surprising and may prove relevant in the discussion of findings and conclusions.

Conversely, four of the ten teachers interviewed perceive the lack of positive relationships with teachers and negative or adversarial relationships cause students to exhibit negative behaviors. It is immediately apparent that participant responses are more varied for the causes of negative behaviors than participant perceptions of causes of positive behaviors. Three of the ten teachers feel students exhibit negative behaviors as a result of low academic skills and feeling unsuccessful in school. Two teachers believe students act out due to an inability to control their emotions and Alice, the teacher with the fewest years of experience, perceives students behave inappropriately as a form of copycat behavior. Norma, the principal, perceives that students display negative behaviors in school as a result of outside factors, such as home life, and mental health issues.

This baseline data provides a precedent to establish participants' general perceptions of why students act positively or negatively in school settings. For the purposes of this study, it is beneficial to identify participants' central beliefs about why students behave the way they do and consider if their pre-conceived notions impact the data positively or negatively. 


\section{Four Major Themes}

Four major themes developed from three cycles of coding and data review and each of these themes provides in-depth understanding for Research Question 1. The four themes derived from this data analysis include:

1. SWPBIS Ineffective/Does not Work

2. High Levels of Teacher Frustration

3. Increased evidence of Violent Student Behaviors

4. No Consequences/Students Rewarded for Inappropriate Behaviors

\section{SWPBIS Ineffective/Does Not Work}

The foundational basis, or theoretical framework for this research study, hinged upon determining whether or not School-Wide Positive Behavior Interventions \& Supports (SWPBIS) works to improve student misbehavior and overall school climate in an urban, elementary school. Based upon recurring responses to interview questions from both teachers and the principal, TELL Survey data, Office Discipline Referral (ODR) data and student suspension data, the consistent, recurring response is that SWPBIS is ineffective and doesn't work to improve overall school culture and climate and decrease inappropriate student behaviors. Participants expressed their perception that SWPBIS does not work in the following ways:

School-wide, I don't see a lot of change honestly. I hear a lot of other teachers talking. I hear students running up and down the hallways or screaming, hitting, kicking doors. I hear a lot. My classroom is pretty good. And, again, I think it's partly to do with, I already knew a lot of these students last year. I learned from mistakes I made last year, relinquishing my control to calling CRT (Crisis Response Team) and just handling it myself, I think has made a huge, huge difference. But school-wide, I haven't noticed a lot of change in a positive way. (Alice, Interview) 
I'm not sure they really have changed that much. I still see the same behaviors we've had since I've been here, maybe just less of them. We still have slamming of doors, kids walking out of class every day. We still have them throwing fits. I think one of the differences is we have lower numbers of students. When I started here, we had over 400 students, and we're down to 250 students. So, I think sometimes that makes it seem like it's a lot better, when really, I feel like with 250 kids, the behaviors are more escalated. (Candace, Interview)

I think, first of all, own it. Own it, that it's not working. We have to say.... and, you know, sometimes that hurts feelings. The social growth is different and I think that's when you tailor the program to meet the needs of your school. Because you can't blanket everybody. I think we try to blanket everybody with the same program, but you're not really looking at the academics of the program and how you could best engage students who are older and more mature and be a little bit more sneaky and conniving, you know what I mean...more intelligent about how to be bad or how to be good. (Daniel, Interview)

I think we have an awareness. I don't think that we're... I know we might be trying to make an effort; but we're not, just not successful. I can't think of any students that I truly see their behavior has changed, and stayed at an improved level for a considerable amount of time since we've implemented it. (Gayle, Interview)

According to all four participants above, SWPBIS is ineffective and has made little or no impact toward improving overall school climate. Daniel, a $5^{\text {th }}$ grade teacher, flatly states that it is not working and the teachers and principal need to own it. The quotes provided from multiple participants about perceptions of SWPBIS effectiveness and teacher and principal concerns about SWPBIS demonstrates a common theme among teachers that SWPBIS at their school is ineffective and does not work to improve overall school climate.

Surprisingly, Norma, the principal, agreed with her teachers' assessments that SWPBIS is not working to improve overall school climate for the students, staff and administrators at Dorothy Height Elementary School.

It makes it hard to say it's working because we struggle with how to give them (students) what they need and I don't know if it's because we're so hung up on 


\section{traditional school versus doing whatever it takes and thinking outside the}

box. (Norma, Principal, Interview)

To justify her response that SWPBIS in her school is ineffective, Norma identified several reasons for its ineffectiveness including a lack of formal training provided from the school district, a transient student population, teacher turnover and an increase in more physically violent behavior exhibited by students in recent years. Norma explains further:

It's non-existent. I think because of our transient population because just when we finally get more kids than not following the PBIS protocol, then summer comes, we get a whole new group of kids that have no PBIS experiences. It's like starting over when we think and feel like we should be transitioning on and the new kids change the dynamic of what we had. We always seem to get the tough kids from other schools that walk in to my tough kids and then it becomes the alpha war and one kid is trying to use PBIS strategies but the other kid doesn't know how to use coping and ignoring strategies and walk away and let it go. It goes back to outside factors. (Norma, Principal, Interview)

A triangulation of data from the 2017 TELL (Teaching, Empowering, Leading and Learning) Survey of teacher perceptions on school climate, Office Discipline Referral (ODR) data and student suspension data provide a more in-depth look at the state of the overall school climate at DHES. As previously discussed, the TELL Survey is a biennial survey that offers every certified educator in the state where this survey is conducted the opportunity to provide input on teaching conditions and school leadership at the school where they work.

These data can be used to inform school leaders when making decisions about school vision, mission, professional development, behavior policies, facilities and resources, etc., to improve overall culture and climate and student achievement. District leaders can also use this information, which is reported for each school in the district, to 
analyze patterns and trends between different school contexts within the same school district.

Every two years, TELL Survey results are available from the state department of education website is disaggregated by school, district, and state and provides invaluable insight into the analysis of teacher perceptions of student behavior in the school and overall school climate. The most recent TELL Survey was conducted in the spring of 2017 and almost $91 \%(41,500)$ of educators in the state responded. In $2017,100 \%$ of educators at DHES responded to the survey, which is an increase from an $80 \%$ educator response rate at the school in 2015. TELL Survey data provides educators and administrators with tools to facilitate continuous school improvement and includes questions on the following topics:

- Community Engagement and Support

- Teacher Leadership

- School Leadership

- Managing Student Conduct

- Use of Time

- Professional Development

- Facilities and Resources

- Instructional Practices and Support

- New Teacher Support

The table below depicts a comparison of TELL Survey data results from 2017 and 2015 for Dorothy Height Elementary School. The data shows the percentage of licensed educators who agree with each TELL Survey Indicator statement. It is important to note that Norma, the principal of DHES, has been at DHES since 2014, 
and was the principal during both TELL Surveys, allowing for a comparison of teacher perceptions under the same principal leadership.

Table 2

Dorothy Height Elementary School TELL Survey Comparison Data

\begin{tabular}{|c|c|c|}
\hline TELL Survey Indicator & $\begin{array}{l}\text { DHES } 2017 \\
\text { \% Agreement }\end{array}$ & $\begin{array}{c}\text { DHES } 2015 \\
\text { \% Agreement }\end{array}$ \\
\hline $\begin{array}{l}\text { Q5b. Students at this school follow } \\
\text { rules of conduct }\end{array}$ & $3.0 \%$ & $3.2 \%$ \\
\hline $\begin{array}{l}\text { Q5g. The faculty work in a school } \\
\text { environment that is safe }\end{array}$ & $18.2 \%$ & $29.0 \%$ \\
\hline $\begin{array}{l}\text { Q2c. Teachers are allowed to focus } \\
\text { on educating students with minimal } \\
\text { interruptions }\end{array}$ & $18.7 \%$ & $25.8 \%$ \\
\hline $\begin{array}{l}\text { Q3g. The school environment is } \\
\text { clean and well maintained }\end{array}$ & $24.2 \%$ & $56.2 \%$ \\
\hline $\begin{array}{l}\text { Q5d. School administrators } \\
\text { consistently enforce rules for } \\
\text { student conduct }\end{array}$ & $15.2 \%$ & $41.9 \%$ \\
\hline $\begin{array}{l}\text { Q7.3.g. The school leadership } \\
\text { makes a sustained effort to address } \\
\text { teacher concerns about managing } \\
\text { student conduct }\end{array}$ & $25.0 \%$ & $56.2 \%$ \\
\hline $\begin{array}{l}\text { Q10.6 Overall, my school is a } \\
\text { good place to work }\end{array}$ & $28.1 \%$ & $54.8 \%$ \\
\hline
\end{tabular}

Source: www.tellkentucky.org

Following a thorough review of the TELL Survey comparison data, it is evident from the table above that the overall school climate at DHES in 2017 has declined from the TELL Survey results from 2015. In 2015, a marginal majority percentage, $54.8 \%$ of teachers, perceived their school as a good place to work but, in 2017 , only $28.1 \%$ of teachers feel overall their school is a good place to work. Despite having the same principal, the data supports the teacher and principal interview responses, which show no 
positive improvement in school culture and climate. To the contrary, in 2017 teachers at DHES perceive the overall school culture and climate declined in the past two years. TELL Survey (2017) data appears to support teacher and principal perceptions that SWPBIS presents no positive improvement in school culture and climate and, thus is ineffective at improving the overall school culture and climate.

Moreover, 2017 TELL Survey data shows only 3\% of teachers feel that students at DHES follow school rules of conduct, which is relatively unchanged from the 2015 TELL Survey data point of $3.2 \%$. It is difficult to imagine a healthy school culture and climate in a school where only three percent of teachers feel students follow the rules of conduct. SWPBIS implementation occurred at DHES during the 2013-2014 school year, yet the percentage of teachers who feel more students are following the rules of conduct remains relatively unchanged. The question arises as to whether it is an issue of training and implementation, or, as the principal stated, outside factors that are preventing SWPBIS from making a positive impact on overall school culture and climate.

Lastly, one of the foundational purposes for this pragmatic qualitative study on teacher and principal perceptions of the effectiveness of SWPBIS in urban, lowperforming schools centers on the essential right of students to attend schools that are safe and orderly. In 2017 , only $18.2 \%$ of teachers at DHES perceived a safe working environment at their school. Unfortunately, this percentage had dropped from $29.0 \%$ in 2015, which tends to support teacher and principal perceptions that the school environment is not safe due to increasingly more violent student behavior and overall classroom disruptions. 


\section{High Levels of Teacher Frustration}

The second emergent theme derived from teacher and principal interviews entails frustration with SWPBIS' ineffectiveness to produce a safer, more orderly learning environment for teachers and students. Recall Sugai and Horner (1994) posited the ultimate goal of SWPBIS is the creation of effective "host environments" that support the use of proactive discipline policies. Interview questions 18 through 20 asked each participant to respond to questions about how they feel about SWPBIS at DHES.

Utilizing Fuller's Concern-Based-Adoption-Model (CBAM), all study participants were asked to respond to the following question to identify concerns about SWPBIS: When you think about SWPBIS, what concerns do you have?

Overwhelmingly, second cycle coding analysis produced the theme that teachers are frustrated, overwhelmed and perplexed about SWPBIS at DHES. Several participants expressed their frustration with SWPBIS in the following statements:

I think everybody is incredibly frustrated. I think the morale is low and they still don't feel like they are getting what needs to be done, done. And what I mean by that is having someplace safe for these extreme behaviors to go... they don't have that and so they're still feeling like they're teaching nothing. I've heard teachers say "I've taught four equations in one class all day because of those behavioral issues." And there is only so much I think they can take. I think emotionally, it's just very draining. (Wilma, Interview)

The teachers feel frustrated. I mean, they feel isolated and they don't feel supported, and it goes back to the consequences. The teachers feel like they can be hit on, called names and disrespected and it's "Oh well, that's just where you work." (Gayle, Interview)

Teachers are frustrated because a lot of behaviors that we are dealing with, aren't dealt with. So they're just ongoing and it's mostly, most of it is the same group of kids and the same behaviors day in and day out that are never really addressed but always pacified. So, they're not being dealt with, whether proactive or reactive. (Arnell, Interview) 
We have a lot of new teachers and I think sometimes they may not know different ways they can de-escalate behavior or provide that positive support... and they get frustrated. So, for me, I don't feel like it's the most effective plan that I guess we could have. (Candace, Interview)

Frustration is a major concern shared by the four participants above and, based upon the emotion in their statements, this researcher would argue, poses a significantly negative impact on efforts to create a healthy school culture and climate. Student success in school hinges upon the most important variable, the teacher, having a positive outlook on the teaching and learning environment and the ability to teach with minimal student disruptions. As stated above, teachers are frustrated because they are being assaulted and disrespected while attempting to teach the curriculum, and students are not receiving consequences for inappropriate behaviors.

The 2017 TELL Survey data, as referenced in Table 2 above, indicates only $18.7 \%$ of teachers feel they are able to focus on educating students with minimal disruptions. This data would help to explain some of the teachers' frustration with student behaviors that continually disrupt instruction and, at times, cause teachers to feel like they can be assaulted and disrespected on a daily basis with little or no consequences for misbehaving students. Office Discipline Referral (ODR) Data at Dorothy Height Elementary School was analyzed through a document review process in an effort to triangulate the interview and TELL Survey data to determine if patterns or trends exist to support participant perceptions.

At DHES, teachers complete discipline referrals, known as Behavior Intervention Logs (BILs) every time a call is made to the Crisis Response Team (CRT) to assist with student misbehavior in the classroom. A school-wide behavior policy is in place which utilizes a behavior intensity chart that provides teachers with specific protocols about 
what types of behaviors warrant CRT calls for assistance and the various levels of intensity 1-5. Based upon this chart, teachers determine when a CRT call is appropriate and if an immediate response is needed to ensure the safety of students and staff. DHES' behavior intensity chart appears in Appendix C.

A review of the DHES Behavior Intensity Chart suggests that students may repeatedly exhibit a litany of misbehaviors prior to reaching Intensity level 3, which warrants a CRT call, only after the teacher has attempted three classroom level interventions to re-direct student behavior. Teachers are expected to ignore and re-direct many Level 1 and Level 2 behaviors, using SWPBIS strategies of re-stating expectations and reward systems, but if students continue the disruptive behaviors, it makes it extremely difficult for teachers to deliver high-quality instruction. Further, teachers are expected to complete a Behavior Intervention Log (BIL) each time a CRT member is called to remove a student from the classroom, and this creates additional administrative paperwork for the teacher that takes time away from lesson plan periods used to plan instruction, grade assessments, contact parents, etc.

Thus, the theme of teacher frustration with SWPBIS effectiveness at DHES, or lack thereof, could be construed as a realistic perception on the part of teachers expected to teach despite ongoing disruptions, disrespect, and often, unsafe and volatile environments. From a pragmatic point of view, it is apparent that teacher frustration is an example of SWPBIS not working to improve student misbehavior and overall school climate. 


\section{Increase in Evidence of Violent Student Behaviors}

From the third cycle coding and thematic analysis of the participant interviews, a third theme emerged. Within-case and cross-case analysis showed both teachers and the principal at DHES share concerns that, since the implementation of SWPBIS, student behaviors are increasingly becoming more violent. Student behavior in public schools across the United States is increasingly growing more violent and negatively impacts school culture.

Researchers have found that violence in schools negatively impacts student academic achievement and behavior, adversely affects teacher and staff job performance and impairs teacher and parent relationships (Daniels, Bradley \& Hays, 2007). Study participants expressed concerns about the school environment being unsafe and that the lack of consequences for inappropriate and violent student behavior is hurting students. Additionally, participants shared concerns that administrators are not addressing dangerous children and their mental health needs.

In response the interview question 21 about SWPBIS concerns, multiple teachers and the principal shared serious concerns about increasingly violent student behaviors at DHES. Teacher and principal responses were coded into recurring themes and specific statements that illuminate participant concerns regarding violent behaviors are found below:

We have way too many physically dangerous children in this school and we're not addressing those children's needs. Those children with the most extreme behavior are going to, if they get a suspension, then it's for one or two days and very few of them are getting the kind of mental health support that they need. (Wilma, Interview)

Well, more than anything, I have to say big concerns. We aren't making our classrooms safe. We're really hurting a lot of students because they're not 
getting instruction because they are having to wait while we just kind of juggle negative behaviors. (Patricia, Interview)

School-wide, there's just a lot of chaos, a lot of kids running in and out. Certain kids will run the school and you honestly, at times, can feel like you're in a mental hospital with the screaming...the hitting. You're not even talking about a temper tantrum or a meltdown. It's beyond anything I've ever seen honestly. (Alice, Interview)

Norma, the school principal, acknowledged similar concerns about the increase of more physically violent student behaviors but also stated that she has seen some improvement in the time it takes to de-escalate students. Her concerns appear below:

I think the behavior has gotten more physical, but I think it has improved in different ways as far as how long it takes us to de-escalate. It doesn't take us hours to de-escalate most kids whereas before it was taking us 45 minutes to an hour to even have a conversation with the student. (Norma, Principal, Interview)

None of teacher participants perceived improvement in student behaviors at DHES. The principal's statement above acknowledged student behaviors had become more physical. Her statement about the time it takes to de-escalate students exhibiting violent behaviors actually highlights the severity of the student behaviors at DHES which requires teachers, administrators and CRT members to respond to calls quickly to ensure the safety of the student, other students and the school staff.

A review of the Office Discipline Referral data (BILs) at DHES for the past two years provides additional information about the total number of behavior incidents reported that require CRT intervention. Additionally, suspension data from the school district data management center was reviewed to determine increases or decreases in student suspensions for inappropriate behaviors over the past five years. 
These data provide triangulation of the teacher and principal perception data to determine the overall of effectiveness of SWPBIS to improve the overall climate at DHES. Table 3 below reports the number of BILs or Office Discipline Referrals completed by teachers at DHES for the past two years.

Table 3

Dorothy Height Elementary School Discipline Referral Trend Data

\begin{tabular}{|l|l|l|}
\hline Month & $\mathbf{2 0 1 7 - 2 0 1 8}$ & $\mathbf{2 0 1 6 - 2 0 1 7}$ \\
\hline August & 58 & 207 \\
\hline September & 330 & 664 \\
\hline October & 447 & 513 \\
\hline November & 531 & 442 \\
\hline December & 220 & 282 \\
\hline January & 195 & 382 \\
\hline February & 209 & 381 \\
\hline & & \\
\hline Total & 1,990 & 2,871 \\
\hline
\end{tabular}

An examination of the table above indicates that BILs have decreased approximately $31 \%$ overall from 2,871 to 1,990 through the end of February 2018. A review of the behavior policy at DHES shows that the procedures for when teachers should call CRT has been changed in 2017-2018 to reflect that teachers must attempt to re-direct student misbehaviors in the classroom at least three times prior to calling for CRT support. Therefore, the data purports a decrease in discipline referrals, but it is unclear if students are displaying fewer numbers of inappropriate behaviors or if teachers 
are being instructed by administrators not to call for behaviors deemed less severe. Based on teacher perception data, incidences of student disruptions and increasingly more violent, unsafe behaviors have not decreased.

One other possible explanation for the appearance of decreased CRT call volume is the drop in student enrollment from approximately 304 students in 2016-2017 to approximately 260 students in 2017-2018. A loss of forty students could make a significant impact on the number of BILs and calls for CRT assistance, thus creating the effect of fewer issues of violent student behaviors when, in fact, teacher perceptions are that they have actually increased despite having fewer students enrolled. Nonetheless, perception is reality and, for the purposes of this case study, teacher and principal perceptions provide extensive insight into their view that SWPBIS is ineffective because student behavior is becoming more physically violent and threatens efforts to create a safe and positive learning environment. In short, if physically dangerous children are making the school unsafe on a daily basis, teachers, staff and administrators are less likely to perceive SWPBIS is effective.

\section{No Consequences/Students Rewarded for Inappropriate Behaviors}

The fourth emergent theme is teachers at DHES perceive students receive no consequences for inappropriate behaviors and are actually rewarded when they exhibit negative behaviors in the classroom, hallways, lunchroom, playground and in special area classes. In 2016, 3.2 million students in the United States received out-of-school suspensions for behaviors such as cursing, bullying, defiance, horseplay, rule violation, cutting class, sexual harassment, fighting and vandalism (NCES, 2016). Based upon this statistic, it is evident that students are exhibiting more serious disruptive behaviors in 
school and many teachers share the perception that students aren't held accountable for their actions.

Contrary to popular belief, SWPBIS is not a program, but a systems approach to creating the social culture and behavior supports necessary for all students in a school to achieve both social and academic success. The goal is to create a school wide environment that utilizes a proactive approach to discipline to prevent student misbehaviors. The SWPBIS framework also promotes celebrating students who regularly exhibit appropriate behaviors in a positive way. As part of this goal, DHES students are often provided with opportunities to earn tickets for good behavior or receive Class Dojo points for good behavior to spend at the classroom or school store or earn classroom celebrations such as a popcorn party.

Unfortunately, the teachers at DHES share the perception that students who follow school rules are not celebrated or rewarded and, conversely, disruptive students receive rewards for acting out in class and continue to make bad choices because they know they will receive a reward if they turn their behavior around, or at least pretend to do so. To make matters worse, teachers perceive that administrators (Principal, Assistant Principal, and Counselor) do not enforce consequences for inappropriate and disrespectful behaviors toward other students, teachers and support staff.

Teachers are concerned that misbehaving students regularly receive rewards, food or candy after making poor behavior choices and administrators do not follow-through with the school behavior policy and/or the student code of conduct policy of the school district. A few examples of what teachers perceive as a lack of consequences for misbehavior and students receiving rewards for inappropriate behaviors are shared below: 
I feel that in our school, we give too much attention to the negative behaviors. Children can leave the classroom and be gone for hours and it's unexpected for them to be back. I think excuses are made for some our kids (lack of medicine, etc.) and I understand that we need to be more patient in those things, but we also don't need to let them run the school. I have had kids gone for hours at a time and I have walked in there and they are playing with pillows. (Gayle, Interview)

Tangible objects are not effective because they need to learn to stay in the classroom to learn and be a productive citizen in society, not so you can get Skittles or whatever the bribery is. And I'm just as guilty as anybody else. (Marcy, Interview)

Some children who have a documented record of negative behavior need to have alternatives because they haven't been able to demonstrate control of their behavior and threatens the other kids so it's a physical harm. That child needs to have a different intervention because taking them out for five minutes and then coming back with "Hot Fries" or a pencil isn't getting it. (Wilma, Interview)

We are kind of pacifying negative behaviors. A lot of times students are rewarded. Toward the end of the day, they're rewarded for making their point system, and they actually had an absolutely horrible day. So, bottom line, when a child knows, "I will get a reward if I behave in that manner" and we see that a lot. (Patricia, Interview)

This mindset of giving rewards for misbehavior and a lack of consequences appeared in the principal's response to interview question 1: What are some things that cause students to exhibit positive behavior in school? Apparently, Norma believes that students are motivated by extrinsic rewards to follow classroom and school rules. The principal is instrumental in the creation of school-wide behavior policy and her belief that students needs to receive some type of reward for making positive choices could be contributing to teacher concerns about unjust rewards and a lack of consequences. Below is an excerpt from her perception of what causes students to exhibit positive behaviors in school:

Typically, it's some kind of extrinsic type of motivator. Meaning they get something for something, for doing something. Our population struggles with 
intrinsic motivation so right now our experience has been the extrinsic keeps them motivated to do the positive things. (Norma, Principal, Interview)

As a result of the principal's perception that students are motivated by extrinsic

rewards, teacher concerns about SWPBIS at DHES are elevated to the point where some participant statements were extremely blunt. When asked how she thinks teachers at DHES feel about SWPBIS at their school, one teacher responded:

It sucks. It does. It bothers them when these kids are getting suckers or "Hot Fries" or "Doritos" or whatever when they have shown themselves in the classroom and then they go into another room where there's an adult and they've given them that. When you're turning cartwheels down the hallway and you're not in your room $90 \%$ of the time during a school day, I got a problem with that. (Ellen, Interview)

A review of suspension data for the past two school years provided an additional data point for triangulation of the theme that students do not receive consequences for violating classroom and school behavior rules. As previously discussed, Behavior Intervention Logs or Office Discipline Referrals have decreased by about $31 \%$ through the end of February of the 2017-2018 school year. Despite the decrease, teachers perceive students are not receiving consequences for repeatedly violating classroom expectations and school rules. Table 5 below shows the trend data for suspensions at Dorothy Height Elementary School for the past six school years.

Table 4

Dorothy Height Elementary School Suspensions Trend Data

\begin{tabular}{|c|c|c|c|l|l|}
\hline $\mathbf{2 0 1 2 - 2 0 1 3}$ & 2013-2014 & $\mathbf{2 0 1 4 - 2 0 1 5}$ & $\mathbf{2 0 1 5 - 2 0 1 6}$ & $\mathbf{2 0 1 6 - 2 0 1 7}$ & $\mathbf{2 0 1 7 - 2 0 1 8}$ \\
\hline 21 & 83 & 123 & 132 & 104 & 130 \\
\hline
\end{tabular}

Source: Data Management Center, MCSD

Through the end of February 2018, DHES teachers completed 1,990 BILs/ODRs. Table 4 shows, through the end of February 2018, DHES administrators authorized 130 
suspensions, which shows only $15.3 \%$ of the office referrals were resolved through suspension. The DHES Behavior Intensity Chart shown in Table 2 depicts how teachers are to resolve student behavior issues and are only instructed to call CRT and complete an office referral (BIL) for Level 3, 4 and 5 behaviors. Behaviors in these categories include hitting, fighting, weapons and behaviors that could cause serious injury to students and staff.

Thus, the small percentage of students suspended for displaying Level 3 or higher behaviors may support teacher perceptions that students are not receiving consequences for behaviors that are physically dangerous and may actually receive rewards from administrators and other teachers to entice them to turn their behavior around.

\section{Participant Perceptions of Overall SWPBIS Effectiveness}

This researcher's interpretation of findings for Research Question 1 views participants' concerns about SWPBIS at their school as open and honest with a sincere desire for changes to improve the culture and climate of the school. The four themes: SWPBIS Ineffective/Does Not Work, Frustrated Teachers, More Violent Student Behaviors and No Consequences/Students Rewarded for Inappropriate Behaviors were evident within each and in cross-case analysis via second cycle coding and thematic analysis. Of the four themes, one could argue that the primary concern of teachers at DHES is that SWPBIS is ineffective and, in the pragmatic theoretical framework, simply does not work at Dorothy Height Elementary School. The theme of ineffectiveness may serve as the umbrella under which all of the other themes fall because the remaining themes explain in-depth reasons why SPWIS is ineffective and does not work. 
Findings suggest the Concern-Based-Adoption-Model (CBAM) used by Fuller (1969) to ascertain changes in pre-service teacher perceptions of the teaching profession before and after beginning their teaching careers. Using this method, the CBAM provided rich, thick data from the participants because the open-ended questions about SWPBIS concerns allowed an open forum to share thoughts, perceptions, feeling and unsettling concerns about the current state of behavior and culture and climate at DHES. Purposefully sampling teachers and an administrator with two or more years of experience with SWPBIS at DHES provided an opportunity for participants to compare perceptions from previous years to the current year.

Many teachers responded with concerns about teacher frustration citing escalating student misbehavior from previous years and attempts to address it through positive teaching and re-teaching of behavioral expectations, i.e., SWPBIS, remain ineffective toward creating the safe and positive school environment all students and staff deserve. Overall, teacher participants raised serious concerns about SWPBIS at DHES and thematic analysis of interview data suggested similar explanations for its ineffectiveness, i.e., emergent themes. In addition, specific participant reflections on supports and obstacles to SWPBIS effectiveness at DHES are presented.

\section{SWPBIS Obstacles}

1. Urban, High Poverty Students

2. Inconsistency/Lacks Follow-Through

3. Lack of Training for High Need Trauma Students 


\section{Urban, High Poverty Students}

The first theme that emerged as an obstacle to the implementation, sustainability and overall effectiveness of SWPBIS is working with students from urban, high-poverty neighborhoods. The effectiveness of any school-wide program or framework depends heavily on the quality of its implementation and Dorothy Height Elementary School is located in a high poverty, high crime, and urban neighborhood. Regarding SWPBIS, research has shown that urban schools require more groundwork and preparation prior to implementation to ensure the best possible effectiveness results. In the field of education, the essence of successful groundwork is teacher training.

In an attempt to answer Research Question 2 of this study, participants were asked to describe the extent of the training previously received in PBIS. Six of the teacher participants stated they had received ongoing, extensive training in other school districts where they were formerly employed. The remaining participants had only received trainings provided by MCSD personnel and the behavior coach at DHES during the summer prior to the 2016-2017 school year and embedded professional development. The principal emphatically stated that she only received one year of formal training from the school district while DHES' identification as a pilot school for PBIS in 2014-2015.

Unfortunately, the majority of teachers interviewed did not receive formal PBIS

training from the school district prior to employment at DHES and the expectation to implement SWPBIS with fidelity. Several teachers expressed the difficulties with highpoverty environments:

Personally, I don't really feel it's effective in a school like this, with our students who have high levels of trauma and poverty. I feel like they need more social and emotional support. They need to go back to building that positive school and classroom climate and culture and those relationships with their 
teacher, with other students. I think we have a lot of students how don't know how to handle conflict that could be something really simple, like "Hey, you took my pencil." Whereas I would handle that in a different way than some of our students. It would cause a knockdown, drag out fight because they don't have the skills to deal with that. (Candace, Interview)

I think for some students it works, but maybe not for all students. I think it might take longer for some what we would call our "high flyers" just because there's so many more walls to break down. Our "hot students" can be set off at just about just moment. They are a little unpredictable. They need more than just an education. They need to feel valued. They need love and they have so many more stressors than your typical child. (Carla, Interview)

Carla continued with her discussion of the additional stressors that high poverty, urban students struggle with and shared some of the most powerful insights into the challenges of SWPBIS effectiveness in this environment.

It's really hard for these kids due to the amount of home life uncertainty in regards to poverty. Sometimes we don't know if our students have had enough to eat or if they had running water or a home so those types of situations are not your typical situations for a student and not knowing if they had a bed to sleep in the night before. We deal with a lot more emotional issues than we do academic. We have to hit the emotional before we can hit the academic. (Carla, Interview)

Some teachers have never been in a climate like this so they don't know what to do as far as the children's backgrounds. This is not a traditional school far as I say "Sit down" and the student sits down. It just doesn't happen. (Ellen, Interview)

If some kids aren't responsive to the positive and are unresponsive to you being proactive, they don't like to be loved on, they don't like to be hugged because that's not what they're used to. So some of those proactive things that we do, like giving them praise, they don't respond to that. (Arnell, Interview)

The teacher interviews highlighted above provide an in-depth look into their perceptions that an obstacle to SWPBIS effectiveness is working with students from urban, high poverty schools. Candace specifically states that SWPBIS is not effective in high poverty schools like DHES because the students are victims of trauma and require 
more social and emotional supports in other to learn strategies to control their emotions and impulsive behaviors. Carla echoes Candace's opinion that SWPBIS works for some students and not for other students because students at DHES have more stressors than a typical child. She goes on to refer to certain students at "hot" and "high flyers" who have many more walls to break down and can be set off at any moment.

Ellen perceives some of the teachers at DHES are struggling with student behavior due to lack of cultural competency training and experience. Specifically, the teachers, who are majority Caucasian, don't have experience working in a school where the majority of students (89\%) are African American and living in a high crime, high poverty urban neighborhood. Ellen feels these teachers simply don't know what to do because the students at DHES do not follow teacher instructions as well as students in a more traditional school.

Arnell provides more insight into the perception that urban, high poverty students respond differently to proactive relationships and positive praise. She states that students from impoverished, traumatic backgrounds do not respond appropriately because they are not accustomed to receiving positive attention. Ultimately, the thematic analysis of the teacher interview data reveals the perception that the challenges of urban, high poverty student behaviors create a formidable obstacle to the successful implementation, sustainability and overall effectiveness of SWPBIS.

\section{Inconsistency/Lacks Follow-Through}

Inconsistency in implementation emerged as a second obstacle to SWPBIS effectiveness at Height Elementary School. Research shows the effective implementation of SWPBIS requires a three-tiered approach to create a school-wide, universal behavior 
plan to support all students and provide targeted interventions for students who need additional assistance (Sugai \& Horner, 2002). Recall from chapter two, the premise of SWPBIS is that Tier 1 school wide implementation will address approximately $80 \%$ of behavioral issues in a school, and with consistent implementation, promotes a school culture and climate that fosters successful implementation of Tier 2 and Tier 3 (Sugai \& Horner, 2002).

In the current study, interview question 20 asked participants to respond to the following question: What is needed to make the behavior and discipline practices in this school work better? Through an analysis of interview responses, several teachers believed that inconsistency in implementation of SWPBIS makes it difficult to realize the goals associated with the creation of a positive, proactive environment to support student success at school. Regardless of the universal behavior plan adopted by all school stakeholders, it is of utmost importance that the plan is actively practiced and supported by all administrators, teachers and staff.

Interview data suggest teachers do not perceive consistency in implementation of SWPBIS from all teachers in the building. Based on participant statements, it appears that teachers at DHES feel that most teachers are doing the best they can in an extremely difficult situation. More than anything, they expressed concern and disappointment with the principal's lack of consistency with leading the implementation of the school's Tier 1 behavior plan, her indecisiveness to apply consequences for inappropriate behaviors and lack of follow-through in support of teacher efforts to gain control of the school environment. Below are interview excerpts of teacher perceptions of the inconsistency of SWPBIS implementation at DHES: 
Consistency. I hear about everybody has their favorites, and you can see that. You can see that certain students, the administration treats quite differently. I've seen students who were in an all day suspension for being in a pretty violent fight in the principal's office eating pizza. I've had students brought to me by administration and told that I have a visitor or helper for the day and I find out later that helper was in fact in in-school suspension. The teacher tells me what that student had done the day before, after I have catered to them all day and made them feel like a helper 'cause that's what I was told they were. If that's what an in-school suspension is, then it's no wonder that we have the perpetual cycle of repeat offenders, which we do. (Alice, Interview)

Consistency. To consistently implement expectations, to teach them, to re-teach them. Consistency in classroom consequences, consistency in the school-wide consequences. A plan that after ten minutes, if they're not calmed down and back into the classroom, they may need to be doing this. Not just sitting until they're ready to go back to class. There needs to be some non-negotiables for the teachers, students, for administration across the board and they have to be backed up. (Gayle, Interview)

It's not consistent throughout the school. There is spurts where it's being done in our school and there are spurts when it's not. So, it's not consistent, it's not something that's in the forefront of every teacher's mind or every adult's mind in the building at all times. (Arnell, Interview)

Consistency in everyone doing the system. If you're going to put the system in place, then let's all do it. I feel like it needs to be shown to everybody. Here's the other thing, I know this population is hard to work with and there is a lot of teacher turnover, but I don't feel like it was shown to everyone here, as far as what needs to happen and how it needs to happen consistently. (Ellen, Interview)

As noted above, a theme of inconsistency developed due to multiple teacher perceptions that all teachers in building were not "doing" SWPBIS consistently and the principal was inconsistent in following through with the school-wide behavior plan and/or the school district code of conduct and intervention policy. Alice, who is completing her $2^{\text {nd }}$ year of teaching, eludes to the principal showing favoritism to certain students. While Gayle, a 12 year teacher, calls for non-negotiables for teachers, students and administrators to ensure behavior policies are enforced consistently across the board. 
In addition, Arnell, a reading interventionist who works in classrooms across the building, articulates her perception that SWPBIS is present at DHES in spurts because it is not in the forefront of every adult's mind at all times. This is especially problematic and rings true because her frame of reference includes all classrooms in the building and makes a strong statement to support the theme that a lack of consistency is an obstacle to SWPBIS effectiveness. Ellen, a math interventionist with 22 years of experience in both urban and rural schools, shares the perception that SWPBIS implementation is inconsistent. She acknowledges multiple obstacles such as; a difficult population to work with and inconsistent teacher training both contribute to a lack of overall PBIS effectiveness school wide.

Norma, the principal, acknowledges inconsistency is an obstacle to effective SWPBIS implementation, sustainability and effectiveness, but, with one caveat. She asserts several justifications for the lack of consistency and follow-through, most of which place the onus of responsibility not on herself, but on outside variables, teachers and staff. Norma's perceptions of the lack of consistency and follow-through with SWPBIS are as follows:

I think consistency. Being able to retain the staff you've invested in after you've spent a year investing and then you've got 53\% turnover rate so you're starting all over. Being cognizant of that, even the transient population of your school. I think if we had that continuity, that we would be able to see and retain the positive momentum, but I think it's hard when you can't retain. You're always going to have that teacher that's complacent whether you're a super strong school or super low school, but consistently overall being able to have a staff and a group of kids and see it all the way though, that's my ideal. That's not real, it's not real. But that's what I want for my school. I think they deserve it. (Norma, Principal, Interview) 
When prompted to explain further why Norma thinks her goal for consistent implementation is not real she stated a lack of consistent supports and belief from the community and the board of education to see that DHES truly needs a paradigm shift in its thinking about how to improve the school environment. Norma continues:

I don't think we have the supports or the belief in the community or within our board to see that our school truly needs to change the way we do school in order to do what's best for kids to ensure that all kids are learning. I think we have to think outside the box because of what our school looks like and I don't think that people are ready to own that and it's at the expense of kids.

(Principal, Interview)

Although the principal agrees with teachers on issues of inconsistency, she cites the need for a mindset shift to do what is best for the kids served by Dorothy Height Elementary School. Unfortunately, it is apparent that she is reluctant to acknowledge her role in the school's lack of consistency with SWPBIS implementation which negatively impacts sustainability of the program components to improve overall effectiveness of school wide behavior plans. In my opinion, all of these factors intersect and play a role in the consistent application of the PBIS framework. But, the building leader has the ultimate responsibility to ensure consistent SWPBIS implementation with fidelity despite factors outside of his or her locus of control. Teacher perceptions that SWPBIS is not at the forefront of adult thinking at DHES all day and every day is an obstacle to effectiveness and, regrettably, is a pervasive theme amongst teachers and the principal.

\section{Lack of Training for High Need, Trauma Students}

The third obstacle to the effectiveness of school-wide Positive Behavior Interventions \& Supports at DHES is the perception that SWPBIS does not provide the training necessary for teachers to deal with high-need students who have been exposed to 
high incidences of trauma. Often, children living in high poverty urban neighborhoods are at increased risk to exhibit antisocial behaviors due to exposure to physical and sexual abuse, family members addicted to drugs and alcohol and stress and trauma from living in high crime neighborhoods (McCurdy, Mannella \& Eldridge, 2003). Unfortunately, these disadvantaged students are ill-prepared for the school learning environment and expectations. This lack of social skills and the display of antisocial behaviors due to trauma creates a dilemma for teachers who are contracted to primarily focus on academics but cannot because they are forced to teach social and emotional strategies first before any significant content learning can occur.

Too often, due to the disruptions of students exposed to trauma, all student learning is impeded and creates a disorderly environment lacking high quality teaching and learning. These issues are palpable through observations in any classroom at DHES and teachers identified a lack of targeted training to deal with students with high need due to exposure to trauma as an obstacle to SWPBIS implementation, sustainability and effectiveness. The teacher interview quotes below provide insight into the lack of training for high need students with trauma:

The high poverty, the traumatic experiences. We have students that come in that have just experienced maybe, a family member being shot, but they're supposed to come to school that next day and function when they're still worrying about what's happening at home. We have students that frequently get CPS visits, being told they're being removed from the home. They don't know where they're going home that night. They don't know if they have food to eat. They're busy worrying about those things, so I feel like we should go more to a different model, with some more trauma-informed training. And we need to spend a good portion of our day working on the social and emotional part of our students.

(Candace, Interview)

Candace, the Goal Clarity Coach at DHES, has 20 years of teaching experience in two states in urban and suburban contexts. She believes more training is needed to better 
service students with trauma and less training with PBIS because PBIS does not meet kids where they are and provide the additional high needs trauma training needed. Wilma shares the perception that PBIS alone does not meet the needs of high trauma students and teachers need supplemental training in strategies to help children who have experienced adverse childhood experiences to respond calmly to challenging situations.

Wilma elaborates as follows:

We're setting students up for failure by not looking at their past history. We need to have something that is an immediate consequence, whether that's inschool suspension or... using our therapists or I don't know. We need to come up with something better than what we've got and data needs to guide that information. We've got hot, hot kids, the ones that are gonna be stabbing other kids with pencils that simple cannot control themselves to be able to be in a situation. (Wilma, Interview)

Both Candace and Wilma perceive violent student behaviors put the safety of all students and staff in danger and must be addressed through trauma-informed training. The persistence of high need, impulsive and violent student behaviors are a common theme that presented in various participant responses to multiple interview questions. Norma, the principal, conveyed the impression that much of the behaviors teachers are expected to deal with come from outside influences resulting from the high incidences of crime in the community, and student exposure to trauma from family members and the community. The principal shared her beliefs on SWPBIS' lack of response to student trauma and mental health needs below:

There is a lack of training (PBIS) because now we're using PATHS. Our systems evolve around the PBIS theory, but it's not PBIS. We do trauma training to try to help build a knowledge base of our staff about how to deal with our population of students and families. (Norma, Principal, Interview) 
Norma explained further that PATHS is a tier 1 social emotional learning program that targets students who need strategies to control emotions and react less impulsively to traumatic triggers. This program has been implemented since the beginning of the 20172018 school year and teachers attended mandatory training in the summer prior to the start of school. She states that she has felt for some time that SWPBIS does not address the high trauma needs of students at DHES and she used School Improvement Grant funds to provide the training materials and teacher professional development sessions. Students who are unfortunate victims of traumatic experiences need additional supports to act appropriately in school and teachers must be trained to teach students cope and self-regulate their emotions.

Teacher participants and the principal perceive the PBIS framework lacks training and supports for high need students and is an obstacle to the creation of a safe, positive learning environment at DHES. Hopefully, these same participants will continue to share concerns about the existence of this obstacle and the principal will continue to actively monitor the PATHS program and implement additional solutions to improve student behavior. On a positive note, study participants were able to identify some practices that support SWPBIS implementation, sustainability and effectiveness.

\section{SWPBIS Supports}

\section{Common Language}

2. Expectations

\section{Common Language}

School-wide common language emerged as a theme among teachers as a supporting factor for SWPBIS effectiveness. The PBIS framework posits school 
administrators, teachers and support staff must share a common language to ensure all students understand behavioral rules and understand school wide goals and expectations. Teacher and principal participants in this case study responded to the following question: What are some ways SWPBIS works to prevent negative behaviors in your classroom/school? The purpose of asking this question was to determine what types of SWPBIS elements participants perceive as supports in creating an effective SWPBIS system at DHES. Based upon their responses, having a common language used throughout the school building by administrators, teachers and support staff supports the effectiveness of SWPBIS at DHES. Below are some statements from teachers who feel that having a common language supports the effectiveness of SWPBIS at Dorothy Height Elementary School:

All the common language with PAWS throughout the hallways - positive attitude, act responsibly, wise choices and safety first. That helps in the classroom and outside the classroom because it gives all the teachers a common language. (Gayle, Interview)

PBIS empowers the teacher more than anybody because sometimes a teacher can be helpless, especially a novice or first year teacher who doesn't have a bag of tools or tricks to re-engage students. It's an advantage when they are able to go in and use strategies that maybe a student has heard before. That begins your level of trust and reinforces it, I think. (Daniel, Interview)

The two teachers highlighted above are experienced teachers who perceive some positive attributes of SWPBIS exist at DHES. The common language referred to by Gayle (pseudonym) is an acronym, PAWS, posted in the hallways, classrooms, cafeteria and restrooms at Height Elementary school. The purpose of PAWS aligns with SWPBIS objectives to create a common school wide language used by all teachers and students to project positive, proactive behaviors that promote a safe and welcoming school environment. The behavior coach ensures that PAWS language is included in the DHES 
behavior handbook and teachers receive posters to place inside their classrooms prior to the first day of school. From day one, this language exists throughout the school and the principal reinforces it on the morning announcements each day.

\section{Expectations}

One of the fundamental tenets of SWPBIS effectiveness is that all school personnel invest in systems to ensure those practices are implemented with fidelity and sustained over time (Simonsen, Sugai \& Negron, 2008). Thus, it is essential that schools attempting to implement and sustain SWPBIS policies and practices share a laser like focus on teaching and re-teaching expectations. Teacher and administrator buy-in can be an obstacle in successful SWPBIS implementation, sustainability and effectiveness, but teachers and the principal at DHES share the perception that common expectations exist and are reinforced daily.

Expectations may be construed as synonymous with having a common language for SWPBIS, but, based upon the nature of participant responses that make specific references to expectations, this researcher opted to highlight their responses separately.

Several teacher interviews below demonstrate perceptions that common expectations for SWPBIS are present at DHES and support its effectiveness:

I think it's the expectations. Establishing those expectations early and often and reminding them constantly. (Wilma, Interview)

SWPBIS tries to set up, basically, a vertical alignment of expectations, like with PAWS. So, there are expectations everywhere. (Gayle, Interview)

SWPBIS can work by helping the students create that intrinsic desire to do well by consistently reminding them, making the standards clear, going over what's good, giving them approval. But I think it has to be done consistently and it has to be meaningful. They have to know you really mean it.

(Patricia, Interview) 
Above, Patricia makes the statement that having consistent expectations for student behavior can work to help students accept the positive, proactive SWPBIS framework. She is a 27-year educator with a wealth of experience with PBIS in urban and rural school districts and believes it can work at DHES if expectations are done consistently and in meaningful ways. Lack of consistency with SWPBIS expectations will be addressed in a later discussion about obstacles to SWPBIS effectiveness.

From the principal interview, it appears that the administrators and teachers at DHES share the perception that having a common language supports the effectiveness of SWPBIS implementation and effectiveness. Norma, the principal, has been at Height Elementary School for the past five years and, in her interview, expressed a great deal of frustration with the lack of district training after the initial pilot year. Numerous times she reiterated that, after the first official district training, she and her staff have been providing their own in-house trainings based upon what she has learned on her own. Norma perceives SWPBIS common language is in place at DHES, but raises questions about the level to which students internalize the language. Her interpretation follows below:

We have a skeleton of PBIS in our building right now. The kids know what all of that stands for (PAWS), but do they internalize what that is in practice? That is the missing piece. So, we are starting to add our own pieces to it, CHAMPS, PATHS, so is it even PBIS anymore? (Norma, Principal, Interview)

While the principal acknowledges all students know the common language, she questions whether or not they really know what it looks and sounds like in practice. Participants had difficulty identifying specific characteristics of SWPBIS believed to support its effectiveness in the building. Appendix C shows the participant response 
codes for supports and it is evident that only a few responses provided synonymous codes and patterns to create the two themes identified as supports for SWPBIS. This lack of consistency in responses from study participants demonstrates that very few elements of SWPBIS are being implemented effectively and with consistency.

Norma readily admits that she doesn't know if she can officially consider the "skeleton" of SWPBIS in her building to be PBIS anymore because she has had to add additional programs to it each year in hopes of improving student behavior. But, if nothing less, DHES teachers and the principal still perceive there is a semblance of common SWPBIS language evident in the school building that provides a positive support to its effectiveness in the school.

\section{Participant Perceptions of Obstacles and Supports to SWPBIS Effectiveness}

This researcher's interpretation of findings for Research Question 2 reveals both teachers and the principal at DHES share concerns about SWPBIS effectiveness and can easily identify obstacles and barriers to the successful implementation, sustainability and overall improvement of school culture and climate. Obstacles include: urban/high poverty students, inconsistency/lack of follow through and lack of training for high need trauma students. One could argue findings suggest the aforementioned obstacles contribute heavily to the theme of teacher and principal perceptions that SWPBIS is ineffective and simply does not work to improve student behavior and overall culture and climate at DHES.

An analysis of TELL Survey (2017) teacher perception data collected by the state where DHES is located provides additional perception data that student behavior at DHES is not showing improvement and, is in fact, growing more unsafe. (See Table 2) 
Supports to SPWIS effectiveness were more difficult for teachers and the principal to identify. There was agreement among all study participants that there is a common language and expectations for student behavior that support SWPBIS at Height Elementary School. Unfortunately, although all participants acknowledged the presence of these supports, issues with inconsistency still prove to challenge their successful implementation and sustainability.

Overall, the principal and teachers agreed that working with students from high crime, high poverty urban neighborhoods makes it difficult to create a safe school environment using SWPBIS exclusively. Teachers and the principal alike perceive the need for more specialized training to meet the challenging needs of students who are victims of trauma and have undiagnosed mental health needs. While some aspects of SWPBIS have been implemented successfully, there is perception data indicating inconsistency from teachers and administration at DHEs continues to plague sustainability and overall effectiveness. Essentially, teachers and the principal in this study perceive SWPBIS alone is not effective in urban, low achieving elementary schools who serve students from traumatic backgrounds because they do not have the ability to control impulsive emotions that lead to unsafe school environments.

\section{Summary}

This chapter provided an in-depth look at the experiences and perceptions of 11 participants who engaged in SWPBIS at Dorothy Height Elementary School. The purpose of this single site case study was to explore and gather teacher and principal perceptions of how a SWPBIS framework impacts overall school culture and climate in an urban, persistently low achieving elementary school. The participants included teachers, 
inclusive of coaches and interventionists, and the principal. This researcher intended to interview the assistant principal at DHES, but the position was vacant at the time of this study. Interviews were face-to-face and relevant documents were analyzed to identify teacher and principal perceptions and behavioral data that influence the school climate.

A thorough analysis of the data uncovered four essential themes: (a) SWPBIS is ineffective/does not work, (b) high levels of teacher frustration, (c) increase in violent student behaviors, and (d) no consequences/students rewarded for inappropriate behaviors. Obstacles and supports to the implementation, sustainability and overall effectiveness of SWPBIS emerged through face-to-face interviews, analysis of Office Discipline Referrals or ODRs known as Behavior Intervention Logs or BILs at DHES, and TELL Survey data from 2017. Through a three-cycle coding process and data analysis, the following obstacles to SWPBIS effectiveness were identified: (a) urban/high poverty students, (b) inconsistency/lack of follow through, and (c) lack of training for high need trauma students. Supports to the effectiveness of SWPBIS include: (a) common language, and (b) expectations.

This case study voiced that teachers and the principal at DHES perceive SWPBIS is ineffective and it does not have a positive impact on school climate. Participants provided candid, honest experiences in their interviews describing the ineffectiveness of SWPBIS to address the needs of students in urban, high poverty schools and neighborhoods. The study revealed extremely high levels of teacher frustration with out of control and often times violent student behaviors that make the school an unsafe environment. Teachers and the principal acknowledged an increase in more violent student behaviors is negatively impacting the learning environment and making the 
school unsafe for students, teachers, staff and administrators. Finally, the fourth major theme uncovered that teachers perceive a lack of consequences for inappropriate behaviors and that the administrators reward students for inappropriate behaviors. In addition, this study acknowledged common language and expectations exist schoolwide through the use of the PAWS acronym support the effectiveness of SWPBIS and that students can articulate the behavioral expectations for the classroom, hallway, cafeteria and playground. Unfortunately, the principal acknowledged her concerns about whether or not students have internalized the expectations and are putting them into practice. Overall, this study revealed participants perceived several obstacles preventing SWPBIS implementation, sustainability and overall effectiveness. Teachers reported that additional challenges of working with urban, high poverty students was problematic because these students bring additional home life issues to the school environment that often cause them to exhibit disruptive and inappropriate behaviors. Data triangulation, through the use of TELL Survey data validates teacher perceptions that classroom disruptions and challenging student behavior continue to prevent teachers from providing high quality academic instruction despite the use of SWPBIS framework at DHES. 


\section{CHAPTER V \\ DISCUSSION \& IMPLICATIONS \\ Overview}

Discipline problems in American schools impede learning for all students and lead to increased teacher frustrations and unsafe learning environments. Skiba et al. (2014) found teachers in high poverty, urban schools likely spend more time addressing student discipline issues including disruptive behavior, fighting, drug use and/or sales and weapons possession. Despite the increase in student behavior problems, schools have moved away from the zero-tolerance policies of the 1990s to the more proactive SWPBIS framework to support student behavior and improve school culture and climate. SWPBIS employs a building-wide approach to improve academic and social outcomes for students through the use of multi-tiered systems of support (Horner \& Sugai 2015).

The purpose of this collective case study was to ascertain deeper understanding of teacher and administrator perceptions of the effectiveness of a SWPBIS framework in an urban, persistently low-achieving elementary school. Distinctively, this study explored what teachers and administrators perceive as obstacles and supports to SWPBIS implementation, sustainability and effectiveness as measured by its positive impact on culture and climate. This chapter provided a discussion of the findings as related to the research literature and theoretical framework. The theoretical and empirical implications will then be discussed. Lastly, the limitations of this collective case study and recommendations for future research will be proposed. 


\section{Discussion}

The following is a discussion of the findings in relation to the theoretical and empirical review in Chapter two. The literature review contained a thorough examination of the basic tenets of SWPBIS and relevant research on teacher and administrator perceptions of SWPBIS and how it supports a host environment resulting in a positive culture and climate and student academic success. The collective case study utilized Fuller's Concern-Based-Adoption-Model (CBAM) to examine how teacher and principal perceptions of SWPBIS impact its effectiveness. Study participants responded to interview questions designed to uncover concerns about the effectiveness of SWPBIS in their urban, persistently low achieving elementary school and identify obstacles and supports to SWPBIS sustainability, implementation and overall impact on culture and climate.

The theoretical framework selected for this study, pragmatism, examined if teachers and the principal at a high poverty, urban elementary school perceived SWPBIS works to improve negative student behaviors and the overall school climate.

Additionally, the literature review investigated past and current issues with school discipline, SWPBIS framework origins and foundational precepts and the additional impact that urban school context contributes to SWPBIS effectiveness. The findings from this study inform the research questions based upon the theoretic framework.

\section{Discussion of the Theoretical Framework}

Pragmatic researchers ask questions concerned with the "what" and the "how" of research and the anticipated consequences the research may bring to light (Cherryholmes, 1992). The SWPBIS framework promotes the use of proactive behavior strategies to 
promote positive student behaviors and healthy school climates. In this case study, anticipated consequences include teacher and principal perceptions of whether or not SWPBIS works to increase positive student behavior, which in turn leads to an overall positive school culture and climate and ultimately, increased student academic success. The findings from the present case study at an urban, persistently low performing elementary school indicated that the implementation of SWPBIS did not decrease the frequency of negative student behaviors and, since its implementation, student behaviors have become more disruptive and violent. From a pragmatic perspective, findings showed participants feel SWPBIS is ineffective and simply does not "work" to decrease disruptive and violent student behaviors and create a welcoming and safe school learning environment.

Theoretically, pragmatic inquiry focuses on assessing the efficacy or practicality of the research topic to determine if it achieves its intended goal. In this case, the practicality of continuing to implement SWPBIS as the primary strategy to improve school safety, culture and climate by decreasing negative student behaviors. Findings indicated the teachers and principal at the urban elementary school selected for this case study do not feel SWPBIS is effective and other school-wide behavioral options should be explored to address the high needs student population. Contextually, urban schools have increased rates of poverty, fewer monetary resources, high rates of teacher turnover and greater achievement gaps in reading and math as compared to suburban and rural schools (Council of the Great City Schools, 2013).

From a pragmatic standpoint, this study's findings show a majority of teachers and the principal perceive the contextual factors of the school's location in a high 
poverty, high crime urban neighborhood creates a substantial obstacle to the effectiveness of SWPBIS to create a positive school climate. Contextual background information obtained during semi-structured interviews provided information about participants' years of teaching experience and experience working in urban, suburban and rural schools. Nine of the 10 teacher participants and the principal at DHES possess nine or more years of experience working in multiple contexts, in addition to urban schools, and study findings indicate shared beliefs that students who live in high poverty, high crime, and urban communities pose additional challenges to the implementation, sustainability and effectiveness of SWPBIS.

A gap exists in research on SWPBIS effectiveness in large, urban public schools with increased ethnic and cultural diversity, poverty and antisocial behavior is not as prevalent as research on SWPBIS effectiveness in suburban and rural schools. Participant perceptions in this study align with this call for additional research as teachers and the principal both assert that urban schools struggle with student behavior and discipline due to traumatic home lives. In addition, a majority of DHES teachers perceive urban high poverty students are less responsive to positive praise, feedback and rewards for following SWPBIS expectations.

The theoretical framework selected for the present study, pragmatism, contributes to the existing research on the effectiveness of SWPBIS because it provides teacher and principal perceptions about SWPBIS in an elementary school located in a large, urban public school district. Study findings confirm the existing research that urban schools require more training and groundwork prior to and during SWPBIS implementation and these same schools face additional obstacles to successfully implement SWPBIS. 


\section{Discussion of the Related Literature}

An orderly, safe climate conductive to learning is one of five essential elements or correlates of an effective school (Edmonds, 1982). In the nineties, public schools in the United States experienced sharp increases in student misbehavior and violence, which resulted in the adoption of zero-tolerance policies in an attempt to ensure school safety. These tougher policies designed to hold students accountable for disruptive, violent and, at times, illegal behaviors, lead to what is commonly known at the "school to prison pipeline", backfired as critics decried the disproportionate suspension and arrest rates for African American males for largely unjustified reasons.

Skiba and Peterson (2000) found that zero tolerance policies were imbued with ambiguous descriptions of the difference between minor offenses and severe offense. In an effort to right this wrong, education policy makers nationwide adopted School-Wide Positive Behavior Interventions and Supports (SWPBIS) in the early years of the $21^{\text {st }}$ century. SWPBIS promotes a systematic approach to anticipate problem behaviors, eliminate triggers and teach social skills strategies and behaviors resulting in positive, sustainable changes in student behavior. Unfortunately, since the nationwide

implementation of SWPBIS, the research literature suggests student disruptive behaviors have continued to increase and student discipline and classroom management challenges plague urban schools (McKinney, Campbell-Whatley, \& Kea, 2005).

This continued increase in problem behaviors despite SWPBIS adoption in the majority of public schools nationwide, coupled with the call for more research on SWPBIS in urban schools, prompted the decision to design the present study. This researcher pondered if education policy makers caused the pendulum to swing too far to 
the left or right in efforts to distance behavior policies from zero tolerance initiatives and erred on the side of caution by not holding students accountable for behaviors that disrupt instruction and make schools unsafe. To answer this question, this study sought to bring to light the discerning opinions of those individuals on the frontlines of public education, teachers and principals, to determine if they believe SWPBIS works in urban schools to decrease student misbehavior and violence.

According to the teacher participants and the principal in this study, SWPBIS is ineffective and simply does not work to decrease problem student behaviors that continue to grow more violent each year. A common theme among teacher participants and the principal is students from high, poverty urban, high crime neighborhoods often bring additional stress and trauma from dysfunctional home lives which prevent the proactive SWPBIS framework from achieving success. Specifically, the principal shared her perception that outside factors and mental health issues contribute to the ineffectiveness of SWPBIS.

Skiba et al. (2014) found teachers in high poverty, urban schools spend more time dealing with student discipline problems such as disruptive behavior, fighting and use of possession of weapons or drugs. Teacher participants also reported high levels of teacher frustration due to constant interruptions during teaching, students being rewarded for inappropriate behaviors, increasingly violent behaviors and a lack of consistent consequences from administration.

Despite conflicting research, Office Discipline Referrals (ODRs) are the most common measure used to monitor and analyze problem behavior in SWPBIS (Bohanon et al., 2006). School data from Behavior Intervention Logs (BILs) or office referrals, 
illustrates an increase in suspensions over the past five years despite a decrease in the number of office discipline referrals (ODRs). Thus, suspensions for more violent behaviors, such as fights, continue to increase while the number of ODRs decreases as compared from the previous year. Teachers expressed frustration in response to administration directives to handle more behavior problems in the classroom without calling for behavior support assistance. Thus, the decrease in ODRs does not necessarily demonstrate an increase in positive school climate, but a concerted effort from administrators to reduce the number of office referrals completed and thus reported to central office and, therefore, available to the public via open records request.

Mathison (1988) found good researchers are obligated to triangulate, or use multiple methods, data sources, to enhance the validity of research findings. In an effort to triangulate teacher perception data for this study, this researcher compared TELL Survey data from 2015 and 2017. Findings indicated only 3.2\% and 3.0 \% of teachers felt students followed school rules. In addition, 2017 TELL Survey data showed only 15.2 \% of teachers believed school administrators consistently enforced rules of student conduct. This data supports themes of teacher frustration and perceptions that SWPBIS was ineffective at Dorothy Height Elementary School because student behaviors have continually increased and become more violent.

Study participants identified high percentages of students with trauma and mental health needs as an obstacle to SWPBIS effectiveness. Consistent with these findings, the study revealed teachers and the principal perceive an obstacle to SWPBIS implementation, sustainability and effectiveness is the number of students with mental health issues and high need trauma students who make the school environment unsafe. 
A majority of teachers and the principal conveyed serious concerns that SWPBIS does not provide teachers with the additional training needed to address urban students with increased levels of adverse childhood experiences, i.e., trauma, and this contributes to its ineffectiveness. The principal expressed critical concerns and frustration with SWPBIS and has intervened to provide additional training to teachers in social emotional learning to address students' lack of social skills to control emotions. Additionally, the principal has provided the staff with district and school-based professional development and training in trauma-informed approaches to classroom management. The present study found the teacher participants and the principal perceive SWPBIS to be ineffective. Despite the continued classification of being a SWPBIS school, study participants feel the SWPBIS framework implementation has been inconsistent and lacks trauma training and extremely arduous due to the additional challenges of teaching students from high crime, urban, high poverty backgrounds.

\section{Implications}

The following sections discuss theoretical, empirical and practical implications for this study. These findings may prove advantageous to large, urban public school districts and elmentary, middle and high schools located in high poverty settings when considering the adoption and implementation of SWPBIS . Through the exploration of teacher and administrator perceptions, the results from the present study seek to add to the existing literature regarding SWPBIS implementation, sustaininability and effectiveness in urban, high poverty schools to assist with obstacles and supports that may help and hinder the improvement of school culture and climate. 


\section{Theoretical Implications}

The results of this collective case study provide a response to the pragmatic question of whether or not SWPBIS works to improve student behaviors and overall culture and climate. The present case study found teachers and principals have concerns about the effectiveness of SWPBIS in urban schools and perceive it does not achieve its intended goals. Participants voiced several obstacles to SWPBIS implementation, sustainability and effectiveness including lack of consistency, additional challenges resulting from high poverty, urban students and SWPBIS does not provide specified training needed to provide interventions to student victims of trauma.

From a practical standpoint, SWPBIS effectivess depends on the consistent and proactive application of school wide expectations, positive praise and reinforcement and rewards for adhering to school expectations. Unfortunately, teachers and principals in the present student study feel SWPBIS is ineffective in the context of urban schools because students' come from more dysfunctional homes and communities and are less responsive to positve praise and non-tangible or edible rewards.

\section{Empirical Implications}

This case study expanded research on SWPBIS and measures of its effectiveness on school climate by querying the perceptions of teachers and administrators. This study expands and fills a gap in the existing literature which finds additional training and preparation is required to successfully implement SWPBIS in urban schools. Warren et al. (2003) concluded the unique challenges of urban schools require additional preparation and training before successful PBIS implementation can occur and cited the need for additional research on SWPBIS in urban environments. Using the Concerns- 
Based Adoption Model (CBAM), which is a framework for understanding the evolution of educators' questions and concerns during the implementation of research-based practices (Fuller, 1969; Hall \& Hord, 2011; Roach et al., 2009), teacher participants in the present study shared numerous concerns about the lack of specific and targeted training in the SWPBIS framework to effectively respond to students in urban schools.

In addition, this study reinforced a previous study which found children living in high poverty areas and attending urban schools are at risk of exhibiting antisocial behavior as a result of exposure to significant family and community-based risk factors such as: exposure to physical and sexual abuse, family exposure to drugs and alcohol, and negative encounters with police (McCurdy, Mannella \& Eldridge, 2003). Participants in the present study perceived students from traumatic backgrounds tend to exhibit more antisocial, disruptive and violent behaviors that students who do not reside in high poverty, high crime urban neighborhoods.

\section{Practical Implications}

Findings from the present study identify teacher and principal concerns that arise during the implementation phase of SWPBIS and participant perceptions of factors that support and hinder SWPBIS effectiveness in persistently low performing schools located in urban, high poverty neighborhoods. Specific recommendations for central office personnel, school administrators and teachers are provided below.

\section{Recommendations for Central Office Personnel}

It is important to note that turnover of central office staff and teacher and principal attrition play an intregal part in the effective implementation of any districtwide initiative. Despite these difficulties, central office personnel have a moral 
imperative to exercise care and prudence in making important, and potentially life changing decisions that impact students, teachers, administrators, parents, local businesses and the community at large. As a result of the far reaching consequences of decisions at the district office level, central office administrators, such as the superintendent, assistant superintendents, etc., have a duty to exhaust all resources available to identify existing research based interventions to improve academic, social and behavioral outcomes for students. SWPBIS has been the frontrunner as a framework to improve student behavior and culture and climate since the early $21^{\text {st }}$ century. Unfortuntately, data suggests public schools continue to struggle with increases in disruptions, fighting, and other violent behaviors that make schools unsafe.

This researcher recommends central office personnel perform their due diligence in researching and providing training and support for alternatives to SWPBIS, such as Restorative Practices, Social Emotional Learning and Trauma-Informed Care, that may have studies indicating successful shifts in urban schools to minimize inappropriate behaviors and create safe, positive school environments where all students can reach their full academic potential and graduate prepared for success in life.

\section{Recommendations of School Administrators}

The term school administrators includes principals, assistant principals, counselors and other support personnel. These individuals are charged with ensuring students are safe at school and reach their full academic and social potential to be prepared for college and/or career. In the wake of two decades of increased gun violence in schools, it is a priority that school administrators ensure the safety of all individuals on the campus and it seems common sense to ensure consistency in the implementation of 
the program or framework selected to provide an overall sense of school procedures and protocols with regard to safety. It is this researcher's recommendation that principals at urban schools regard SWPBIS as a priority and ensure all staff members receive in-depth initial training prior to the implementation of SWPBIS and on-going training and professional development to ensure staff buy-in and consistency with language and expectations. The reponsibility for SWPBIS effectiveness ultimately rests with the school principal, as the building administrator, to achieve stake holder buy-in, hold staff members accountable to support school-wode initiatives, progress monitor implementation and sustainability and initiate the appropriate actions necessary to achieve the desired result.

This researcher recommends principals take an active role in data team meetings to discuss suspension and ODR data to make mid-course corrections, such as shifting additional personnel to monitor "hot spots" in the building, to respond to the changing needs of the students resulting from high levels transiency, poverty, urban crime, and high needs trauma.

\section{Recommendations for Teachers}

Teachers aspiring to work in large, urban school districts could benefit from the findings of this collective case study through analysis of the other teachers' perceptions of obstacles and supports that impact SWPBIS effectiveness. Having the knowledge that additional challenges exist in urban schools provides teachers with the opportunity to seek out and take advantage of district and school-based training on SWPBIS, socialemotional learning, trauma-informed schools and cultural competency strategies to better meet the needs of students from urban, high trauma backgrounds. The more prepared 
teachers are to understand the backgrounds and challenges their students makes them more sensistive to students' home lives and understand the contributing factors to their negative behaviors.

\section{Study Modifications and Limitations}

This collective case study includes weaknesses and limitations that fundamentally exist in qualitative research studies. First, the majority of the data from the present study is derived from participant face-to-face interviews and, thus, the data consists of their feelings, perceptions and overall concerns regarding SWPBIS effectiveness. Since perception is reality, participant beliefs exist within their schema of life experiences which may or may not be tainted by negative past experiences with SWPBIS, certain groups of people and various school contexts such as: urban, rural or suburban. In addition to possible teacher bias, this researcher creates a limitation on the presentation of the study results because extra care must be taken to ensure biases and pre-conceived notions are not unconsciously injected into the findings and interpretation of the data.

A second limitation on the present study involves transferability of the findings to apply to SWPBIS effectiveness in other urban, high poverty elementary schools. In this collective case study, teachers and the principal at a single case study site shared concerns regarding SWPBIS implementation, sustainability and effectiveness. This scholar took care to attempt to provide enough detail about the study design, methodology and data analysis techniques that another researcher could replicate this same study to determine if the outcomes are similar or different. Unfortuntately, contextual factors, district supports, differences in the leadership style, and capacity of the building leader, teacher biases and other demographic factors of the student 
population create difficulty to generalize information gained from the findings of this study. Researcher positionality creates a specific limitation for the present study because the researcher works in the school where the case study took place as a school turnaround specialist and has developed her own opinions based upon two years of observations and experiences in the building. Despite this obstacle, this researcher feels that she maintained objectivity in the presentation of the findings through the use of data from existing research literature, participant interviews and school document analysis for triangulation to reach the conclusions presented here. If any bias or researcher positionality dangers exist, this researcher contends unitentional inclusion through seen, unseen and/or unforseen admittance.

\section{Recommendations for Future Research}

This case study provided purposeful insight into the perceptions of teachers and administrators in the implementation and sustainability stages of SWPBIS. This research added to the existing body of literature regarding SWPBS effectiveness in large, urban public schools, obstacles and supports to the process and recommendations for future research. This scholar recommends additional studies be conducted in urban elementary schools similar to the urban elementary school depicted in this study to ascertain similarities and differences in participant perceptions of SWPBIS effectiveness, sustainability, implementation and impact on improving school culture and climate.

Additional studies in similar contexts may provide opportunities to narrow down issues with the SWPBIS framework's ability to positively impact students in urban, lowperforming schools located in high crime areas and eliminate issues of variance in administrator effectivess, district support and training in implementation and teacher 
effectivess. These additional perspectives would help the address the pragmatic question of whether or not the SWPBIS framework provides all components necessary to create positive host environments for strong culture and climate or if additional programs or initiatives must be utilized to supplement SWPBIS to meet the needs to all students and achieve the desired result.

\section{Summary and Conclusion}

The purpose of this collective case study was to examine teacher and principal perceptions of whether or not SWPBIS works to reduce negative student behaviors and create a safe and positive school climate in urban, low performing schools. Qualitative case study design and methodology was appropriate for this study because they allow researchers to explore a problem or an issue, to identify variables not easily determined or to hear silenced voices (Creswell, 2013, p.47-48). This study sought to ascertain the quiet voices of teachers and the principal working in an urban school plagued by disruptive behavior as a result of the additional challenges of poverty, trauma and living in high crime neighborhoods.

Semi-structured interviews yielded rich data descriptions of student behaviors, teacher and principal concerns, frustrations, experiences, and day to day observations that shape participant perceptions of the effectiveness of SWPBIS in the challenging environment of urban, high poverty schools. The perceptions of 11 participants were explored and, as a result of the thematic analysis process, four major themes emerged: SWPBIS is ineffective, increasingly high levels of teacher frustration, more violent student behaviors and lack of consequences/students rewarded for inappropriate behaviors. 
Although prior research studies regarding SWPBIS implementation, sustainability and effectiveness have been conducted, the majority have been quantitative in design and few have focused on teacher and principal concerns in urban settings. This study achieved its goal in providing a platform for the often ignored voices of teachers and the principal tasked with the increased challenge of implementing the SWPBIS framework in their urban school. SWPBIS is a research-based framework that has achieved positive results in the past twenty years to reduce student misbehaviors and improve school climate. But, the voices of the participants in this case study shed light on possible implications of SWPBIS' lack of positive results in the context of urban schools filled with students plagued by generational poverty, violent neighborhood crime, drug sales and use and the resulting high needs trauma.

Previous research studies on SWPBIS focused on how training, implementation and sustainability impact overall effectiveness to create a safe, welcoming culture and climate and positive relationships with the parents and community. This study also acknowledged these important factors, but, through the voices of teachers and the principal, attempted to ascertain whether or not SWPBIS is enough to combat the unique challenges teachers and principals working in urban schools face on a daily basis. Hopefully, the narratives of these educators who seek to improve educational outcomes for all students whether in urban, rural or surburban contexts, results in additional research on either supplementary programs for SWPBIS to meet the needs of urban sudents or a shift in district and school leadership to "think outside the box" in their approaches to address school-wide discipline and safety in urban schools as a whole. 


\section{REFERENCES}

Adelman H., Taylor L. (2005). Mental health in urban schools. Retrieved from http://smhp.psych.ucla.edu/publications/47\%20Mental $\% 20 \mathrm{Health} \% 20 \mathrm{in} \% 20$ Urban\%20Schools.pdf

Advancement Project \& the Civil Rights Project. (2000). Opportunities suspended: The devastating consequences of zero tolerance and school discipline policies. Boston, MA: Harvard University.

Andreou, T. E., McIntosh, K., Ross, S. W., \& Kahn, J. D. (2015). Critical incidents in sustaining school-wide positive behavioral interventions and supports. The Journal of Special Education, 49(3), 157-167.

Axelrod, S., Moyer, L., \& Berry, B. (1990). Why teachers do not use behavior modification procedures. Journal of Educational and Psychological Consultation, 1(4), 309-320.

Balfanz, R., Byrnes, V., \& Fox, J. H. (2015). Sent home and put off track. Closing the school discipline gap: Equitable remedies for excessive exclusion, 17-30.

Bankston, C., \& Caldas, S. J. (1996). Majority African American schools and social injustice: The influence of de facto segregation on academic achievement. Social Forces, 75(2), 535-555.

Barnes, G., Crowe, E., \& Schaefer, B. (2007). The cost of teacher turnover in five school districts: A pilot study. Washington, DC: National Commission on Teaching and America's Future.

Baxter, P., \& Jack, S. (2008). Qualitative case study methodology: Study design and implementation for novice researchers. The Qualitative Report, 13(4), 544-559. 
Bazemore, G., \& Umbreit, M. (2001). A comparison of four restorative conferencing models. Juvenile Justice Bulletin.

Bohanon, H., Fenning, P., Carney, K. L., Minnis-Kim, M. J., Anderson-Harris, S., Moroz, K. B., Pigott, T. D. (2006). Schoolwide application of positive behavior support in an urban high school: A case study. Journal of Positive Behavior Interventions, 8, 131-145.

Bowditch, C. (1993). Getting rid of troublemakers: High school disciplinary procedures and the production of dropouts. Social Problems, 40(4), 493-509.

Bowles, S., \& Gintis, H. (1976). Schooling in capitalist America (Vol. 57). New York: Basic Books.

Bradshaw, C. P., Mitchell, M. M., \& Leaf, P. J. (2010). Examining the effects of schoolwide positive behavioral interventions and supports on student outcomes results from a randomized controlled effectiveness trial in elementary schools. Journal of Positive Behavior Interventions, 12(3), 133-148.

Brandt, R. C., Chitiyo, M., \& May, M. E. (2014). Measures used in assessing outcomes of school-wide positive behaviour support. Journal of Research in Special Educational Needs, 14(4), 229-238.

Brookmeyer, K. A., Fanti, K. A., \& Henrich, C. C. (2006). Schools, parents, and youth violence: A multilevel, ecological analysis. Journal of Clinical Child and Adolescent Psychology, 35(4), 504-514.

Brookover, W. B., \& Erickson, E. L. (1969). Society, schools, and learning. Allyn and Bacon. 
Byrne, B. M. (1999). The nomological network of teacher burnout: A literature review and empirically validated model. Understanding and Preventing Teacher Burnout, 15-37.

Cameron, L., \& Thorsborne, M. (2001). Restorative justice and school discipline: Mutually exclusive? Restorative Justice and Civil Society, 180-194.

Casella, R. (2001). "Being down": Challenging violence in urban schools. Teachers College Press.

Cashin, S. (2014). Place, not race: A new vision of opportunity in America. Boston: Beacon Press.

Charmaz, K. (2001). Grounded theory: Methodology and theory construction. In the International Encyclopedia of the Social \& Behavioral Sciences. (Vol. 1, pp. 6396-6399). Elsevier Science, Ltd.

Cherryholmes, C. H. (1992). Notes on pragmatism and scientific realism. Educational Researcher, 13-17.

Children's Defense Fund. (1974). Children out of school in America. Washington, DC.

Christie, C. A., Nelson, C. M., \& Jolivette, K. (2004). School characteristics related to the use of suspension. Education and Treatment of Children, 509-526.

Clark, C. (2004). Diversity initiatives in higher education: Multicultural education as a tool for reclaiming schools organized as breeding grounds for prisons. Multicultural Education, 11(3), 50-53.

Cohen, J., McCabe, L., Michelli, N. M., \& Pickeral, T. (2009). School climate: Research, policy, practice, and teacher education. Teachers college record, 111(1), 180-213. 
Collier, C., \& Henriksen, Jr, R. C. (2012). Teachers' perceptions of a multiple high-risk behavior prevention program and delivery of universal programming. The Qualitative Report, 17, 1-19

Combs, A. W. (1982). Affective education or none at all. Educational Leadership, 39(7), 495-97.

Corallo, C., \& McDonald, D. (2001). What works with low-performing schools: A review of research literature on low-performing schools.

Creswell, J. W. (2007). Qualitative inquiry and research design: Choosing among five approaches $\left(2^{\text {nd }}\right.$ ed.). Thousand Oaks, CA: Sage.

Creswell, J. W., Hanson, W. E., Plano, V. L. C., \& Morales, A. (2007). Qualitative research designs selection and implementation. The counseling psychologist, 35(2), 236-264.

Creswell, J. W. (2009). Research design: Qualitative, quantitative, and mixed methods approaches ( $3^{\text {rd }}$ ed.). Thousand Oaks, CA: Sage.

Creswell, J.W., \& Plano-Clark, V. L. (2011). Designing and conducting mixed methods research ( $2^{\text {nd }}$ ed.). Thousand Oaks, CA: Sage.

Creswell, J. W. (2013). Qualitative inquiry and research design: Choosing among five approaches. Sage.

Council of the Great City Schools (2013). Beating the odds. Retrieved from https://www.cgcs.org/cms/lib/DC00001581/Centricity/Domain/4/BTO2014.pdf.

Daniels, J. A., Bradley, M. C., \& Hays, M. (2007). The impact of school violence on school personnel: Implications for psychologists. Professional Psychology: Research and Practice, 38, $652-659$. 
Debnam, K. J., Pas, E. T., \& Bradshaw, C. P. (2013). Factors influencing staff perceptions of administrator support for tier 2 and 3 interventions: A multilevel perspective. Journal of Emotional and Behavioral Disorders, 21(2), 116-126.

Devoe, J. F., Peter, K., Kaufman, P., Miller, A., Noonan, M., Snyder, T. D., \& Baum, K. (2004). Indicators of School Crime and Safety, 2004. NCES 2005-002. National Center for Education Statistics.

Devine, J. (1996). Maximum security: The culture of violence in inner-city schools. University of Chicago Press.

Dewey, J. (1958). Experience and education. New York: Macmillan.

Dishion, T. J., French, D. C., \& Patterson, G. R. (1995). The development and ecology of antisocial behavior. John Wiley \& Sons.

Dishion, T. J., \& Dodge, K. A. (2005). Peer contagion in interventions for children and adolescents: Moving towards an understanding of the ecology and dynamics of change. Journal of abnormal child psychology, 33(3), 395-400.

Dolph, D. (2017). Challenges and opportunities for school improvement:

Recommendations for urban school principals. Education and Urban Society, 49(4), 363-387.

Duke, D. L. (1990). School organization, leadership, and student behavior. Student discipline strategies: Research and practice, 19-46.

Edmonds, R. (1982). Programs of school improvement: An overview. Educational Leadership, 40, 4-11.

Edwards, D. (1995). The school counselor's role in helping teachers and students belong. Elementary School Guidance \& Counseling, 29(3), 191-197. 
Emihovich, C. A. (1983). The color of misbehaving: Two case studies of deviant boys. Journal of Black Studies, 13(3), 259-274.

Fenning, P., \& Rose, J. (2007). Overrepresentation of african american students in exclusionary discipline the role of school policy. Urban Education, 42(6), 536559.

Ferguson, A. A. (2001). Bad boys: Public schools in the making of black masculinity. University of Michigan Press.

Feuerborn, L. L., Wallace, C., \& Tyre, A. D. (2016). A qualitative analysis of middle and high school teacher perceptions of schoolwide positive behavior supports. Journal of Positive Behavior Interventions, 18(4), 219-229.

Fine, M., Burns, A., Payne, Y., \& Torre, M. (2004). Civics lessons: The color and class of betrayal. Working method: Research and social justice, 53 .

Finn, J. D. (1989). Withdrawing from school. Review of educational research, 59(2), 117-142.

Foucault, M. (1977). Discipline and punish: The birth of the prison. Vintage.

Fox, L. E. (2015). Parental wealth and the black-white mobility gap in the US. Review of Income and Wealth, 62(4), 706-723.

Fuller, F. F. (1969). Concerns of teachers: A developmental conceptualization. American educational research journal, 207-226.

Gabbard, D. (2000). Knowledge and power in the global economy: Politics and the rhetoric of school reform. Lawrence Erlbaum Associates.

Gall, M. D., Borg, W. R., \& Gall, J. P. (1996). Educational research: An introduction. Longman Publishing. 
Gall, G., \& Gall, J. P. Borg. (2003). Educational research: An introduction.

Gaustad, J. (1992). "School Discipline.” Striving for excellence: The national education goals. Vol. II.

González, J. M. \& Szecsy, E. M. (2004). The condition of minority access and participation in Arizona: 2004.

Goodenow, C., \& Grady, K. E. (1993). The relationship of school belonging and friends' values to academic motivation among urban adolescent students. The Journal of Experimental Education, 62(1), 60-71.

Gottfredson, D. C. (2001). Schools and delinquency. Cambridge University Press.

Gray, L., \& Taie, S. (2015). Public school teacher attrition and mobility in the first five years: Results from the first through fifth waves of the 2007-08 beginning teacher longitudinal study. National Center for Education Statistics. Retrieved from http://files.eric.ed.gov/fulltext/ED556348.pdf.

Gregory, J. F. (1997). Three strikes and they're out: African American boys and American schools' responses to misbehavior. International Journal of Adolescence and Youth, 7(1), 25-34.

Guerra, N. G., Huesmann, L. R., Tolan, P. H., Van Acker, R., \& Eron, L. D. (1995). Stressful events and individual beliefs as correlates of economic disadvantage and aggression among urban children. Journal of Consulting and Clinical Psychology, 63(4), 518.

Hacker, A. (2010). Two nations: Black and white, separate, hostile, unequal. Simon and Schuster. 
Hall, G. E., \& Hord, S. M. (2011). Implementing change: Patterns, principles, and potholes ( $3^{\text {rd }}$ ed.). Boston, MA: Allyn \& Bacon.

Handler, M. W., Rey, J., Connell, J., Thier, K., Feinberg, A., \& Putnam, R. (2007). Practical considerations in creating school-wide positive behavior support in public schools. Psychology in the Schools, 44(1), 29-39.

Hanna, J. L. (1988). Disruptive school behavior: Class, race, and culture. New York: Holmes \& Meier.

Harris, E. (2016, April 7). New York education dept. is sued over violence in schools. The New York Times. Retrieved from: http://www.nytimes.com/2016/04/08/nyregion/new-york-education-dept-is-suedover-violence-in-schools.html?_r=0.

Hirschi, T. (1969). Causes of delinquency. Berkeley, CA: University of California Press.

Horner, R. H., \& Sugai, G. (2015). School-wide PBIS: An example of applied behavior analysis implemented at a scale of social importance. Behavior Analysis in Practice, $8(1), 80-85$.

Irvin, L. K., Tobin, T. J., Sprague, J. R., Sugai, G., \& Vincent, C. G. (2004). Validity of office discipline referral measures as indices of school-wide behavioral status and effects of school-wide behavioral interventions. Journal of Positive Behavior Interventions, 6(3), 131-147.

Irvine, J. J. (1990). Black students and school failure. Policies, practices, and prescriptions. Greenwood Press, Inc.

Johnson, J. A. (1995). Life after death: Critical pedagogy in an urban classroom. Harvard Educational Review, 65(2), 213-231. 
Johnson, T., Boyden, J. E., \& Pittz, W. J. (2001). Racial Profiling and Punishment in US Public Schools: How Zero Tolerance Policies and High Stakes Testing Subvert Academic Excellence and Racial Equity Research Report [and] Executive Summary.

Johnson, S. M., Marietta, G., Higgins, M. C., Mapp, K. L., \& Grossman, A. (2015). Achieving coherence in district improvement: Managing the relationship between the central office and schools. Harvard Education Press. Cambridge, MA.

Johnston, R. (2000). Federal data highlight disparities in discipline. Education Week, 19(41), 3 .

Kam, C., Greenberg, M. T., \& Walls, C. T. (2003). Examining the role of implementation quality in school-based prevention using the paths curriculum. Prevention Science, 4, 55-63.

Kentucky Center for Instructional Discipline. (2015). About us. Retrieved from http:// www.kypbis.org/about-us

Kentucky Center for School Safety (2016). Kentucky 2014-2015 thirteenth annual safe schools data project. Retrieved from https://kycss.org/pdfsdocs/FY15\%20Safe\%20Schools\%20Data\%20Report\%20Finalrevised\%2010\%2015am\%208-18-2016.pdf

Kentucky Department of Education Division of Student Success. (2014). 2013-14 safe schools annual statistical report. Retrieved from education.ky.gov/school/sdfs/Documents/2013-14 
Kentucky Department of Education Division of Student Success. (2015). 2014-15 safe schools annual statistical report. Retrieved from http://education.ky.gov/school/sdfs/Documents/2014-2015

Kincaid, D., Childs, K., Blase, K., \& Wallace, F. (2007). Identifying barriers and facilitators in implementing schoolwide positive behavior support. Journal of Positive Behavior Interventions, 9(3), 174-184.

Kincheloe, J. (2010). Chapter One: Why a book on urban education? In Counterpoints, (Vol. 215). 19 urban questions: Teaching in the city (1-25). Peter Lang AG.

Lassen, S. R., Steele, M. M., \& Sailor, W. (2006). The relationship of school-wide positive behavior support to academic achievement in an urban middle school. Psychology in the Schools, 43(6), 701-712.

Leone, P., Mayer, M., Malmgren, K., \& Meisel, S. (2000). School violence and disruption: Rhetoric, reality, and reasonable balance. Focus on Exceptional Children, 33(1), 1.

Lippman, L., McArthur, E., \& Burns, S. (1996). Urban schools: The challenge of location and poverty. Diane Publishing.

Loewen, J. (2008). Does My Town Have a Racist Past? Teaching Tolerance, 33, 22-27.

Lohrmann, S., Forman, S., Martin, S., \& Palmieri, M. (2008). Understanding school personnel's resistance to adopting schoolwide positive behavior support at a universal level of intervention. Journal of Positive Behavior Interventions, 10(4), 256-269. 
Luiselli, J. K., Putnam, R. F., Handler, M. W., \& Feinberg, A. B. (2005). Whole-school positive behaviour support: effects on student discipline problems and academic performance. Educational Psychology, 25(2-3), 183-198.

Markow, D., \& Cooper, M. (2008). The Metlife survey of the American teacher past, present and future. New York, NY: Metlife Insurance Company.

Maslow, A. (1962). Toward a psychology of belonging. Princeton, MJ: Van Nostrand.

Mathison, S. (1988). Why triangulate? Educational researcher, 17(2), 13-17.

Mayer, G. R. (1995). Preventing antisocial behavior in the schools. Journal of applied behavior analysis, 28(4), 467-478.

McCadden, B. M. (1998). Why is Michael always getting timed out? Race, class, and the disciplining of other people's children. In RE Butchart \& B. McEwan (Eds.), Classroom discipline in American schools: Problems and possibilities for democratic education, 109-134.

McCaslin, M. L., \& Given, L. M. (2008). Pragmatism. The sage encyclopedia of qualitative research methods, 672-676.

McCurdy, B. L., Mannella, M. C., \& Eldridge, N. (2003). Positive behavior support in urban schools: Can we prevent the escalation of antisocial behavior. Journal of Positive Behavior Interventions, 5(3), 158-170.

McFadden, A. C., Marsh, G. E., Price, B. J., \& Hwang, Y. (1992). A study of race and gender bias in the punishment of school children. Education and Treatment of Children, 140-146.

McIntosh, K., Predy, L. K., Upreti, G., Hume, A. E., Turri, M. G., \& Mathews, S. (2014). Perceptions of contextual features related to implementation and sustainability of 
school-wide positive behavior support. Journal of Positive Behavior Interventions, 16(1), 31-43.

McKinney, S. E., Campbell-Whatley, G. D., \& Kea, C. D. (2005). Managing student behavior in urban classrooms: The role of teacher abc assessments. The Clearing House, 79, 16-20.

McMahon, S. D., Martinez, A., Espelage, D., Rose, C., Reddy, L. A., Lane, K., ... \& Brown, V. (2014). Violence directed against teachers: Results from a national survey. Psychology in the Schools, 51(7), 753-766.

McNamara, C. (2009). General guidelines for conducting interviews.

Merriam, S. B. (1998). Qualitative research and case study applications in education. San Francisco, CA: Jossey-Bass.

Miles, M. B., Huberman, A. M., \& Saldaña, J. (2013). Qualitative data analysis: A methods sourcebook. SAGE Publications, Incorporated.

Milner, H. R. (2006). Preservice teachers' learning about cultural and racial diversity Implications for urban education. Urban Education, 41(4), 343-375.

Monroe, C. R. (2005). Why are" bad boys" always Black? Causes of disproportionality in school discipline and recommendations for change. The Clearing House: A Journal of Educational Strategies, Issues and Ideas, 79 (1), 45-50.

National Center for Education Statistics. Urban Schools: The challenge of location and poverty. (1996) Retrieved from http://nces.ed.gov/pubs/96184all.pdf.

National Center for Education Statistics. Violence and crime at school: Public school reports. (2004) Retrieved from http://files.eric.ed.gov/fulltext/ED483086.pdf. 
National Center for Education Statistics. (2009). Crime, violence, discipline and safety in U.S. public schools. Retrieved from ttp://files.eric.ed.gov/fulltext/ED505156.pdf. National Center for Education Statistics. (2014) Indicators of School Crime and Safety: 2013. Retrieved from http://nces.ed.gov/.

National Center for Education Statistics. Indicators of School Crime and Safety: 2015. (2016) Retrieved from https://nces.ed.gov/pubsearch/pubsinfo.asp?pubid=2016079.

Nichols, E. J. (2013). Cultural Competence in America's Schools: Leadership, Engagement and Understanding. Information Age Pub.

Noguera, P. (1995). Preventing and producing violence: A critical analysis of responses to school violence. Harvard Educational Review, 65(2), 189-213.

Noguera, P. A. (2003). Schools, prisons, and social implications of punishment: Rethinking disciplinary practices. Theory into Practice, 42(4), 341-350.

Oakes, J. (1985). Keeping track. Yale University Press.

Orfield, M. (2002). American metropolitics: The new suburban reality. Washington, DC: Brookings Institution Press.

Osher, D., Bear, G. G., Sprague, J. R., \& Doyle, W. (2010). How can we improve school discipline? Educational Researcher, 39(1), 48-58.

Osher, D., Quinn, M. M., Poirer, J. R., \& Rutherford, R. (2003). Deconstructing the pipeline: Using efficacy and effectiveness data and cost-benefit analyses to reduce minority youth incarceration. New Directions in Youth Development, 99, 91-120. 
Pas, E. T., \& Bradshaw, C. P. (2012). Examining the association between implementation and outcomes. The Journal of Behavioral Health Services \& Research, 39(4), 417-433.

Patton, M. Q. (1990). Qualitative Evaluation and Research Methods. SAGE Publications, Inc.

Patton, M.Q. (2002). Qualitative Evaluation \& Research Methods (3 ${ }^{\text {rd }}$ ed.). Thousand Oaks, CA: Sage Publications.

Payne, A. A., Gottfredson, D. C., \& Gottfredson, G. D. (2006). School predictors of the intensity of implementation of school-based prevention programs: Results from a national study. Prevention Science, 7(2), 225-237.

Perry, A. C. (1914). The management of a city school. New York: The Macmillan Company.

Phillips, M. (1932). Some problems of adjustment in the early years of a teacher's life. British Journal of Educational Psychology, 2(3), 237-256.

Price, H. E. (2011). Principal-teacher interactions: How affective relationships shape principal and teacher attitudes. Educational Administration Quarterly, 48, 39-85.

Public Agenda Foundation. (2004). Teaching interrupted: Do discipline policies in today's public schools foster the common good? Retrieved from: http://www.publicagenda.org

Raffaele Mendez, L. (2003). Predictors of suspension and negative school outcomes: A longitudinal investigation. New directions for youth development, 2003(99), 1733. 
Richter, M. M., Lewis, T. J., \& Hagar, J. (2012). The relationship between principal leadership skills and school-wide positive behavior support: An exploratory study. Journal of Positive Behavior Interventions, 14(2), 69-77.

Riffel, L. A. (2011). Positive behavior support at the tertiary level. Thousand Oaks, CA: Corwin.

Riley, J. L., McKevitt, B. C., Shriver, M. D., \& Allen, K. D. (2011). Increasing on-task behavior using teacher attention delivered on a fixed-time schedule. Journal of Behavioral Education, 20(3), 149.

Roach, A., Kratochwill, T., \& Frank, J. (2009). School-based consultants as change facilitators: Adaptation of the concerns-based adoption model (CBAM) to support the implementation of research-based practices. Journal of Educational and Psychological Consultation, 19, 300-320.

Ross, S. W., Romer, N., \& Horner, R. H. (2011). Teacher well-being and the implementation of schoolwide positive behavior interventions and supports. Journal of Positive Behavior Interventions, 1098300711413820.

Rutter, M. (1981). The city and the child. American Journal of Orthopsychiatry, 51(4), 610.

Schwartz, R., \& Rieser, L. (2001). Zero tolerance as mandatory sentencing. Zero tolerance: Resisting the drive for punishment in our schools, 126, 136.

Shaw, S. R., \& Braden, J. P. (1990). Race and gender bias in the administration of corporal punishment. School Psychology Review, 19(3), 378-383.

Simon, J. (2007). Governing through crime: How the war on crime transformed American democracy and created a culture of fear. Oxford University Press. 
Simonsen, B., Sugai, G., \& Negron, M. (2008). Schoolwide positive behavior supports. Teaching Exceptional Children, 40(6), 32-40.

Skiba, R. J., Michael, R. S., Nardo, A. C., \& Peterson, R. (2000). The color of discipline. Policy Research Report, Indiana Education Policy Center.

Skiba, R. J., \& Peterson, R. L. (2000). School discipline at a crossroads: From zero tolerance to early response. Exceptional Children, 66(3), 335-346.

Skiba, R. J., Simmons, A., Staudinger, L., Rausch, M., Dow, G., \& Feggins, R. (2003). Consistent removal: Contributions of school discipline to the school-prison pipeline. School to Prison Pipeline Conference. Boston, MA.

Skiba, R. J., \& Rausch, M. K. (2006). Zero tolerance, suspension, and expulsion: Questions of equity and effectiveness. Handbook of Classroom Management: Research, Practice, and Contemporary Issues, 1063-1089.

Skiba, R. J., Chung, C. G., Trachok, M., Baker, T. L., Sheya, A., \& Hughes, R. L. (2014). Parsing disciplinary disproportionality: Contributions of infraction, student, and school characteristics to out-of-school suspension and expulsion. American Educational Research Journal, 51(4), 640-670.

Smeeding, T. M. (2001). The gap between rich and poor: A cross-national perspective for why inequality matters and what policy can do to alleviate it. National Institute of Population and Social Security Research, 21.

Stake, R. E. (1995). The art of case study research. Sage.

Sugai, G., \& Horner, R. (1994). Including students with severe behavior problems in general education settings: Assumptions, challenges, and solutions. The Oregon conference monograph, 6, 102-120. 
Sugai, G., Horner, R. H., Dunlap, G., Hieneman, M., Lewis, T. J., Nelson, C. M., \& Turnbull, H. R. (2000). Applying positive behavior support and functional behavioral assessment in schools. Journal of Positive Behavior Interventions, 2(3), 131-143.

Sugai, G., \& Horner, R. (2002). The evolution of discipline practices: School-wide positive behavior supports. Child \& Family Behavior Therapy, 24(1-2), 23-50.

Sugai, G., \& Horner, R. H. (2008). What we know and need to know about preventing problem behavior in schools. Exceptionality, 16(2), 67-77.

Sugai, G., \& Horner, R. H. (2009). Responsiveness-to-intervention and school-wide positive behavior supports: Integration of multi-tiered approaches. Exceptionality, $17,223-237$.

Sugai, G., Horner, R. H., Algozzine, R., Barrett, S., Lewis, T., Anderson, C., \& Simonsen, B. (2010). School-wide positive behavior support: Implementers' blueprint and self-assessment. Eugene, OR: University of Oregon.

Sugai, G., Horner, R. H., Fixsen, D., \& Blase, K. (2010). Developing systems-level capacity for rti implementation: Current efforts and future directions. Response to Intervention: Empowering All Students to Learn-A Critical Account of the Science and Practice, 286-309.

Sugai, G., O'Keeffe, B. V., \& Fallon, L. M. (2011). A contextual consideration of culture and schoolwide positive behavior support. Journal of Positive Behavior Interventions, 1098300711426334. 
Thomas, J. Y., \& Brady, K. P. (2005). The Elementary and secondary education act at 40: Equity, accountability, and the evolving federal role in public education. Review of Research in Education, 51-67.

Thompson, M. L. (1963). Identifying anxieties experienced by student teachers. Journal of Teacher Education, 14(4), 435-439.

Tillery, A. D., Varjas, K., Meyers, J., \& Collins, A. S. (2010). General education teachers' perceptions of behavior management and intervention strategies. Journal of Positive Behavior Interventions, 12(2), 86-102.

Travers, R. M., Rabinowitz, W., \& Nemovicher, E. (1952). The anxieties of a group of student teachers. Educational Administration and Supervision, 38(6), 368-375.

Turner III, D. W. (2010). Qualitative interview design: A practical guide for novice investigators. The Qualitative Report, 15(3), 754.

Wacquant, L. (2001). Deadly symbiosis when ghetto and prison meet and mesh. Punishment \& Society, 3(1), 95-133.

Wald, J., \& Losen, D. J. (2003). Defining and redirecting a school-to-prison pipeline. New Directions for Youth Development, 2003(99), 9-15.

Wang, M. C., Haertel, G. D., \& Walberg, H. J. (1998). Building educational resilience. Fastback 430. Phi Delta Kappa International.

Warren, J. S., Edmonson, H. M., Griggs, P., Lassen, S. R., McCart, A., Turnbull, A., \& Sailor, W. (2003). Urban applications of school-wide positive behavior support critical issues and lessons learned. Journal of Positive Behavior Interventions, 5(2), 80-91. 
Warren, J. S., Bohanon-Edmonson, H. M., Turnbull, A. P., Sailor, W., Wickham, D., Griggs, P., \& Beech, S. E. (2006). School-wide positive behavior support: Addressing behavior problems that impede student learning. Educational psychology review, 18(2), 187-198.

Wendel, F. C., Hoke, F. A., \& Joekel, R. G. (1994). The search for success. Middle School Journal, 25(3), 48-50.

Williams, L. J., \& Downing, J. E. (1998). Membership and belonging in inclusive classrooms: What do middle school students have to say? Research and Practice for Persons with Severe Disabilities, 23(2), 98-110.

Yazan, B. (2015). Three approaches to case study methods in education: Yin, Merriam, and Stake. The Qualitative Report, 20(2), 134-152.

Yin, R. K. (2014). Case study research: Design and methods. (5th ed.). Los Angeles: Sage.

York, L. (1968). Relationships between problems of beginning elementary teachers, their personal characteristics and their preferences for in-service education. Retrieved from ProQuest Information \& Learning.

Zehr, H. (1990). Changing lenses: A new focus for crime and justice. Herald press.

Zhang, A., Musu-Gillette, L., \& Oudekerk, B.A. (2016). Indicators of School Crime and Safety: 2015. National Center for Education Statistics. 


\author{
APPENDIX A \\ Interview Protocol - Teachers
}

\title{
Student Behavior
}

1. What are some things that cause students to exhibit positive behavior in school?

2. What are some things that cause the students to develop negative behavior in school?

3. How do you support positive behavior in the classroom?

4. How to you interrupt negative behavior in the classroom?

5. How do you prevent negative behavior in the classroom?

6. Tell me about your classroom management system.

7. Give me an example of how you've supported positive behavior, interrupted negative behavior and prevented negative behavior in the classroom.

\section{PBIS Training and Implementation}

8. What type of training have you received in Positive Behavior Interventions and Supports (PBIS)?

9. How would you describe Positive Behavior Interventions and Supports (PBIS)?

10. What are some ways PBIS works to prevent negative student behaviors in your classroom? School?

11. What are some ways PBIS doesn't work to prevent negative student behaviors in your classroom? School?

12. Describe the degree of PBIS implementation in your school. 


\section{PBIS Effectiveness and Sustainability}

13. How do you think inappropriate student behaviors in your school have changed as a result of the school-wide implementation of PBIS?

14. What do you think would improve the effectiveness and sustainability of your schools' PBIS?

15. How do you think your schools' PBIS has influenced the overall school climate? Administrator and District Support for PBIS

16. Describe the degree of active support you have received from administration, staff and the school district to support the full implementation of PBIS in your school.

17. What additional support do you need to successfully implement PBIS in your classroom and school to prevent negative student behaviors that prevent teacher instruction?

\section{Teacher Concerns}

18. How do you think teachers feel about your schools' PBIS?

a. How do you think principals/administrators feel about your schools' PBIS?

b. How do you feel about your schools' PBIS?

19. When you think about Schoolwide Positive Behavior Interventions and Supports, what concerns do you have?

20. What is needed to make the behavior and discipline practices in this school work better? 


\section{APPENDIX B \\ Interview Protocol- Principals}

\section{Student Behavior}

1. What are some things that cause students to exhibit positive behaviors in school?

2. What are some things that cause students to develop negative behaviors in school?

3. How do you support positive behavior in your school?

4. How to you interrupt negative behavior in your school?

5. How do you prevent negative behavior in your school?

6. Tell me about your schoolwide behavior system.

7. Give me an example of how you've supported positive behavior, interrupted negative behavior and prevented negative behavior in your school.

\section{PBIS Training and Implementation}

8. What type of training have you received in Positive Behavior Interventions and Supports (PBIS)?

9. How would you describe Positive Behavior Interventions and Supports (PBIS)?

10. What are some ways PBIS works to prevent negative student behaviors in your School?

11. What are some ways PBIS doesn't work to prevent negative student behaviors in School?

12. Describe the degree of PBIS implementation in your school. 


\section{District and Staff Support for PBIS}

13. Describe the degree of active support you have received from the school district and your staff to support the full implementation of PBIS in your school.

14. What additional support do you need to successfully implement PBIS in your school to prevent negative student behaviors that prevent teacher instruction?

\section{PBIS Effectiveness and Sustainability}

15. How do you think inappropriate student behaviors in your school have changed as a result of the school-wide implementation of PBIS?

16. What do you think would improve the effectiveness and sustainability of your schools' PBIS?

17. How do you think your schools' PBIS has influenced the overall school climate?

\section{Teacher \& Principal Concerns}

18. How do you think teachers feel about your schools' PBIS?

a. How do you think principals/administrators feel about your schools' PBIS?

b. How do you feel about your schools' PBIS?

19. When you think about Schoolwide Positive Behavior Interventions and Supports, what concerns do you have?

20. What is needed to make the behavior and discipline practices in this school work better? 


\section{APPENDIX C}

Dorothy Height Elementary School Behavior Intensity Chart

\begin{tabular}{|l|l|}
\hline $\begin{array}{l}\text { Intensity Level 1: Behavior is confined to the observed student: } \\
\text { Talking out, non-compliance, talking/calling out loudly during } \\
\text { instruction, singing during independent work time, out of seat/area, } \\
\text { name calling/arguing, tapping on desk, dis-engaged/drawing during } \\
\text { work time, refusal to work/follow directions, refusal to take } \\
\text { assessment, profanity, eating food in class, talking back to an adult. }\end{array}$ & $\begin{array}{l}\text { No CRT Call } \\
\text { Needed }\end{array}$ \\
\hline $\begin{array}{l}\text { Intensity Level 2: Behavior disrupts others in the student's } \\
\text { immediate area: Hitting/Horseplay, kicking/pushing chairs over, }\end{array}$ & $\begin{array}{l}\text { No CRT Call } \\
\text { Needed }\end{array}$ \\
\hline $\begin{array}{l}\text { Yells "No" or "You can't make me" when given directions, student } \\
\text { cusses directly at another student, uncontrollable crying, uses cell } \\
\text { phone without permission, uses school technology to access website } \\
\text { not school appropriate, excessive noise, uses classroom phone } \\
\text { without permission. }\end{array}$ & \\
\hline $\begin{array}{l}\text { Intensity Level 3: Disrupts everyone in the class: Hitting with the } \\
\text { intent to harm, aggressive horseplay, destroying school property, } \\
\text { stealing, running in the hallways from adults, slams doors repeatedly } \\
\text { or with severe aggression, kicks doors, student leaves the classroom } \\
\text { without permission. }\end{array}$ & $\begin{array}{l}\text { 3 Classroom } \\
\text { Interventions, Call CRT }\end{array}$ \\
\hline $\begin{array}{l}\text { Intensity Level 4: Behavior disrupts other classrooms or common } \\
\text { area of the school: Throwing object with the intent to harm, fighting, } \\
\text { and actually causing harm to another. }\end{array}$ & CRT Call \\
\hline $\begin{array}{l}\text { Intensity Level 5: Behavior Threatens or causes major or legitimate } \\
\text { physical injury to student, staff or others: Evacuation of classroom, } \\
\text { student leaves building/playground/school grounds, student slams } \\
\text { doors that cause injury to others, spitting on others, has } \\
\text { weapons/look-alike drugs, calls 911 falsely, pulls fire alarm. }\end{array}$ & CRT Call \\
\hline
\end{tabular}




\section{CURRICULUM VITA}

NAME: $\quad$ Cassaundra Lynn Watkins

ADDRESS: COLLEGE OF ED. AND HUMAN DEVELOPMENT

UNIVERSITY OF LOUISVILLE

2301 S. Third Street Louisville, KY 40292

cassaundra.watkins@education.ky.gov

\section{EDUCATION:}

University of Louisville, Louisville, KY

Ed. D. in Educational Leadership \& Organizational Development

Dissertation Title: A Case Study of Teacher and Principal Perceptions

Of a School-Wide PBIS Framework in an Urban Elementary School

Dissertation Chairs: Drs. Meera Alagaraja and Blake Haselton

University of Louisville, Louisville, KY

Ed. S., Specialist in Education, Educational Leadership

Area of Concentration: Principalship

University of Louisville, Louisville, KY

M.A.T., Master of Arts in Teaching

Area of Concentration: Early Elementary Education

University of Louisville, Louisville, KY

B.S. in Justice Administration

Area of Concentration: Criminal Justice

University of Louisville, Louisville, KY

A.A. in Paralegal Studies

1992

AWARDS:

Dean's Scholar, University of Louisville

Fall 1998 


\section{TEACHING EXPERIENCE}

Kentucky Department of Education

Educational Recovery Leader/Specialist

2015-

Assist teachers/principals in low-performing schools to

implement systems of continuous improvement that

result in increased student achievement.

Jefferson County Public Schools

Goal Clarity Coach

$2012-2015$

Facilitated PLCs, professional development trainings,

analyzed assessment data, completed School

Improvement Plan and facilitated teacher modeling and

instructional support.

Jefferson County Public Schools

Teacher

2007-2012

Delivered rigorous, differentiated, standards based lessons.

Assessed student learning and analyzed results for RTI/Enrichment

\section{RELATED EXPERIENCE}

University of Louisville, Louisville, KY

Doctoral Candidate

Feb. 2016

Educational Leadership and Organizational Development

Jefferson County Public Schools, Louisville, KY

Substitute Teacher

2001-2004

Instruct elementary, middle and high school students.

\section{LANGUAGES}

English- native language

\section{MEMBERSHIPS}

The Institute for Performance Improvement (TIFPI) -

Certified School Improvement Specialist 